\title{
Statistics of eigenvalue dispersion indices: quantifying the magnitude of
}

\section{phenotypic integration}

Version 2 for bioRxiv (2021.08.07)

Junya Watanabe

Department of Earth Sciences, University of Cambridge, Downing Street, Cambridge, CB2

3EQ, United Kingdom

jw2098@cam.ac.uk

https://orcid.org/0000-0002-9810-5286 


\section{Abstract}

2 Quantification of the magnitude of trait covariation plays a pivotal role in the study of

3 phenotypic evolution, for which statistics based on dispersion of eigenvalues of a covariance

4 or correlation matrix — eigenvalue dispersion indices—are commonly used. This study

5 remedies major issues over the use of these statistics, namely, a lack of clear understandings

6 on their statistical justifications and sampling properties. The relative eigenvalue variance of

7 a covariance matrix is known in the statistical literature a test statistic for sphericity, thus is

8 an appropriate measure of eccentricity of variation. The same of a correlation matrix is equal

9 to the average squared correlation, which has a straightforward interpretation as a measure of

10 integration. Expressions for the mean and variance of these statistics are analytically derived

11 under multivariate normality, clarifying the effects of sample size $N$, number of variables $p$,

12 and parameters on sampling bias and error. Simulations confirmed that approximations

13 involved are reasonably accurate with a moderate sample size $(N \geq 16-64)$. Importantly,

14 sampling properties of these indices are not adversely affected by a high $p: N$ ratio, promising

15 their utility in high-dimensional phenotypic analyses. They can furthermore be applied to

16 shape variables and phylogenetically structured data with appropriate modifications.

18 Keywords: covariance matrix; evolutionary constraint; morphometrics; phenotypic

19 integration; quantitative genetics; Wishart distribution.

\section{Introduction}

22 Analysis of trait covariation plays a central role in investigations into evolution of

23 quantitative traits. The well-known quantitative genetic theory of correlated traits predicts

24 that evolutionary response in a population under selection is dictated by the additive genetic

25 covariance matrix $\mathbf{G}$ as well as the selection gradient (Lande, 1979; Lande \& Arnold, 1983). 
26 Short-term evolutionary changes of a population are expected to be concentrated in major

27 axes of the $\mathbf{G}$ matrix (Schluter, 1996). Arguably, the structure of the $\mathbf{G}$ matrix can be

28 approximated by that of the phenotypic covariance matrix for certain types of traits

29 (Cheverud, 1988, 1996; Roff, 1995; Dochtermann, 2011; Sodini et al., 2018), so the latter

30 could be analyzed when accurate estimation of the $\mathbf{G}$ matrix is not feasible. These theories

31 and conjectures spurred extensive theoretical and empirical explorations on character

32 covariation as an evolutionary constraint (e.g., Steppan et al., 2002; Chenoweth et al., 2010;

33 Pitchers et al., 2014; Hansen et al., 2019 and references therein). Partly fueled by these

34 developments, the study of phenotypic integration has developed as an active field of

35 research, where various aspects of character covariation are investigated with diverse

36 motivations and scopes (e.g., Olson \& Miller, 1958; Cheverud, 1982; Goswami, 2006;

37 Hallgrímsson et al., 2009; Armbruster et al., 2014; Felice et al., 2018). In the latter context,

38 many different levels of organismal variation can be subjects of research, such as static,

39 ontogenetic, and evolutionary levels (Klingenberg, 2014). For example, relationships

40 between within-population integration and evolutionary rate and/or trajectories have attained

41 much attention as potential links between micro- and macroevolutionary phenomena (e.g.,

42 Klingenberg et al., 2012; Renaud \& Auffray, 2013; Bolstad et al., 2014; Goswami et al.,

43 2015; Haber, 2015, 2016).

44 An obvious target of investigation in these contexts is quantitative analysis of the

45 magnitude of constraint or integration entailed in covariance structures. In particular, this

46 paper concerns the methodology for quantifying the overall magnitude of covariation within a

47 set of traits. Quantification of relative (in)dependence between multiple sets of traits- the

48 modularity-integration spectrum - is another major way of studying integration which has

49 separate methodological frameworks (e.g., Goswami \& Polly, 2010; Adams, 2016; Goswami

50 \& Finarelli, 2016; Adams \& Collyer, 2019a). Demonstrating the presence of integration with 
51 a statistically justified measure can be the scope of an empirical analysis, sometimes as a part

52 of testing combined hypotheses (e.g., Brommer, 2014; Watanabe, 2018). A univariate

53 summary statistic for magnitude of integration can conveniently be used in comparative

54 analyses across developmental stages, populations, or phylogeny (e.g., Marroig et al., 2009;

55 Porto et al., 2009; Haber, 2016). A plethora of statistics have been proposed for such

56 purposes from various standpoints (e.g., Van Valen, 1974, 2005; Cheverud et al., 1983, 1989;

57 Wagner, 1984; Cane, 1993; Hansen \& Houle, 2008; Agrawal \& Stinchcombe, 2009;

58 Kirkpatrick, 2009; Pavlicev et al., 2009; Armbruster et al., 2009, 2014; Haber, 2011). One of

59 the most popular classes of such statistics is based on the dispersion of eigenvalues of a

60 covariance or correlation matrix. These statistics have the forms

$$
\begin{gathered}
V=\frac{1}{p} \sum_{i=1}^{p}\left(\lambda_{i}-\bar{\lambda}\right)^{2}, \\
V_{\mathrm{rel}}=\frac{\sum_{i=1}^{p}\left(\lambda_{i}-\bar{\lambda}\right)^{2}}{p(p-1) \bar{\lambda}^{2}},
\end{gathered}
$$

61 where $p$ is the number of variables (traits), $\lambda_{i}$ is the $i$ th eigenvalue of the covariance or

62 correlation matrix under analysis, and $\bar{\lambda}$ is the average of the eigenvalues. Here, $V$ is the most

63 naïve form of eigenvalue dispersion, and $V_{\text {rel }}$ is a scaled version which ranges between 0 and

64 1. Formal definitions are given below with distinction between population and sample

65 quantities. Some authors use square root or a constant multiple of these forms, but such

66 variants essentially bear identical information when calculated from the same matrix.

67 Alternative terms for this class of statistics include the tightness (for $V_{\text {rel }}$; Van Valen, 1974;

68 later used for $\sqrt{V_{\text {rel }}}$ by Van Valen, 2005), integration coefficient of variation (for

$69 \sqrt{(p-1) V_{\text {rel }}}$; Shirai \& Marroig, 2010), and phenotypic integration index (for $V$; Torices \&

70 Muñoz-Pajares, 2015). In this paper, $V$ and $V_{\text {rel }}$ are called the eigenvalue variance and

71 relative eigenvalue variance, respectively, to take a balance between brevity and 
72 descriptiveness. These quantities are not to be confused with the sampling variance

73 associated with eigenvalues in a sample (see below).

74 Since eigenvalues of a covariance or correlation matrix correspond to the variance

75 along the corresponding eigenvectors (principal components), these statistics are supposed to

76 represent eccentricity of variation across directions in a trait space (Fig. 1; Wagner, 1984).

77 Cheverud et al. (1983) and Wagner (1984) were the first to propose using $V$ of a correlation

78 matrix for quantifying magnitude of integration. Pavlicev et al. (2009) devised $V_{\text {rel }}$ for a

79 correlation matrix, and explored its relationships to correlation structures in certain

80 biologically relevant conditions. Haber (2011) pointed out similarity between these indices

81 and Van Valen's (1974) tightness index for a covariance matrix, and proposed that these

82 indices can be applied to either covariance or correlation matrices with slightly different

83 interpretations. Eigenvalue dispersion indices are frequently used in empirical analyses of

84 phenotypic integration at various levels of organismal variation, from phenotypic covariance

85 at the population level to evolutionary covariance at the interspecific level (e.g., Ordano et al.,

86 2008; Torices \& Mendez, 2014; Haber, 2016; Haber \& Dworkin, 2017; Watanabe, 2018;

87 Arlegi et al., 2020). However, use of these indices has been criticized for a lack of clear

88 statistical justifications; it has not been known — or not widely appreciated by biologists—

89 exactly what they are designed to measure, beyond the intuitive allusion to eccentricity

90 mentioned above (Hansen \& Houle, 2008; Blows \& McGuigan, 2015; Hansen et al., 2019).

91 Another fundamental issue over the eigenvalue dispersion indices is a virtual lack of

92 systematic understanding of their sampling properties. In empirical analyses, eigenvalue

93 dispersion indices are calculated from sample covariance or correlation matrices, but interests

94 will be in making inferences for the underlying populations. For example, interest may be in

95 detecting the presence of constraint in a population, i.e., testing the null hypothesis of

96 sphericity. As detailed below, however, sample eigenvalues are always estimated with error, 
so that $V$ and $V_{\text {rel }}$ calculated from them take a positive value even if the corresponding

population values are 0 . In other words, empirical eigenvalue dispersion indices are biased e.g., expectation and variance. in the literature (Wagner, 1984; Cheverud et al., 1989; Grabowski \& Porto, 2017; see also Marroig et al., 2012; Björklund, 2019). Simulation-based studies have been undertaken to sketch sampling distributions of eigenvalue dispersion indices and related statistics (Haber, 2011; Grabowski \& Porto, 2017; Machado et al., 2019; Jung et al. 2020). However, these approaches hardly give any systematic insight beyond the specific conditions considered.

108 Analytic results should preferably be sought to comprehend the sampling bias and error. In 109 this regard, it is notable that Wagner (1984) derived the first two moments of eigenvalues of 110 sample covariance and correlation matrices under the null conditions. The variance of sample

111 eigenvalues obtained from these moments has later been used as an estimate of sampling bias

112 in these conditions (e.g., Cheverud et al., 1989). Strictly speaking, however, the variance of a 113 sample eigenvalue is fundamentally different from the expectation of the eigenvalue variance

$114 V$. These quantities are identical for correlation matrices under the null hypothesis, but this is

115 not the case for covariance matrices where the covariances between sample eigenvalues

116 cannot be ignored (see below). Furthermore, Wagner's (1984) results have a few restrictive

117 conditions: variables to have the means of 0 , or equivalently, to be centered at the population

118 mean rather than the sample mean as is done in most empirical analyses (although this was

119 probably appropriate in the strict context of his theoretical model); and the sample size $N$ to

120 be equal to or larger than the number of variables $p$, so their applicability to $p>N$ conditions

121 has not been demonstrated. 
122 In addition to the naïve null condition of no integration, moments under arbitrary

123 conditions are also desired. Such would be useful in testing hypotheses about the magnitude

124 (rather than the mere presence/absence) of integration (Harder, 2009; Fornoni et al., 2009)

125 and comparing the magnitudes between different samples (Cheverud et al., 1989). Also, the

126 assumption of no covariation is intrinsically inappropriate as a null hypothesis for shape

127 variables where raw data are transformed in such a way that individual "variables" are

128 necessarily dependent on one another (e.g., Mitteroecker et al., 2012). For this type of data, a

129 covariance matrix with an appropriate structure needs to be specified as the null model

130 representing the intrinsic covariation.

131 This paper addresses the issues over the eigenvalue dispersion indices mentioned

132 above. It first gives a theoretical overview of these statistics to clarify their statistical

133 justifications, particularly in connection to the sphericity test in multivariate analysis. Then

134 exact and approximate expressions are analytically derived for the expectation and variance

135 of $V$ and $V_{\text {rel }}$ of sample covariance and correlation matrices under the null and arbitrary

136 conditions, assuming the multivariate normality of variables. These expressions are derived

137 without any assumption on $p$ or $N$, except for the variance of $V$ and $V_{\text {rel }}$ of a correlation

138 matrix under arbitrary conditions, which is based on large-sample asymptotic theories.

139 Simulations were subsequently conducted to obtain systematic insights into sampling

140 properties and to evaluate the accuracy of the approximate expressions. Extensions into shape

141 variables and phylogenetically structured data are briefly discussed.

\section{Theory}

\section{Preliminaries}

145 For the purpose here, the distinction between population and sample quantities is essential.

146 Corresponding Greek and Latin letters are used as symbols for the former and latter, 
147 respectively. Let $\boldsymbol{\Sigma}$ be the $p \times p$ population covariance matrix, whose $(i, j)$-th element $\sigma_{i j}$ is

148 the population variance $(i=j)$ or covariance $(i \neq j)$. It is a symmetric, nonnegative definite

149 matrix with the eigendecomposition

$$
\boldsymbol{\Sigma}=\mathbf{Y} \boldsymbol{\Lambda} \mathbf{Y}^{T}
$$

151 where the superscript ${ }^{T}$ denotes matrix transposition, $\mathbf{Y}$ is an orthogonal matrix of

152 eigenvectors $\left(\mathbf{Y} \mathbf{Y}^{T}=\mathbf{Y}^{T} \mathbf{Y}=\mathbf{I}_{p}\right.$ where $\mathbf{I}_{p}$ is the $p \times p$ identity matrix $)$, and $\boldsymbol{\Lambda}$ is a diagonal

153 matrix whose diagonal elements are the eigenvalues $\lambda_{1}, \lambda_{2}, \ldots, \lambda_{p}$ of $\Sigma$ (population

154 eigenvalues). For convenience, the eigenvalues are arranged in the non-increasing order:

$155 \lambda_{1} \geq \lambda_{2} \geq \cdots \geq \lambda_{p} \geq 0$. Let $\boldsymbol{\mu}$ be the $p \times 1$ population mean vector.

$156 \quad$ Let $\mathbf{X}$ be an $N \times p$ observation matrix consisting of $p$-variate observations, which are

157 individually denoted as $\mathbf{x}_{i}(p \times 1$ vector; transposed in the rows of $\mathbf{X})$. (No strict notational

158 distinction is made between a random variable and its realization.) At this point, $N$

159 observations are assumed to be identically and independently distributed (i.i.d.). The sample

160 covariance matrix $\mathbf{S}$ and cross-product matrix $\mathbf{A}$ are defined as

$$
\mathbf{S}=\frac{1}{n_{*}} \mathbf{A}=\frac{1}{n_{*}}\left(\mathbf{X}-\mathbf{1}_{N} \overline{\mathbf{x}}^{T}\right)^{T}\left(\mathbf{X}-\mathbf{1}_{N} \overline{\mathbf{x}}^{T}\right),
$$

162 where $\mathbf{1}_{N}$ is a $N \times 1$ column vector of 1 's, $\overline{\mathbf{x}}=\sum_{i=1}^{N} \mathbf{x}_{i} / N$ is the sample mean vector, and $n_{*}$

163 denotes an appropriate devisor; e.g., $n_{*}=N-1$ for the ordinary unbiased estimator, and

$164 n_{*}=N$ for the maximum likelihood estimator under the normal distribution. The $(i, j)$-th

165 element of $\mathbf{S}$, denoted $s_{i j}$, is the sample variance or covariance. The eigendecomposition of $\mathbf{S}$

166 is constructed in the same way as above:

$$
\mathbf{S}=\mathbf{U} \mathbf{L} \mathbf{U}^{T},
$$

where $\mathbf{U}$ is an orthogonal matrix of sample eigenvectors and $\mathbf{L}$ is a diagonal matrix whose

169 elements are the sample eigenvalues $l_{1}, l_{2}, \ldots, l_{p}$. 
170 In what follows, the following identity entailed by the orthogonality of $\mathbf{U}$ is frequently

171

172

173

174

175

176

177

178

180

utilized:

$$
\sum_{i=1}^{p} s_{i i}^{r}=\operatorname{tr}\left(\mathbf{S}^{r}\right)=\operatorname{tr}\left(\mathbf{U L U} \mathbf{U}^{T} \mathbf{U L U} \mathbf{U}^{T} \ldots \mathbf{U L U} \mathbf{U}^{T}\right)=\operatorname{tr}\left(\mathbf{L}^{r}\right)=\sum_{i=1}^{p} l_{i}^{r}, r=1,2, \ldots
$$

where $\operatorname{tr}(\cdot)$ denotes the matrix trace operator, i.e., summation of the diagonal elements; the parentheses are omitted for visual clarity when little ambiguity exists. The sum of variances $\operatorname{tr} \mathbf{S}=\operatorname{tr} \mathbf{L}$ is called total variance. Note that equation 4 holds even when $N-1<p$, in which case $l_{i}=0$ for some $i$. In other words, when $N-1<p$, the sample total variance is in a way concentrated in a subspace with fewer dimensions than the full space.

The population and sample correlation matrices $\mathbf{P}$ and $\mathbf{R}$, whose $(i, j)$-th elements are the population and sample correlation coefficients $\rho_{i j}$ and $r_{i j}$, respectively, are obtained by standardizing $\boldsymbol{\Sigma}$ and $\mathbf{S}$ :

$$
\begin{aligned}
& \mathbf{P}=\operatorname{diag}\left(\sigma_{i i}^{-1 / 2}\right) \boldsymbol{\Sigma} \operatorname{diag}\left(\sigma_{i i}^{-1 / 2}\right), \\
& \mathbf{R}=\operatorname{diag}\left(s_{i i}^{-1 / 2}\right) \boldsymbol{S} \operatorname{diag}\left(s_{i i}^{-1 / 2}\right),
\end{aligned}
$$

where $\operatorname{diag}(\cdot)$ stands for the $p \times p$ diagonal matrix with the designated $i$ th elements. Their eigendecomposition is defined as for covariance matrices, and the eigenvalues are denoted with the same symbols. For any $i, \rho_{i i}=r_{i i}=1$, and hence, for correlation matrices

$$
\operatorname{tr} \mathbf{P}=\operatorname{tr} \boldsymbol{\Lambda}=\operatorname{tr} \mathbf{R}=\operatorname{tr} \mathbf{L}=p .
$$

In what follows, the notations $\mathrm{E}(\cdot), \operatorname{Var}(\cdot)$, and $\operatorname{Cov}(\cdot, \cdot)$ are used for the expectation (mean), variance, and covariance of random variables, respectively.

\section{Eigenvalue dispersion}

The eigenvalue variance $V$ is defined as:

$$
\begin{aligned}
& V(\boldsymbol{\Sigma})=\frac{1}{p} \sum_{i=1}^{p}\left(\lambda_{i}-\bar{\lambda}\right)^{2}, \\
& V(\mathbf{S})=\frac{1}{p} \sum_{i=1}^{p}\left(l_{i}-\bar{l}\right)^{2}
\end{aligned}
$$


where $\bar{\lambda}$ and $\bar{l}$ are the averages of the population and sample eigenvalues, respectively

$\left(\bar{\lambda}=\sum_{i=1}^{p} \lambda_{i} / p=\operatorname{tr} \boldsymbol{\Lambda} / p, \bar{l}=\sum_{i=1}^{p} l_{i} / p=\operatorname{tr} \mathbf{L} / p\right)$. Note that $V(\boldsymbol{\Sigma})$ is a quantity pertaining to

196 the population, whereas $V(\mathbf{S})$ is a sample statistic. The definition here follows the convention

197 in the literature that $p$, rather than $p-1$, is used as the divisor (e.g., Cheverud et al., 1983;

198 Pavlicev et al., 2009; Haber, 2011). The latter might be more suitable for $V(\mathbf{S})$ because the

199 sum of squares is taken around the average sample eigenvalue which is a random variable.

200 After all, however, the choice of $p-1$ is not so useful because $V(\mathbf{S})$ cannot be an unbiased

201 estimator of $V(\boldsymbol{\Sigma})$ even with that choice (below).

Note that the average and sum of squares are taken across all $p$ eigenvalues, even if

210 On the other hand, for a fixed $\bar{\lambda}$, it takes a single maximum of $(p-1) \bar{\lambda}^{2}$ at $(p \bar{\lambda}, 0, \ldots, 0)$ (e.g.,

211 Van Valen, 1974; Machado et al., 2019). Hence, not only is $V(\boldsymbol{\Sigma})$ scale-variant, but also its

212 range depends on $p-1$. Therefore, it is often useful to standardize $V$ by division with this

213 maximum to obtain the relative eigenvalue variance $V_{\text {rel }}$ :

$$
\begin{aligned}
& V_{\text {rel }}(\boldsymbol{\Sigma})=\frac{\sum_{i=1}^{p}\left(\lambda_{i}-\bar{\lambda}\right)^{2}}{p(p-1) \bar{\lambda}^{2}}, \\
& V_{\text {rel }}(\mathbf{S})=\frac{\sum_{i=1}^{p}\left(l_{i}-\bar{l}\right)^{2}}{p(p-1) \bar{l}^{2}} .
\end{aligned}
$$

216 Because of the standardization, $V_{\text {rel }}$ ranges between 0 and 1 . This is a heuristic introduction

217 of $V_{\text {rel }}$ from $V$, but it will be seen below that $V_{\text {rel }}(\mathbf{S})$ has a clearer theoretical justification. 
219 6), these are

$$
\begin{gathered}
V(\mathbf{P})=\frac{1}{p} \sum_{i=1}^{p}\left(\lambda_{i}-1\right)^{2}, \\
V(\mathbf{R})=\frac{1}{p} \sum_{i=1}^{p}\left(l_{i}-1\right)^{2} . \\
V_{\text {rel }}(\mathbf{P})=\frac{\sum_{i=1}^{p}\left(\lambda_{i}-1\right)^{2}}{p(p-1)} \\
V_{\text {rel }}(\mathbf{R})=\frac{\sum_{i=1}^{p}\left(l_{i}-1\right)^{2}}{p(p-1)} .
\end{gathered}
$$

224 In the following discussions, we will concentrate on $V_{\text {rel }}$ for correlation matrices, because

$V(\mathbf{R})$ and $V_{\text {rel }}(\mathbf{R})$ are proportional to each other by the factor $p-1$, and hence their

226 distributions are identical up to this scaling. This is in contrast to those of covariance matrices,

227 where $\bar{l}$ in the denominator in $V_{\text {rel }}(\mathbf{S})$ is a random variable and affects sampling properties.

228 Importantly, a single value of $V_{\text {rel }}$ in general corresponds to multiple combinations of

229 eigenvalues even if the average eigenvalue is fixed, except when $p=2$ or under the extreme

230 conditions $V_{\text {rel }}=0$ and $V_{\text {rel }}=1$ (Fig. 1). As such, it is not always straightforward to discern

231 how intermediate values of $V_{\text {rel }}$ are translated into actual covariance structures when $p>2$.

232 Nevertheless, it is possible to show that $V_{\text {rel }}>0.5$ cannot happen when multiple leading

233 eigenvalues are of the same magnitude (Appendix A); in other words, such a large value

234 indicates dominance of the first principal component.

235 As would be obvious from the definition, $V$ and $V_{\text {rel }}$ of covariance matrices only

236 describe the (relative) magnitudes of eigenvalues - proportions of the axes of variation-and

237 do not reflect any information of eigenvectors-directions of the axes. A large eigenvalue of

238 a covariance matrix can represent, e.g., strong covariation between equally varying traits or

239 large variation of a single trait uncorrelated with others; either of these cases describes

240 eccentricity of variation in the multivariate space. By contrast, a large eigenvalue of a

241 correlation matrix can only happen in the presence of correlation. Therefore, a large 
242 eigenvalue dispersion in a correlation matrix constrains conformation of eigenvectors to a

243 certain extent. The correlations can nevertheless be realized in various ways depending on

244 eigenvectors, whose conformation does influence the sampling distribution of $V_{\text {rel }}(\mathbf{R})$ (see

245 below).

246 For covariance matrices, $V_{\text {rel }}(\mathbf{S})$ has a natural relation to the test of sphericity, i.e., test

247 of the null hypothesis that $\boldsymbol{\Sigma}=\sigma^{2} \mathbf{I}_{p}$ for an arbitrary positive constant $\sigma^{2}$. Simple

248 transformations from equation 8 lead to

$$
V_{\mathrm{rel}}(\mathbf{S})=\frac{1}{p-1}\left(p \frac{\sum l_{i}^{2}}{\left(\sum l_{i}\right)^{2}}-1\right)
$$

250 By noting $\sum l_{i}^{2} /\left(\sum l_{i}\right)^{2}=\operatorname{tr}\left(\mathbf{S}^{2}\right) /(\operatorname{tr} \mathbf{S})^{2}=\operatorname{tr}\left(\mathbf{A}^{2}\right) /(\operatorname{tr} \mathbf{A})^{2}$ (see eqs. 2 and 4$), V_{\text {rel }}(\mathbf{S})$ in the

251 form of equation 10 is exactly John's (1972) $T$ statistic for the test of sphericity (see also

252 Ledoit \& Wolf, 2002). Beyond the intuition that it measures eccentricity of variation along

253 principal components, this statistic (and its linear functions) can be justified as the most

254 powerful test statistic in the proximity of the null hypothesis under multivariate normality,

255 among the class of such statistics that are invariant against translation by a constant vector,

256 uniform scaling, and orthogonal rotation (John, 1971, 1972; Sugiura, 1972; Nagao, 1973). On

257 the other hand, $V(\mathbf{S})$ does not seem to have as much theoretical justification, but rather has a

258 practical advantage in the tractability of its moments and ease of correcting sampling bias

259 (see below).

260 For a correlation matrix, $V_{\text {rel }}$ is a measure of association between variables. Following

261 similar transformations, it is straightforward to see

$$
\begin{aligned}
V_{\text {rel }}(\mathbf{R}) & =\frac{\operatorname{tr}\left(\mathbf{R}^{2}\right)-p}{p(p-1)} \\
& =\frac{2}{p(p-1)} \sum_{i<j}^{p} r_{i j}^{2},
\end{aligned}
$$


263 because $r_{i i}^{2}=1$ for all $i$. This relationship has been known in the statistical literature (e.g.,

264 Gleason \& Staelin, 1975; Durand \& Le Roux, 2017), and empirically confirmed by Haber

265 (2011). This statistic is used as a measure of overall association between variables (e.g.,

266 Schott, 2005; Durand \& Le Roux, 2017), with the corresponding null hypothesis being

$267 \mathbf{P}=\mathbf{I}_{p}$.

268

269 Sampling properties of eigenvalues

270 The distribution of eigenvalues of $\mathbf{S}$, or equivalently those of $\mathbf{A}$ (which are $n_{*}$ times those of

271 S), has been extensively investigated in the literature of multivariate analysis (see, e.g.,

272 Jolliffe, 2002; Anderson, 2003). Unfortunately, however, most of such results are of limited

273 value for the present purposes. On the one hand, forms of the exact joint distribution of the

274 eigenvalues of $\mathbf{A}$ are known under certain assumptions on the population eigenvalues (e.g.,

275 Muirhead, 1982: pp. 107, 388), but they do not allow for much intuitive interpretation (let

276 alone direct evaluation of moments), apart from the following points: 1) sample eigenvalues

277 are not stochastically independent from one another; and 2) the distribution of sample

278 eigenvalues are only dependent on the population eigenvalues, but not on the population

279 eigenvectors. On the other hand, a substantial body of results is available for large-sample

280 asymptotic distributions of sample eigenvalues (assuming $n \rightarrow \infty, p$ being constant; e.g.,

281 Anderson, 1963, 2003), but their accuracy under finite $n$ conditions is questionable. For

282 example, a well-known asymptotic result states that $l_{i} \sim N\left(\lambda_{i}, 2 \lambda_{i}^{2} / n\right)$ and $\operatorname{Cov}\left(l_{i}, l_{j}\right) \approx 0$ for

$283 i \neq j$, assuming $\lambda_{i} \neq \lambda_{j}$ and $n_{*}=n$ (Girshick, 1939; Anderson, 1963; Srivastava \& Khatri,

284 1979). However, these expressions ignore terms of order higher than $O\left(n^{-1}\right)$ - that is, all

285 terms with $>1$ powers of $n$ in the denominator-whose magnitude can be substantial for finite

$286 n$. Indeed, with further evaluation of higher-order terms, it becomes evident that sample 
eigenvalues are biased estimators of the population equivalents, where large eigenvalues are

288 prone to overestimation and small ones are prone to underestimation, and that $\operatorname{Cov}\left(l_{i}, l_{j}\right)=$

$2 \lambda_{i} \lambda_{j} /\left[\left(\lambda_{i}-\lambda_{j}\right) n\right]^{2}+O\left(n^{-3}\right)$ for $i \neq j$, again assuming $\lambda_{i} \neq \lambda_{j}$ (Lawley, 1956; Srivastava

\& Khatri, 1979). In general, the covariance between sample eigenvalues is nonzero.

dimensional asymptotic conditions (assuming $n \rightarrow \infty, p \rightarrow \infty$, and $p / n$ typically held finite than analytic results. The theories primarily concern limiting distributions of largest sample eigenvalues or the entire bulk of sample eigenvalues, and do not seem directly applicable to the present analysis, which requires accurate expressions of certain higher-order moments of sum of eigenvalues (see below).

Much less is known about eigenvalues of a sample correlation matrix $\mathbf{R}$ (Jolliffe,

2002), whose distribution seems intractable except under certain special conditions

302 (Anderson, 1963). Large-sample asymptotic results indicate that the limiting distribution

$303(n \rightarrow \infty)$ of an eigenvalue of $\mathbf{R}$ is centered around the corresponding population eigenvalue

304 but its variance depends on population eigenvectors (Anderson, 1963; Konishi, 1979), unlike are equivalent to $V(\boldsymbol{\Sigma})=V_{\text {rel }}(\boldsymbol{\Sigma})=0$ and $V_{\text {rel }}(\mathbf{P})=0$, respectively. Even under these

310 conditions, nonzero sampling variance in sample eigenvalues renders $V(\mathbf{S})>0, V_{\text {rel }}(\mathbf{S})>0$,

311 and $V_{\text {rel }}(\mathbf{R})>0$ with probability 1 , because these statistics are calculated from sum of 
312 squares. The primary aim here is to derive explicit expressions for this sampling bias

313 (expectation), as well as sampling variance.

314 It should be remembered that the expectation of the eigenvalue variance $E[V(\mathbf{S})]$ is

315 fundamentally different from the variance of eigenvalues $\operatorname{Var}\left(l_{i}\right)$. This point will be clarified

316 by the following transformation:

$$
\begin{aligned}
\mathrm{E}[V(\mathbf{S})] & =\frac{1}{p} \mathrm{E}\left(\sum_{i=1}^{p} l_{i}^{2}\right)-\frac{1}{p^{2}} \mathrm{E}\left[\left(\sum_{i=1}^{p} l_{i}\right)^{2}\right] \\
& =\frac{p-1}{p^{2}} \sum_{i=1}^{p} \mathrm{E}\left(l_{i}^{2}\right)-\frac{1}{p^{2}} \sum_{i \neq j}^{p}\left[\mathrm{E}\left(l_{i}\right) \mathrm{E}\left(l_{j}\right)+\operatorname{Cov}\left(l_{i}, l_{j}\right)\right] .
\end{aligned}
$$

318 Under the null hypothesis, the moments are equal across all $i$, and the above simplifies into

$$
\frac{p-1}{p}\left[\operatorname{Var}\left(l_{i}\right)-\operatorname{Cov}\left(l_{i}, l_{j}\right)\right], i \neq j
$$

If $\operatorname{Cov}\left(l_{i}, l_{j}\right)$ were zero, the expectation would coincide with $(p-1) \operatorname{Var}\left(l_{i}\right) / p$, which can be

unlike the case for correlation matrices, where $\mathrm{E}[\operatorname{V(R)}]=\operatorname{Var}\left(l_{i}\right)$ holds under the null

324 hypothesis, because $\bar{l}$ is a constant and equals $\mathrm{E}\left(l_{i}\right)=1$.

In the following discussions on moments of eigenvalue dispersion indices, 
332 2003). Therefore, for analyzing statistics associated with sample covariance or correlation

333 matrices, we can conveniently consider

$$
\mathbf{S}=\frac{1}{n_{*}} \mathbf{Z}^{T} \mathbf{Z}
$$

335 without loss of generality, by bearing in mind the distinction between the degree of freedom

$336 n$ and sample size $N$. From elementary moments of the normal distribution, we have

$$
\begin{gathered}
\mathrm{E}\left(s_{i j}\right)=\frac{1}{n_{*}} \sum_{k=1}^{n} \mathrm{E}\left(z_{k i} z_{k j}\right)=\frac{n}{n_{*}} \sigma_{i j}, \\
\mathrm{E}\left(s_{i j} s_{k m}\right)=\frac{n^{2}}{n_{*}^{2}}\left[\sigma_{i j} \sigma_{k m}+\frac{1}{n}\left(\sigma_{i k} \sigma_{j m}+\sigma_{i m} \sigma_{j k}\right)\right],
\end{gathered}
$$

where $z_{i j}, s_{i j}, \sigma_{i j}$ and the like are the $(i, j)$-th elements of $\mathbf{Z}, \mathbf{S}$, and $\boldsymbol{\Sigma}$, respectively.

\section{Moments under null hypotheses}

\section{Covariance matrix}

343 Before proceeding to arbitrary conditions, let us consider the null hypothesis of sphericity:

$344 \boldsymbol{\Sigma}=\sigma^{2} \mathbf{I}_{p}$. For the expectation of $V(\mathbf{S})$, we need $\operatorname{Var}\left(l_{i}\right)$ and $\operatorname{Cov}\left(l_{i}, l_{j}\right)$, or equivalently

$\mathrm{E}\left(\sum_{i=1}^{p} l_{i}^{2}\right)$ and $\mathrm{E}\left[\left(\sum_{i=1}^{p} l_{i}\right)^{2}\right]$ (see eqs. 12 and 13); we will proceed with the latter here. By

346 use of equations 4 and 15 , we have

$$
\begin{aligned}
\mathrm{E}\left(\sum_{i=1}^{p} l_{i}^{2}\right) & =\mathrm{E}\left(\sum_{i, j=1}^{p} s_{i j}^{2}\right) \\
& =\mathrm{E}\left(\sum_{i=1}^{p} s_{i i}^{2}+\sum_{i \neq j}^{p} s_{i j}^{2}\right) \\
& =\left[p \mathrm{E}\left(s_{i i}^{2}\right)+p(p-1) \mathrm{E}\left(s_{i j}^{2}\right)\right] \\
& =\frac{p n}{n_{*}^{2}}(p+n+1) \sigma^{4},
\end{aligned}
$$

348 and similarly 


$$
\begin{aligned}
\mathrm{E}\left[\left(\sum_{i=1}^{p} l_{i}\right)^{2}\right] & =\mathrm{E}\left[\left(\sum_{i=1}^{p} s_{i i}\right)^{2}\right] \\
& =\mathrm{E}\left[\sum_{i=1}^{p} s_{i i}^{2}+\sum_{i \neq j}^{p} s_{i i} s_{j j}\right] \\
& =\left[p \mathrm{E}\left(s_{i i}^{2}\right)+p(p-1) \mathrm{E}\left(s_{i i} s_{j j}\right)\right] \\
& =\frac{p n}{n_{*}^{2}}(p n+2) \sigma^{4} .
\end{aligned}
$$

350 Then, inserting these results into equation 12 ,

$$
\mathrm{E}[V(\mathbf{S})]=\frac{n}{p n_{*}^{2}}(p-1)(p+2) \sigma^{4}
$$

352 Alternatively, it could be seen that $\operatorname{Var}\left(l_{i}\right)=n(p+1) \sigma^{2} / n_{*}^{2}$ and $\operatorname{Cov}\left(l_{i}, l_{j}\right)=-n \sigma^{4} / n_{*}^{2}$

353 for $i \neq j$ (see also Girshick, 1939), with which equation 13 yields the identical result. The variance of $V(\mathbf{S})$ is, by equation 12 ,

$$
\operatorname{Var}[V(\mathbf{S})]=\frac{1}{p^{2}} \operatorname{Var}\left[\sum_{i=1}^{p} l_{i}^{2}\right]+\frac{1}{p^{4}} \operatorname{Var}\left[\left(\sum_{i=1}^{p} l_{i}\right)^{2}\right]-2 \frac{1}{p^{3}} \operatorname{Cov}\left[\sum_{i=1}^{p} l_{i}^{2},\left(\sum_{i=1}^{p} l_{i}\right)^{2}\right]
$$

356 The relevant moments can most conveniently be found as a special case of general

357 expressions under arbitrary $\boldsymbol{\Sigma}$ (see below and Appendix B), although direct derivation using normal moments is possible:

$$
\begin{gathered}
\mathrm{E}\left[\left(\sum l_{i}^{2}\right)^{2}\right]=\frac{p n}{n_{*}^{4}}\left(p^{3} n+p n^{3}+2 p^{2} n^{2}+2 p^{2} n+2 p n^{2}+8 p^{2}+8 n^{2}+21 p n\right. \\
+20 p+20 n+20) \sigma^{8}
\end{gathered}
$$


360 Inserting these into equation 19 yields

361

$$
\operatorname{Var}[V(\mathbf{S})]=\frac{4 n}{p^{3} n_{*}^{4}}(p-1)(p+2)\left(2 p^{2}+p n+3 p-6\right) \sigma^{8}
$$

363 previously been derived by John [1972]). Recalling the form of equation 10,

$$
\mathrm{E}\left[V_{\mathrm{rel}}(\mathbf{S})\right]=\frac{1}{p-1}\left(p \mathrm{E}\left[\frac{\sum l_{i}^{2}}{\left(\sum l_{i}\right)^{2}}\right]-1\right)
$$

and

$$
\operatorname{Var}\left[V_{\mathrm{rel}}(\mathbf{S})\right]=\left(\frac{p}{p-1}\right)^{2} \operatorname{Var}\left[\frac{\sum l_{i}^{2}}{\left(\sum l_{i}\right)^{2}}\right]
$$

366 In general, moments of the ratio $\sum l_{i}^{2} /\left(\sum l_{i}\right)^{2}$ do not coincide with the ratios of the moments

367 of the numerator and denominator. Specifically under the null hypothesis, however,

and

$$
\begin{gathered}
\mathrm{E}\left[\frac{\sum l_{i}^{2}}{\left(\sum l_{i}\right)^{2}}\right]=\frac{\mathrm{E}\left[\sum l_{i}^{2}\right]}{\mathrm{E}\left[\left(\sum l_{i}\right)^{2}\right]} \\
\mathrm{E}\left[\frac{\left(\sum l_{i}^{2}\right)^{2}}{\left(\sum l_{i}\right)^{4}}\right]=\frac{\mathrm{E}\left[\left(\sum l_{i}^{2}\right)^{2}\right]}{\mathrm{E}\left[\left(\sum l_{i}\right)^{4}\right]}
\end{gathered}
$$

370 hold because of the stochastic independence between $\sum l_{i}^{2} /\left(\sum l_{i}\right)^{2}$ and $\sum l_{i}$ in this special

371 condition (which follows from inspection of the density; John, 1972). Therefore, by use of

372 equations 16,17 , and 20 ,

$$
\begin{aligned}
\mathrm{E}\left[V_{\text {rel }}(\mathbf{S})\right] & =\frac{1}{p-1}\left(p \frac{\mathrm{E}\left[\sum l_{i}^{2}\right]}{\mathrm{E}\left[\left(\sum l_{i}\right)^{2}\right]}-1\right) \\
& =\frac{p+2}{p n+2}
\end{aligned}
$$

374 and

$$
\begin{aligned}
\operatorname{Var}\left[V_{\text {rel }}(\mathbf{S})\right] & =\left(\frac{p}{p-1}\right)^{2}\left(\frac{\mathrm{E}\left[\left(\sum l_{i}^{2}\right)^{2}\right]}{\mathrm{E}\left[\left(\sum l_{i}\right)^{4}\right]}-\left\{\frac{\mathrm{E}\left[\sum l_{i}^{2}\right]}{\mathrm{E}\left[\left(\sum l_{i}\right)^{2}\right]}\right\}^{2}\right) \\
& =\frac{4(p-1)(p+2)(n-1)(n+2)}{(p n+2)^{2}(p n+4)(p n+6)} .
\end{aligned}
$$


376 These results are exact (valid across any $p$ and $n$ ) under multivariate normality.

\section{Correlation matrix}

379 Consider the null hypothesis $\mathbf{P}=\mathbf{I}_{p}$ or $\rho_{i j}=0$ for $i \neq j$. The moments can conveniently be

380 obtained from the form of average squared correlation (eq. 11). It is well known that, under

381 the assumptions of normality and $\rho_{i j}=0$ (for $\left.i \neq j\right), r_{i j}^{2}$ is distributed as

$382 \operatorname{Beta}(1 / 2,(n-1) / 2)$, where $n$ is the degree of freedom (e.g., Anderson, 2003). Therefore,

383 under the null hypothesis,

$$
\operatorname{Var}\left(r_{i j}^{2}\right)=\frac{2(n-1)}{n^{2}(n+2)}
$$

The expectation of $V_{\text {rel }}(\mathbf{R})$ is simply the average:

$$
\mathrm{E}\left[V_{\mathrm{rel}}(\mathbf{R})\right]=\frac{1}{n}
$$

388 This expression is identical to $(p-1)^{-1} \operatorname{Var}\left(l_{i}\right)$ obtainable from Wagner's (1984) results,

389 except for having the degree of freedom $n$ rather than the sample size $N$ in the denominator.

390 This is because Wagner (1984) considered $N$ uncentered observations with mean 0 without explicitly distinguishing $n$ and $N$. Most practical analyses would concern data centered at the sample mean, thus should use $n$ rather than $N$.

394 principle,

$$
\operatorname{Var}\left[V_{\text {rel }}(\mathbf{R})\right]=\frac{4}{p^{2}(p-1)^{2}}\left[\sum_{i<j} \operatorname{Var}\left(r_{i j}^{2}\right)+\sum_{\substack{i<j, k<l,(i, j)<(k, l)}} 2 \operatorname{Cov}\left(r_{i j}^{2}, r_{k l}^{2}\right)\right]
$$


396 where the latter summation is across all non-redundant pairs. However, it is possible to show

$397 \operatorname{Cov}\left(r_{i j}^{2}, r_{k l}^{2}\right)=0$ under the null hypothesis (Appendix C). Therefore, from equations 26 and

39828,

$$
\operatorname{Var}\left[V_{\mathrm{rel}}(\mathbf{R})\right]=\frac{4(n-1)}{p(p-1) n^{2}(n+2)}
$$

400 These expressions are exact for any $p$ and $n$. Schott (2005) proposed a test for independence

401 between sets of normal variables based on these moments.

402

\section{Moments under arbitrary conditions}

\section{Covariance matrix}

405 This section considers moments of eigenvalue dispersion indices under arbitrary

406 covariance/correlation structures and multivariate normality. It is straightforward to obtain

407 the first two moments of $V(\mathbf{S})$ under arbitrary $\boldsymbol{\Sigma}$, provided that moments of relevant terms in

408 equation 12 are available. The results are (Appendix B)

$$
\begin{aligned}
\mathrm{E}[V(\mathbf{S})]= & \frac{n}{p^{2} n_{*}^{2}}\left[(p-n)(\operatorname{tr} \boldsymbol{\Lambda})^{2}+(p n+p-2) \operatorname{tr}\left(\boldsymbol{\Lambda}^{2}\right)\right] \\
= & \frac{n}{p n_{*}^{2}}\left[(p n+p-2) V(\boldsymbol{\Sigma})+(p-1)(p+2)(\operatorname{tr} \boldsymbol{\Lambda})^{2} / p^{2}\right], \\
\operatorname{Var}[V(\mathbf{S})]= & \frac{4 n}{p^{4} n_{*}^{4}}\left\{2(p-n)^{2} \operatorname{tr}\left(\boldsymbol{\Lambda}^{2}\right)(\operatorname{tr} \boldsymbol{\Lambda})^{2}+\left(p^{2} n+p^{2}-4 p+2 n\right)\left[\operatorname{tr}\left(\boldsymbol{\Lambda}^{2}\right)\right]^{2}\right. \\
& +4(p-n)(p n+p-2) \operatorname{tr}\left(\boldsymbol{\Lambda}^{3}\right) \operatorname{tr} \boldsymbol{\Lambda} \\
& \left.+\left(2 p^{2} n^{2}+5 p^{2} n+5 p^{2}-12 p n-12 p+12\right) \operatorname{tr}\left(\boldsymbol{\Lambda}^{4}\right)\right\} .
\end{aligned}
$$

410 The second expression for the expectation comes from the fact $V(\boldsymbol{\Sigma})=\left[p \operatorname{tr}\left(\boldsymbol{\Lambda}^{2}\right)-(\operatorname{tr} \boldsymbol{\Lambda})^{2}\right] /$

$411 p^{2}$, and clarifies that the expectation is a linear function of $V(\Sigma)$. These results are exact, and

412 it is easily verified that they reduce to equations 18 and 21 under the null hypothesis. Profiles

413 of $E[V(\mathbf{S})]$ across a range of $V(\boldsymbol{\Sigma})$ are shown in Figure 2 (top row), under single large 
eigenvalue conditions with varying $p$ and $N$, and fixed $\operatorname{tr} \boldsymbol{\Sigma}$ (detailed conditions are described

under simulation settings below).

417 do not coincide with the ratios of moments under arbitrary $\boldsymbol{\Sigma}$. Here we utilize the following

418 approximations based on the delta method (e.g., Stuart \& Ord, 1994: chapter 10):

and

$$
\mathrm{E}\left(\frac{X}{Y}\right) \approx \frac{\mathrm{E}(X)}{\mathrm{E}(Y)}-\frac{\operatorname{Cov}(X, Y)}{\mathrm{E}(Y)^{2}}+\frac{\mathrm{E}(X) \operatorname{Var}(Y)}{\mathrm{E}(Y)^{3}}
$$

$$
\operatorname{Var}\left(\frac{X}{Y}\right) \approx \frac{\mathrm{E}(X)^{2}}{\mathrm{E}(Y)^{2}}\left[\frac{\operatorname{Var}(X)}{\mathrm{E}(X)^{2}}+\frac{\operatorname{Var}(Y)}{\mathrm{E}(Y)^{2}}-2 \frac{\operatorname{Cov}(X, Y)}{\mathrm{E}(X) \mathrm{E}(Y)}\right]
$$

421 The approximate moments are (Appendix B):

$$
\begin{aligned}
\mathrm{E}\left[\frac{\sum l_{i}^{2}}{\left(\sum l_{i}\right)^{2}}\right] \approx & \frac{(\operatorname{tr} \boldsymbol{\Lambda})^{2}+(n+1) \operatorname{tr}\left(\boldsymbol{\Lambda}^{2}\right)}{n(\operatorname{tr} \boldsymbol{\Lambda})^{2}+2 \operatorname{tr}\left(\boldsymbol{\Lambda}^{2}\right)}-\frac{8(n-1)(n+2)}{n\left[n(\operatorname{tr} \boldsymbol{\Lambda})^{2}+2 \operatorname{tr}\left(\boldsymbol{\Lambda}^{2}\right)\right]^{3}} \\
\times & \left\{n(\operatorname{tr} \boldsymbol{\Lambda})^{3} \operatorname{tr}\left(\boldsymbol{\Lambda}^{3}\right)-n(\operatorname{tr} \boldsymbol{\Lambda})^{2}\left[\operatorname{tr}\left(\boldsymbol{\Lambda}^{2}\right)\right]^{2}-\left[\operatorname{tr}\left(\boldsymbol{\Lambda}^{2}\right)\right]^{3}-2 \operatorname{tr} \boldsymbol{\Lambda} \operatorname{tr}\left(\boldsymbol{\Lambda}^{2}\right) \operatorname{tr}\left(\boldsymbol{\Lambda}^{3}\right)\right. \\
& \left.+3(\operatorname{tr} \boldsymbol{\Lambda})^{2} \operatorname{tr}\left(\boldsymbol{\Lambda}^{4}\right)\right\} ; \\
\operatorname{Var}\left[\frac{\sum l_{i}^{2}}{\left(\sum l_{i}\right)^{2}}\right] \approx & \frac{4(n-1)(n+2)}{n\left[n(\operatorname{tr} \boldsymbol{\Lambda})^{2}+2 \operatorname{tr}\left(\boldsymbol{\Lambda}^{2}\right)\right]^{4}} \\
\times & \left\{n(\operatorname{tr} \boldsymbol{\Lambda})^{4}\left[\operatorname{tr}\left(\boldsymbol{\Lambda}^{2}\right)\right]^{2}+2 n(n+1)(\operatorname{tr} \boldsymbol{\Lambda})^{2}\left[\operatorname{tr}\left(\boldsymbol{\Lambda}^{2}\right)\right]^{3}+2(n+1)\left[\operatorname{tr}\left(\boldsymbol{\Lambda}^{2}\right)\right]^{4}\right. \\
& -4(n-1)(n+2)(\operatorname{tr} \boldsymbol{\Lambda})^{3} \operatorname{tr}\left(\boldsymbol{\Lambda}^{2}\right) \operatorname{tr}\left(\boldsymbol{\Lambda}^{3}\right)+\left(2 n^{2}+3 n-6\right)(\operatorname{tr} \boldsymbol{\Lambda})^{4} \operatorname{tr}\left(\boldsymbol{\Lambda}^{4}\right) \\
& \left.-4 n(\operatorname{tr} \boldsymbol{\Lambda})^{2} \operatorname{tr}\left(\boldsymbol{\Lambda}^{2}\right) \operatorname{tr}\left(\boldsymbol{\Lambda}^{4}\right)-4(\operatorname{tr} \boldsymbol{\Lambda})^{4}\left[\operatorname{tr}\left(\boldsymbol{\Lambda}^{2}\right)\right]^{2}\right\} .
\end{aligned}
$$

423 Inserting these into equation 22 yields the desired moments. The approximate expectation

424 reduces to equation 24 under the null hypothesis, as the higher-order terms cancel out,

425 whereas this is not the case for the approximate variance. Because these expressions are

426 specified by the population eigenvalues alone, they are invariant with respect to orthogonal

427 rotations, as expected from theoretical considerations above. Also, it is easily discerned that

428 these expressions are invariant with respect to uniform scaling of the variables. The accuracy

429 of these approximations will be examined in simulations below. 
430 Profiles of the approximation of $E\left[V_{\text {rel }}(\mathbf{S})\right]$ across a range of $V_{\text {rel }}(\Sigma)$ are shown in

431 Figure 2 (middle row) for the same conditions as above. The profiles are nonlinear; $V_{\text {rel }}(\mathbf{S})$

432 tends to overestimate $V_{\text {rel }}(\boldsymbol{\Sigma})$ when the latter is small, but tends to slightly underestimate

433 when the latter is large. The initial decrease of $E\left[V_{\text {rel }}(\mathbf{S})\right]$ observed in some profiles appears

434 to be an artifact of the approximation.

\section{Correlation matrix}

437 The expectation of $V_{\text {rel }}(\mathbf{R})$ under arbitrary conditions can be obtained from equation 11 with $438 \quad r_{i j}^{2}$ replaced by its expectations, which is known to be (e.g., Soper et al., 1917; Ghosh, 1966;

439 Muirhead, 1982)

$$
\mathrm{E}\left(r_{i j}^{2}\right)=1-\frac{(n-1)\left(1-\rho_{i j}^{2}\right)}{n}{ }_{2} F_{1}\left(1,1 ; \frac{n+2}{2} ; \rho_{i j}^{2}\right), i \neq j,
$$

441 where

$$
{ }_{2} F_{1}(a, b ; c ; z)=\sum_{k=0}^{\infty} \frac{(a)_{k}(b)_{k}}{(c)_{k}} \frac{z^{k}}{k !}
$$

443 is the hypergeometric function, with $(x)_{k}=x(x+1) \ldots(x+k-1)$ denoting rising factorial

444 (formally, $(x)_{k}=\Gamma(x+k) / \Gamma(x)$ with the gamma function $\Gamma(\cdot)$ ). Taking the average of

445 equation 34 across all pairs of variables gives the desired expectation, which is non-

446 asymptotic and exact. It is seen that equation 34 reduces to equation 26 under the null

447 hypothesis.

448 When $p=2$, the exact variance of $V_{\text {rel }}(\mathbf{R})$ is equal to that of the single squared

449 correlation coefficient (Ghosh, 1966):

$$
\operatorname{Var}\left(r_{i j}^{2}\right)=\frac{(n-1)(n+1)\left(1-\rho_{i j}^{2}\right)}{2 n}\left[F-n F^{\prime}-\frac{2(n-1)\left(1-\rho_{i j}^{2}\right)}{n(n+1)} F^{2}\right]
$$

451 where $F={ }_{2} F_{1}\left(1,1 ;(n+2) / 2 ; \rho_{i j}^{2}\right)$ and $F^{\prime}=(F-1) / 2 \rho_{i j}^{2}={ }_{2} F_{1}\left(1,2 ;(n+4) / 2 ; \rho_{i j}^{2}\right) /$

$452(n+2)$; this last form is preferred to avoid numerical instability when $\rho_{i j}^{2}$ is close to 0 . This 
expression reduces to equation 26 under the null hypothesis. When $p>2$, we cannot ignore approach (Appendix D):

$$
\operatorname{Cov}\left(r_{i j}^{2}, r_{k l}^{2}\right) \approx 4 \mathrm{E}\left(r_{i j}\right) \mathrm{E}\left(r_{k l}\right) \operatorname{Cov}\left(r_{i j}, r_{k l}\right)+2\left[\operatorname{Cov}\left(r_{i j}, r_{k l}\right)\right]^{2}
$$

459 Exact and large-sample asymptotic expressions under multivariate normality are available for $460 \mathrm{E}\left(r_{i j}\right)$ and $\operatorname{Cov}\left(r_{i j}, r_{k l}\right)$, respectively (e.g., Soper et al., 1917; Ghosh, 1966; Olkin \& Siotani, 461 1976):

$$
\mathrm{E}\left(r_{i j}\right)=\frac{2}{n}\left[\Gamma\left(\frac{n+1}{2}\right) / \Gamma\left(\frac{n}{2}\right)\right]_{2}^{2} F_{1}\left(\frac{1}{2}, \frac{1}{2} ; \frac{n+2}{2} ; \rho_{i j}^{2}\right)
$$

463 Inserting equations 36-38 to equation 28 yields an approximation for $\operatorname{Var}\left[V_{\text {rel }}(\mathbf{R})\right]$ in

464 arbitrary conditions. In terms of consistency, $\mathrm{E}\left(r_{i j}\right)$ and $\operatorname{Var}\left(r_{i j}^{2}\right)$ could be replaced by

465 respective asymptotic expressions (e.g., Ghosh, 1966; Olkin \& Siotani, 1976), but this does

466 not seem to yield improved accuracy or substantial computational gain.

467 In practice, the variance based on equation 28 can be difficult to calculate for large $p$,

468 because there are $\sim p^{4} / 4$ pairs of correlation coefficients to evaluate. For such cases, the

469 following asymptotic expression based on Konishi's (1979) theory may be more useful

470 (Appendix E):

$$
\operatorname{Var}\left[V_{\mathrm{rel}}(\mathbf{R})\right] \approx \frac{8}{p^{2}(p-1)^{2} n} \sum_{\alpha, \beta=1}^{p} \lambda_{\alpha}^{2} \lambda_{\beta}^{2}\left[\delta_{\alpha \beta}-\left(\lambda_{\alpha}+\lambda_{\beta}\right) \sum_{i=1}^{p} \mathrm{v}_{i \alpha}^{2} \mathrm{v}_{i \beta}^{2}+\sum_{i, j=1}^{p} \rho_{i j}^{2} \mathrm{v}_{i \alpha}^{2} \mathrm{v}_{j \beta}^{2}\right],
$$

472 where $\delta_{i j}$ is the Kronecker delta (equals 1 for $i=j$ and 0 otherwise) and $v_{i \alpha}$ is the $(i, \alpha)$-th

473 element of the population eigenvector matrix $\mathbf{\Upsilon}$. For $p=2$, the accuracy of this expression 
474 can be compared with the exact expression (Fig. 3); visual inspection of the profiles suggest

475 that the accuracy is satisfactory past $N=32-64$, except around $V_{\text {rel }}(\mathbf{P})=0$ where the

476 asymptotic expression diminishes to 0 (as expected from its formula; Appendix E). For $p>2$,

477 accuracies of these approximate expressions are to be evaluated with simulations below.

478 Importantly, the expectation of $V_{\text {rel }}(\mathbf{R})$ is a function of $\rho^{2 \prime}$ s rather than $\Lambda$, and cannot

479 be specified by the latter alone in general. For instance, consider $\left(\begin{array}{ccc}1 & 0.9 & 0 \\ 0.9 & 1 & 0 \\ 0 & 0 & 1\end{array}\right)$ and

$480 \quad\left(\begin{array}{ccc}1 & 0.9 / \sqrt{2} & 0 \\ 0.9 / \sqrt{2} & 1 & 0.9 / \sqrt{2} \\ 0 & 0.9 / \sqrt{2} & 1\end{array}\right)$, both of which are valid correlation matrices. These matrices

481 have identical eigenvalues $\boldsymbol{\Lambda}=\operatorname{diag}(1.9,1.0,0.1)$ and hence an identical value of $V_{\text {rel }}(\mathbf{P})(=$

$4820.27)$, but $\mathrm{E}\left[V_{\text {rel }}(\mathbf{R})\right]$ with $n=10$ are 0.3326 and 0.3156 , respectively. Although the

483 difference diminishes as $n$ increases, this example highlights that the distribution of $V_{\text {rel }}(\mathbf{R})$

484 is partly dependent on population eigenvectors.

485 Profiles of $\mathrm{E}\left[V_{\text {rel }}(\mathbf{R})\right]$ across a range of $V_{\text {rel }}(\mathbf{P})$ are shown in Figure 2 (bottom row),

486 under the same conditions as above. These conditions with single large eigenvalues are

487 special cases in which $\mathrm{E}\left[V_{\text {rel }}(\mathbf{R})\right]$ can be specified by $V_{\text {rel }}(\mathbf{P})$ regardless of eigenvectors

488 (detailed in Appendix A). Indeed, the profiles of the expectations are invariant across $p$ in

489 these special conditions. In some way similar to $V_{\text {rel }}(\mathbf{S}), V_{\text {rel }}(\mathbf{R})$ tends to overestimate and

490 underestimate small and large values of $V_{\text {rel }}(\mathbf{P})$, respectively.

\section{Bias correction}

493 Some authors (Cheverud et al., 1989; Torices \& Muñoz-Pajares, 2015) have suggested

494 correcting the sampling bias in eigenvalue dispersion indices by means of subtracting

495 Wagner's (1984) null expectation from empirical values. This method could potentially be

496 used for $V$ and $V_{\text {rel }}$ with the correct null expectations derived above, to obtain estimators that 
497 is unbiased under the null hypothesis. For $V_{\text {rel }}(\mathbf{S})$ and $V_{\text {rel }}(\mathbf{R})$, however, the subtraction

498 truncates the upper end of the range, potentially compromising interpretability. To avoid this,

499 it might be desirable to scale these indices in a way analogous to the adjusted coefficient of

500 determination in regression analysis (e.g., Cramer, 1987):

501

502

$$
\begin{gathered}
\bar{V}_{\text {rel }}(\mathbf{S})=1-\frac{1-V_{\text {rel }}(\mathbf{S})}{\left.1-E_{\text {null }} V_{\text {rel }}(\mathbf{S})\right]}=\frac{p n+2}{p(n-1)} V_{\text {rel }}(\mathbf{S})-\frac{p+2}{p(n-1)^{\prime}} \\
\bar{V}_{\text {rel }}(\mathbf{R})=1-\frac{1-V_{\text {rel }}(\mathbf{R})}{1-E_{\text {null }}\left[V_{\text {rel }}(\mathbf{R})\right]}=\frac{n}{n-1} V_{\text {rel }}(\mathbf{R})-\frac{1}{n-1},
\end{gathered}
$$

where $\mathrm{E}_{\text {null }}(\cdot)$ denotes expectation under the null conditions (eqs. 24 and 27). This

504 adjustment inflates the variance by the factor of $1 /\left[1-\mathrm{E}_{\text {null }}\left(V_{\text {rel }}\right)\right]^{2}$. Furthermore, these

505 adjusted indices are unbiased only under the null hypothesis (Armbruster et al., 2009; and

506 trivially the case of complete integration), and uniformly underestimate the corresponding

507 population values otherwise (Fig. S2). As the population value gets away from 0 , the adjusted

508 index is outperformed by the unadjusted one in both precision and bias (Fig. S3). It should

509 also be borne in mind that the profiles of expectations are nonlinear and dependent on $N$ (Fig.

510 2). As the adjusted indices will be increasingly conservative for small $N$, it is questionable

511 whether they can be used for comparing samples with different $N$, as originally intended by

512 Cheverud et al. (1989). For these reasons, use of this adjustment would be restricted to

513 estimation of the population value near 0 (up to $0.1-0.2$, depending on $p$ and $N$ ).

514 On the other hand, a global unbiased estimator of $V(\Sigma)$ can be derived from above

515 results:

$$
\begin{aligned}
\tilde{V}(\mathbf{S}) & =\frac{n_{*}^{2}}{n(p n+p-2)}\left(p V(\mathbf{S})-\frac{(p-1)(p+2)}{p^{2}(n-1)(n+2)}\left[(n+1)(\operatorname{tr} \mathbf{S})^{2}-2 \operatorname{tr}\left(\mathbf{S}^{2}\right)\right]\right) \\
& =\frac{1}{p^{2} n(n-1)(n+2)}\left[(p n+2)(\operatorname{tr} \mathbf{A})^{2}-(p+n+1) \operatorname{tr}\left(\mathbf{A}^{2}\right)\right] .
\end{aligned}
$$

517 Its variance can be similarly obtained as 


$$
\begin{aligned}
\operatorname{Var}[\tilde{V}(\mathbf{S})]= & \frac{4}{p^{4} n(n-1)(n+2)}\left\{2(n-1)(n+2) \operatorname{tr}\left(\boldsymbol{\Lambda}^{2}\right)(\operatorname{tr} \boldsymbol{\Lambda})^{2}\right. \\
& +\left(p^{2} n+4 p+2 n+2\right)\left[\operatorname{tr}\left(\boldsymbol{\Lambda}^{2}\right)\right]^{2}-4 p(n-1)(n+2) \operatorname{tr}\left(\boldsymbol{\Lambda}^{3}\right) \operatorname{tr} \boldsymbol{\Lambda} \\
& \left.+\left(2 p^{2} n^{2}+3 p^{2} n-6 p^{2}-4 p n-4\right) \operatorname{tr}\left(\boldsymbol{\Lambda}^{4}\right)\right\},
\end{aligned}
$$

519 which reduces to $4(p-1)(p+2) \sigma^{8} / p^{3} n(n-1)(n+2)$ under the null hypothesis.

520 Comparison with equations 21 and 30 suggests that this variance is smaller than that of $V(\mathbf{S})$,

521 especially under the null hypothesis. Therefore, $\tilde{V}(\mathbf{S})$ seems superior in both precision and

522 bias and can be used when estimation of $V(\boldsymbol{\Sigma})$ is desired. It can be used to compare multiple

523 samples, provided that its sensitivity to overall scaling is not of concern, e.g., comparison

524 between closely related taxa.

\section{Simulation}

\section{Methods}

528 Simulations were conducted under various conditions in order to understand sampling

529 properties of the eigenvalue dispersion indices. All simulations were done assuming

530 multivariate normality, with varying population covariance matrix $\boldsymbol{\Sigma}$, number of variables $p$

$531(=2,4,8,16,32,64,128,256$, and 1024), and sample size $N(=4,8,16,32,64,128$, and

532 256).

533 For every $p$, the following population eigenvalue conformations were considered: 1)

534 the null condition, 2) $q$-large $\lambda$ conditions, 3) a linearly decreasing $\lambda$ condition, and 4) a

535 quadratically decreasing $\lambda$ condition (see Fig. 4 for examples). The null condition is where all

536 population eigenvalues are equal in magnitude $\left(V_{\text {rel }}(\Sigma)=0\right)$, corresponding to the null

537 hypothesis of sphericity (Fig. 4A). The $q$-large $\lambda$ conditions are where the first $q(=1,2$, and

5384 , provided $p>q$ ) population eigenvalues are equally large and the remaining $p-q$ ones are 
equally small $\left(\lambda_{1}=\cdots=\lambda_{q}>\lambda_{q+1}=\cdots=\lambda_{p}\right)$, with varying $V_{\text {rel }}(\Sigma)(=0.1,0.2,0.4,0.6$,

540 and 0.8 ; Fig. 4B-G). The necessary condition $\lambda_{p} \geq 0$ constrains possible combinations of $q$

541 and $V_{\text {rel }}(\boldsymbol{\Sigma})$ : the possible choices of $V_{\text {rel }}(\boldsymbol{\Sigma})$ are $0.1-0.8,0.1-0.4$, and $0.1-0.2$ for $q=1,2$, and

5424 , respectively (Appendix A). These conditions are intended to represent hypothetical

543 situations where only a few components of meaningful signals are present in the population.

544 Individual eigenvalues were calculated for each combination of $p, q$, and $V_{\text {rel }}(\boldsymbol{\Sigma})$ as described

545 in Appendix A. The linearly and quadratically decreasing $\lambda$ conditions are where the

546 population eigenvalues are linearly and quadratically, respectively, decreasing in magnitude

547 (Fig. 4H; Appendix A), in which cases the value of $V_{\text {rel }}(\Sigma)$ is fixed for a given $p$. These

548 conditions are intended to represent covariance structures with gradually decreasing signals.

549 One might claim that some of these situations, especially $q$-large $\lambda$ conditions, are too

550 simplistic and biologically unrealistic, but these simple settings enable us to clarify

551 systematic relationships between parameters and sampling properties. The primary aim here

552 is to explore sampling properties across a wide range of parameters, rather than focusing on

553 biologically "realistic" regions (which would depend on specific organismal systems). It

554 should also be recalled that sampling error alone can yield gradually decreasing patterns of

555 sample eigenvalues typically observed in empirical datasets (see above and below).

556 For sake of simplicity, all population covariance matrices were scaled to ensure

$557 V(\boldsymbol{\Sigma})=V_{\text {rel }}(\boldsymbol{\Sigma})$; that is, $\operatorname{tr} \boldsymbol{\Sigma}=p(p-1)^{-1 / 2}$. This scaling also makes the magnitude of $V(\boldsymbol{\Sigma})$

558 comparable across varying $p$. In addition, a population covariance matrix $\boldsymbol{\Sigma}$ was constructed

559 from a predefined set of eigenvalues such that its diagonal elements are equal: $\sigma_{i i}=\bar{\lambda}=$

$560(p-1)^{-1 / 2}$ for all $i$, thereby enforcing $\Sigma=(p-1)^{-1 / 2} \mathbf{P}$. This construction allows for

561 examining both covariance and correlation matrices with the same population $V_{\text {rel }}$ from a

562 single simulated dataset, saving computational resources. $\boldsymbol{\Sigma}$ was constructed from $\boldsymbol{\Lambda}$ by the

563 iterative Givens rotation algorithm of Davies \& Higham (2000), which is guaranteed to 
564 converge within $p-1$ iterations. This algorithm was implemented as coded by Waller (2020),

565 but with the following modifications for reproducibility: no random orthogonal rotation was

566 involved at the initial stage, and rotation axes were chosen in a fixed order. It should be noted

567 that the rotations involved—choice of eigenvectors-would in general influence distributions

568 of $V_{\text {rel }}(\mathbf{R})$, except for certain special cases including the 1-large $\lambda$ condition (Appendix A). It

569 is impractical to exhaustively examine numerous possible conformations of eigenvectors, so

570 only the single conformation generated by this algorithm was used for each combination of

571 parameters.

572 The eigenvalues of a sample covariance matrix were obtained from singular value

573 decomposition of the data matrix, as the singular values squared and then divided by $n_{*}$ (see,

574 e.g., Jolliffe, 2002). When $p>N-1,0$ 's were appended to this vector so that $p$ sample

575 eigenvalues were present. Data were centered at the sample mean before the decomposition,

576 therefore $n=N-1$. It was chosen that $n_{*}=n$. The eigenvalues for a sample correlation

577 matrix were obtained similarly from the sample-mean-centered data scaled with the sample

578 standard deviation for each variable.

579 To summarize, each set of simulations consists of the following steps: 1) define a

580 desired set of eigenvalues $\Lambda ; 2$ ) construct the population covariance matrix $\boldsymbol{\Sigma}$ with the

581 rotation algorithm explained above; 3) generate $N$ i.i.d. normal observations from $N_{p}(\mathbf{0}, \boldsymbol{\Sigma})$;

582 4) eigenvalues of sample covariance and correlation matrices were obtained from singular

583 value decomposition of the sample-mean-centered data; 5) $V(\mathbf{S}), V_{\text {rel }}(\mathbf{S})$, and $V_{\text {rel }}(\mathbf{R})$ were

584 calculated from the eigenvalues; 6) the steps 3-5 were iterated for 5,000 times in total with

585 the same $N$ and $\boldsymbol{\Sigma}$. The simulations were conducted on the $\mathrm{R}$ environment version 3.5.3 (R

586 Core Team, 2019). The function "genhypergeo" of the package "hypergeo" (Hankin, 2015)

587 was used to evaluate the hypergeometric function in the moments of $V_{\text {rel }}(\mathbf{R})$. The time-

588 consuming calculation of $\operatorname{Var}\left[V_{\text {rel }}(\mathbf{R})\right]$ from equations 28 and 36-38 was aided by a $\mathrm{C}++$ 
589 code via the package "Rcpp" (Eddelbuettel \& Balamuta, 2018). The codes are provided as

590 Supplementary Material.

591

\section{Results}

593 Examined individually, sample eigenvalues were biased estimators of population eigenvalues,

594 as expected. Selected eigenvalue distributions of sample covariance and correlation matrices

595 are shown in Figures 4 and S1, respectively. Typically, the first few eigenvalues were

596 overestimated, with the rest being underestimated. Note that gradually decreasing scree-like

597 profiles of sample eigenvalues typical of empirical datasets can arise even when most

598 population eigenvalues are identical in magnitude. The sampling biases decreases as $N$

599 increases. These overall trends were similarly observed for correlation matrices, although the

600 upper tail of the largest eigenvalue tended to be truncated for correlation matrices because of

601 the constraint tr $\mathbf{R}=p$, effectively cancelling the tendency of overestimation in this

602 eigenvalue (Fig. S1).

603 Sampling distributions of $V(\mathbf{S})$ are shown in Figures 5 and S4-S6, and their summary

604 statistics are shown in Tables 1 and S1. Distributions were unimodal but highly skewed with

605 long upper tails, especially when $N$ or $p$ is small. As expected, sampling dispersion decreases

606 consistently with increasing $N$, with skewness decreasing at the same time. Interestingly, the

607 shape of distribution does not visibly change with increasing $p$, at least with moderately large

$608 N(\geq 32$, say). In all conditions, $V(\mathbf{S})$ tended to overestimate the population value $V(\boldsymbol{\Sigma})$.

609 Increasing $V(\boldsymbol{\Sigma})$ drastically increased sampling dispersion and skewness, whereas increasing

$610 q$ with a fixed $V(\boldsymbol{\Sigma})$ decreased sampling dispersion without changing the mean as much.

611 Sampling distributions of $V(\mathbf{S})$ under linearly and quadratically decreasing $\lambda$ conditions look

612 similar to those under $q$-large $\lambda$ conditions with similar $V(\Sigma)$ values for the respective $p$. The

613 expressions of the expectation and variance of $V(\mathbf{S})$ almost always coincided with the 
614 sampling mean and variance within a reasonable range of random fluctuations (as expected,

615 since those results are exact).

616 Results for $V_{\text {rel }}(\mathbf{S})$ are summarized in Figures 6 and S6-S8 and Tables 2 and S2.

617 Distributions were unimodal within the range $(0,1)$, except when $N=4$ and $p=2$ where the

618 distribution was essentially uniform. As was the case for $V(\mathbf{S})$, the sampling dispersion of

$619 V_{\text {rel }}(\mathbf{S})$ decreased drastically with increasing $N$, and to some extent with increasing $p$, while

620 the shape of distribution does not seem to change remarkably with increasing $p$ past certain $N$.

$621 V_{\text {rel }}(\mathbf{S})$ tended to overestimate the population value $V_{\text {rel }}(\Sigma)$, except when the latter is rather

622 large $(=0.8)$ where slight underestimation was observed. With increasing $q$ for a fixed

$623 V_{\text {rel }}(\Sigma)$, the distributions tended to shrink, but the sampling bias remained virtually

624 unchanged or slightly increased. In the null conditions, the exact expressions of the

625 expectation and variance performed perfectly (as expected). The approximate expectation for

626 arbitrary conditions derived above yielded substantially smaller values than the empirical

627 means when $N$ is small; however, the approximation worked satisfactorily with moderate $N$

628 ( $\geq 16-32)$, with the deviations from empirical means mostly falling within 2 standard error

629 units. In addition, the approximate expectation worked rather well, even with small $N$, under

630 either A) the $q$-large $\lambda$ conditions with $q=2$ and $V_{\text {rel }}(\Sigma)=0.4$, B) same with $q=4$, or C)

631 linearly and quadratically decreasing $\lambda$ conditions with moderately large $p(\geq 16)$. Other

632 conditions held constant, the accuracy of the approximate expectation in absolute scale

633 tended to slightly improve with increasing $p$, effectively balancing with the decreasing

634 sampling dispersion, so that the relative bias in standard error unit remains almost invariant

635 across varying $p$. The approximate variance for arbitrary conditions derived above yielded

636 substantially larger values than the empirical variance, except under the $q$-large $\lambda$ conditions

637 with $q=1$ and $V_{\text {rel }}(\Sigma)=0.8$ where it yielded smaller values. Nevertheless, with moderately

638 large $N(\geq 64)$, the inaccuracy typically decreased to $<5 \%$ in the scale of standard deviation 
639 (SD scale hereafter), except under the linearly and quadratically decreasing $\lambda$ conditions with

640 moderately large $p(\geq 16)$, where it was more accurate.

641 Results for $V_{\text {rel }}(\mathbf{R})$ are summarized in Figures 7, S6, S9, and S10 and Tables 3 and S3.

642 Distributions were unimodal within the range $(0,1)$, except when $p=2$ and $N \leq 8$ where an

643 additional peak is usually present near 0 . The overall response to varying $p$ and $N$ is largely

644 similar to that of $V_{\text {rel }}(\mathbf{S})$, although the shape of distribution was substantially different for

645 small $N$. As expected from the theoretical expectations noted above, $V_{\text {rel }}(\mathbf{R})$ tends to

646 overestimate the population value $V_{\text {rel }}(\mathbf{P})$ when the latter is small but tends to underestimate

647 it when $V_{\text {rel }}(\mathbf{P})=0.8$. The expressions of expectation for the null and arbitrary conditions

648 and variance for the null condition derived above showed almost perfect match with the

649 empirical means and variances (as expected). The heuristic approximation of the variance of

$650 V_{\text {rel }}(\mathbf{R})$ for arbitrary conditions using equations 28 and 36-38 yielded larger values than the

651 empirical variances in all cases, except when $V_{\text {rel }}(\mathbf{P})=0.8$ where slight underestimation was

652 observed. In all cases, the error of this expression decreased to $\sim 0-2 \%$ in the SD scale-

653 statistically indistinguishable from random fluctuation with 5000 iterations-for moderately

654 large $N$ (typically $\geq 64-128$, occasionally $\geq 16-32$ ). The asymptotic variance of $V_{\text {rel }}(\mathbf{R})$ with

655 equation 39 behaved more idiosyncratically. It yielded similar values to the previous

656 expression, overestimating the true values, under A) the $q$-large $\lambda$ conditions with $q=1$ and

$\left.657 V_{\text {rel }}(\mathbf{P})=0.1-0.6, \mathrm{~B}\right)$ same with $q=2$ and $V_{\text {rel }}(\mathbf{P})=0.1-0.2$ except when $p=4$, and C)

658 the quadratically decreasing $\lambda$ conditions with $p=4$; whereas it yielded smaller values than

659 the true values under a) the $q$-large $\lambda$ conditions with $q=1$ and $V_{\text {rel }}(\mathbf{P})=0.8$, b) same with

$660 q=2$ and $\left.V_{\text {rel }}(\mathbf{P})=0.4, \mathrm{c}\right)$ same with $q=4$, d) same with $q=2$ and $\left.p=4, \mathrm{e}\right)$ the linearly

661 decreasing $\lambda$ conditions, and f) the quadratically decreasing $\lambda$ conditions except when $p=4$.

662 This expression was more accurate than the previous one in the cases a and b, but more

663 inaccurate in other cases. Under some conditions, relative error can be extremely large (10- 
$664300 \%$ in SD scale with $N=256$ ), especially when the smallest population eigenvalue was

665 small in magnitude $(<0.1)$. A practical advantage of this approximation over the previous one

666 may lie in the computational resource required for large $p$. With the present $\mathrm{R}$

667 implementation, evaluation of this expression is faster by a factor of thousands than that of

668 the previous one for a correlation matrix with $p=1024(\sim 1$ versus $\sim 1500$ CPU seconds on a

669 regular desktop PC), as the amount of computation increases drastically as $p$ grows (the latter

670 took only $\sim 5$ CPU seconds for $p=256$ ).

\section{Discussion}

673 Eigenvalue dispersion indices can be calculated for covariance or correlation matrices in

674 similar ways, but implications are rather different. On the one hand, the relative eigenvalue

675 variance of a sample covariance matrix $V_{\text {rel }}(\mathbf{S})$ is a test statistic for sphericity (John, 1972;

676 Sugiura, 1972; Nagao, 1973), and is thus interpreted as a measure of eccentricity of variation,

677 be it due to large variation of a single trait or covariation between traits. Interpretation of the

678 unstandardized eigenvalue variance of a sample covariance matrix $V(\mathbf{S})$ is less

679 straightforward, but it can potentially be useful in comparing eccentricity between samples

680 when the sensitivity to overall scaling is not of concern, primarily for the presence of an

681 unbiased estimator of the corresponding population value with a known variance (eq. 41). On

682 the other hand, the relative eigenvalue variance of a sample correlation matrix $V_{\text {rel }}(\mathbf{R})$ is

683 identical to the average of the squared correlation coefficients across all pairs of traits

684 (Durand \& Le Roux, 2017; see above). The average squared correlation is another commonly

685 used index of phenotypic integration (e.g., Cheverud et al., 1983), but its equivalence to

$686 V_{\text {rel }}(\mathbf{R})$ seems to have been overlooked, apart from an empirical confirmation by Haber's

687 (2011) simulations. Obviously, the choice between covariance and correlation should be 688 made according to the scope of individual analyses (Klingenberg, 1996; Hansen \& Houle, 
689 2008; Pavlicev et al., 2009; Goswami \& Polly, 2010; see also Machado et al., 2019 for an

690 interesting discussion). Usual caveats for the choice between covariance and correlation is

691 also pertinent here (Jolliffe, 2002): covariance between traits have clear interpretability only

692 if all traits are in the same unit. This is despite that $V_{\text {rel }}(\mathbf{S})$ is dimensionless and independent

693 of the overall scaling of traits.

694 Perhaps the most remarkable finding of this study is that the distributions of $V_{\text {rel }}(\mathbf{S})$

695 and $V_{\text {rel }}(\mathbf{R})$ do not seem to vary much with the number of variables $p$ itself. The above

696 expressions for the (approximate) mean and variance can be calculated for any $p$, and

697 simulation results indicate that their accuracy are not compromised by large $p$ (Figs. 5-7 and

698 S4-S10; Tables 1-3 and S1-S3). This finding highlights potential applicability of these

699 measures to high-dimensional phenotypic data. Nevertheless, it should be remembered that,

700 when $p$ exceeds the degree of freedom $n, p-n$ of the sample eigenvalues are 0 and hence

701 the corresponding population eigenvalues are not estimable. In addition, the first sample

702 eigenvector tends to be consistently diverged from the first population eigenvector in high-

703 dimensional settings (Johnstone, 2007; Johnstone \& Paul, 2018).

\section{Applications and limitations}

706 The present analytic results assume simple independent sampling from a multivariate normal

707 population and the Wishart-ness of the cross-product matrix. For some biological datasets,

708 certain modifications would be required. A simple example is data consisting of multiple

709 groups with potentially heterogeneous means, e.g., intraspecific variation calculated from

710 multiple geographic populations or sexes. If uniform $\boldsymbol{\Sigma}$ across groups can be assumed, cross-

711 product matrices from the data centered at the respective group's sample mean can be

712 summed across groups to obtain a pooled cross-product matrix, which is, by the additivity of

713 Wishart variables, distributed as $W_{p}(\Sigma, N-g)$, where $N$ is the total sample size and $g$ is the 
714 number of groups. That is, all above expressions can be applied by simply using the degree of

715 freedom $N-g$. A similar correction is required when eigenvalue dispersion indices are

716 applied to partial correlation matrices (Torices \& Méndez, 2014; Torices \& Muñoz-Pajares,

717 2015). The distribution of sample partial correlation coefficients in $p_{1}$ variables

718 conditionalized on $p_{2}$ other variables based on $N$ observations is the same as that of ordinary

719 correlation coefficients based on $N-p_{2}$ observations with the same corresponding

720 parameters (e.g., Anderson, 2003: p. 143), so the appropriate degree of freedom is $n-p_{2}$.

721 Both of these procedures are essentially to examine the covariance/correlation matrix of

722 residuals after conditionalizing on covariates.

723 Present analytical results may not be applicable to those empirical covariance or

724 correlation matrices that are not based on a Wishart matrix. Primary examples are the

725 empirical $\mathbf{G}$ matrices estimated from variance components in MANOVA designs or obtained

726 as likelihood-based estimators in mixed models (e.g., Lynch \& Walsh, 1998; Meyer \&

727 Kirkpatrick, 2005). Mean-standardization, a method recommended for analyzing $\mathbf{G}$ matrices

728 (Houle, 1992; Hereford et al., 2004; Hansen \& Houle, 2008), can also violate the

729 distributional assumption if sample means are used in the standardization. If eigenvalue

730 dispersion indices are to be used with any of these methods, their sampling properties need to

731 be critically assessed (see also Sztepanacz \& Blows, 2017).

732 The assumption of multivariate normality may be intrinsically inappropriate for some

733 types of data, including meristic (count) data, compositional or proportional data, angles, and

734 directional data. Application of eigenvalue dispersion indices (or indeed

735 covariance/correlation itself) to such data types would require special treatments, which are

736 beyond the scope of this study. Needless to say, the appropriateness of multivariate normality

737 should be critically assessed in every empirical dataset when the present analytic results are 
738 to be applied, even if the data type is conformable with normality. Robustness of the above

739 results against non-normality may deserve some investigations.

740

\section{Shape variables}

742 The application to traditional morphometric datasets, in which all variables are typically

743 measured in the same unit, is rather straightforward, as covariance/correlation in such

744 variables has full interpretability in the Euclidean trait space. Quite often, component(s) of

745 little interest, e.g., size, are removed by transforming raw data, inducing covariation in

746 resultant variables that needs to be taken into account in hypothesis tests. The most typical

747 transformation is the division by an isometric or allometric size variable (Jolicoeur, 1963;

748 Mosimann, 1970; Mosimann \& James, 1979; Darroch \& Mosimann, 1985; Klingenberg,

749 1996, 2016), which can conveniently be done by orthogonal projection in the space of log-

750 transformed variables. The projection of objects onto the hyperplane orthogonal to a subspace,

751 say, the column space of $\mathbf{H}(p \times k$ full-column-rank matrix; for the isometric size vector,

$752 \mathbf{H}=p^{-1 / 2} \mathbf{1}_{p}$ ), can be done by right-multiplying the data by the projection matrix (e.g.,

753 Burnaby, 1966):

$$
\mathbf{I}_{p}-\mathbf{H}\left(\mathbf{H}^{T} \mathbf{H}\right)^{-1} \mathbf{H}^{T}
$$

754 Therefore, the covariance matrix in the resultant space can be obtained from that in the

755 original space $\Sigma$ as

$$
\left[\mathbf{I}_{p}-\mathbf{H}\left(\mathbf{H}^{T} \mathbf{H}\right)^{-1} \mathbf{H}^{T}\right] \Sigma\left[\mathbf{I}_{p}-\mathbf{H}\left(\mathbf{H}^{T} \mathbf{H}\right)^{-1} \mathbf{H}^{T}\right]
$$

756 Under the null condition $\left(\boldsymbol{\Sigma}=\sigma^{2} \mathbf{I}_{p}\right)$ specifically, this becomes

$$
\sigma^{2}\left[\mathbf{I}_{p}-\mathbf{H}\left(\mathbf{H}^{T} \mathbf{H}\right)^{-1} \mathbf{H}^{T}\right]
$$

757 because the projection matrix is symmetric and idempotent. This transformation renders $k$

758 eigenvalues to be 0 by construction. When the focus is on covariance rather than correlation,

759 these null eigenvalues can optionally be dropped from calculation of eigenvalue mean and 
760 dispersion, so that the resultant dispersion index quantifies eccentricity of variation in the

761 subspace of interest. Theories derived above can be applied with minimal modifications,

762 although the asymptotic variance of $V_{\text {rel }}(\mathbf{R})$ (eq. 39) may not work well due to singularity.

763 These discussions assume that independence between the raw variables can at least

764 hypothetically be conceived, e.g., when measurements are taken from non-overlapping parts

765 of an organism. If measurements are taken from overlapping parts of an organism, then the

766 dependence between variables due to the geometric configuration needs to be taken into

767 consideration on a case-by-case basis (Mitteroecker et al., 2012).

768 Application to landmark-based geometric morphometric data is more complicated,

769 primarily because the shape space of Procrustes-aligned landmark configurations is (typically

770 a restricted region of) the surface of a hyper(hemi)sphere (e.g., Slice, 2001). In practice,

771 however, empirical analyses are usually conducted on a Euclidean tangent space instead of

772 the shape space itself, assuming that the former gives a satisfactory metric approximation of

773 the latter (e.g., Rohlf, 1999; Marcus et al., 2000; Klingenberg, 2020). It will in principle be

774 possible to obtain an approximate population covariance matrix of landmark coordinates in

775 this tangent space from a hypothetical covariance matrix of raw landmark coordinates before

776 alignment, by using the orthogonal projection method mentioned above with such an $\mathbf{H}$

777 whose columns represent the non-shape components. Such a set of vectors can be obtained

778 either as a basis of the complement of the tangent space (see Rohlf \& Bookstein, 2003) or

779 directly from the consensus configuration (Klingenberg, 2020). The stereographic projection

780 might potentially be preferred over the orthogonal projection in projecting aligned empirical

781 configurations in the shape space to the tangent space-not to be confused with the projection

782 from the raw space to the tangent space-for purposes of analysing eccentricity of variation.

783 This is because the stereographic projection tends to approximately preserve multivariate

784 normality of the raw coordinates into the resultant tangent space, provided that the variation 
785 in the raw coordinates is sufficiently small and that the mean configuration is taken as the

786 point of tangency (Rohlf, 1999). It should be noted that Procrustes superimposition changes

787 perceived patterns of variation in landmark coordinates, often rather drastically (Rohlf \&

788 Slice, 1990; Walker, 2000). Such phenomena are probably to be seen as properties of shape

789 variables, rather than necessarily nuisance artifacts (Klingenberg, 2021). Whether these can

790 be of concern or not would depend on the scope of individual analyses (see also Machado et

791 al., 2019).

792

\section{Phylogenetic data}

794 So far data were assumed to be i.i.d. multivariate normal variables. Important applications in

795 evolutionary biology involve non-i.i.d. observations, most notably phylogenetically

796 structured data in which $N$ observations (typically species) have covariance due to shared

797 evolutionary histories. Trait covariation at the interspecific level may have interpretations

798 under certain evolutionary models (Felsenstein, 1988; Hansen \& Martins, 1996; Revell \&

799 Harmon, 2008; Uyeda \& Harmon, 2014; Caetano \& Harmon, 2019). Under the assumption

800 that trait evolution along phylogeny can be described by (potentially a mixture of) linear

801 invariant Gaussian models, such as the Brownian motion (BM), accelerating-decelerating

802 (ACDC; or early burst), and Ornstein-Uhlenbeck (OU) processes, the joint distribution of the

803 observations is known to be multivariate normal (Hansen \& Martins, 1996; Manceau et al.,

804 2017; Mitov et al., 2020). A brief overview is given below for potential applications of the

805 present analytic results to phylogenetically structured data.

806 For BM and its modifications, including BM with a trend, Pagel's $\lambda$, and ACDC

807 models, the covariance matrix of the $N \times p$ dimensional data $\mathbf{X}$ can be factorized into the

808 intertrait and interspecific components in the form of Kronecker product: $\boldsymbol{\Sigma} \otimes \boldsymbol{\Psi}$, where $\boldsymbol{\Psi}$ is

809 the $N \times N$ interspecific covariance matrix specified by the underlying phylogeny and 
810 parameter(s) specific to the evolutionary model (see Hansen \& Martins, 1996; Freckleton et

811 al., 2002; Blomberg et al., 2003; Clavel et al., 2015; Mitov et al., 2020). In this case the data

812 can conveniently be considered as a matrix-variate normal variable (see Gupta \& Nagar,

813 1999): $\mathbf{X} \sim N_{N, p}(\mathbf{M}, \boldsymbol{\Sigma} \otimes \mathbf{\Psi})$, where $\mathbf{M}$ is a $N \times p$ matrix of means. If $\boldsymbol{\Psi}$ is known a priori-

814 that is, we have an accurate phylogenetic hypothesis and parameters - the change of variables

$815 \mathbf{Y}=\Psi^{-1 / 2} \mathbf{X}$ leads to $\mathbf{Y} \sim N_{N, p}\left(\Psi^{-1 / 2} \mathbf{M}, \boldsymbol{\Sigma} \otimes \mathbf{I}_{N}\right)$, thereby essentially avoiding the

816 complication of dependence between observations. This procedure is widely recognized as

817 the (phylogenetic) generalized least squares (GLS; e.g., Grafen, 1989; Martins \& Hansen,

818 1997; Rohlf, 2001; Symonds \& Blomberg, 2014). If we know the population mean M in

819 addition, then the cross-product matrix centered at it,

$$
\left(\mathbf{Y}-\Psi^{-1 / 2} \mathbf{M}\right)^{T}\left(\mathbf{Y}-\Psi^{-1 / 2} \mathbf{M}\right)=(\mathbf{X}-\mathbf{M})^{T} \Psi^{-1}(\mathbf{X}-\mathbf{M})
$$

820 is distributed as $W_{p}(\boldsymbol{\Sigma}, N)$. If we don't exactly know $\mathbf{M}$ yet still assume $\mathbf{M}=\mathbf{1}_{N} \boldsymbol{\mu}^{T}$ with the

821 unknown but uniform $p \times 1$ mean vector $\boldsymbol{\mu}$, then the GLS estimate of the mean $\widehat{\boldsymbol{\mu}}=$

$822\left(\mathbf{1}_{N}^{T} \boldsymbol{\Psi}^{-1} \mathbf{1}_{N}\right)^{-1} \mathbf{1}_{N}^{T} \boldsymbol{\Psi}^{-1} \mathbf{X}$ (e.g., Martins \& Hansen, 1997) can be used to obtain a sample-

823 mean-centered cross-product matrix

$$
\left(\mathbf{Y}-\boldsymbol{\Psi}^{-1 / 2} \mathbf{1}_{N} \widehat{\boldsymbol{\mu}}^{T}\right)^{T}\left(\mathbf{Y}-\Psi^{-1 / 2} \mathbf{1}_{N} \widehat{\boldsymbol{\mu}}^{T}\right)=\left(\mathbf{X}-\mathbf{1}_{N} \widehat{\boldsymbol{\mu}}^{T}\right)^{T} \boldsymbol{\Psi}^{-1}\left(\mathbf{X}-\mathbf{1}_{N} \widehat{\boldsymbol{\mu}}^{T}\right),
$$

824 which can be shown to be distributed as $W_{p}(\Sigma, N-1)$. If there are multiple blocks of species

825 with different means (regimes), then cross-product matrices calculated separately for each of

826 these can be summed to obtain a Wishart matrix with a modified degree of freedom as

827 mentioned above, although it would need to be asked first whether those regimes share the

828 same $\boldsymbol{\Sigma}$ (Revell \& Collar, 2009; Caetano \& Harmon, 2019). The present analytic results can

829 directly be applied to these Wishart matrices. Estimation of $\boldsymbol{\Sigma}$ based on this transformation

830 has previously been devised (Revell \& Harmon, 2008; see also Huelsenbeck \& Rannala,

831 2003, Revell \& Harrison, 2008; Adams \& Felice, 2014), and has been shown to have superior 
832 accuracy in estimating eigenvalues over estimation ignoring phylogenetic structures under

833 model conditions (Revell, 2009). Variants of this method have already been applied to

834 analyze eccentricity of interspecific covariation (Haber, 2016; Watanabe, 2018). In practice,

835 however, $\boldsymbol{\Psi}$ is virtually never known exactly because phylogeny and parameters of

836 evolutionary models are generally estimated with error, so empirical cross-product matrices

837 may not be strictly Wishart. This source of error is inherent to any phylogenetic comparative

838 analysis. Unlike the GLS estimate of the mean, which remains unbiased even when $\boldsymbol{\Psi}$ is

839 misspecified, the GLS estimate of trait (co)variance is in general biased in this case (see

840 Rohlf, 2006). Although there are certain ways to incorporate phylogenetic uncertainty into

841 statistical inferences (e.g., Huelsenbeck \& Rannala, 2003; Garamszegi \& Mundry, 2014;

842 Nakagawa \& de Villemereuil, 2019), potential consequences of the uncertainty over the

843 distributions of derived statistics require further investigation (see also Revell et al., 2018).

844 Nevertheless, the GLS estimation with slightly inaccurate $\boldsymbol{\Psi}$ is supposed to yield a better

845 estimate of trait (co)variance than the estimation ignoring phylogenetic covariation altogether

846 (Rohlf, 2006). It should be noted that uniform scaling of $\boldsymbol{\Psi}$ translates to the reciprocal scaling

847 of the cross-product matrix; $V(\mathbf{S})$ is sensitive to this scaling, whereas $V_{\text {rel }}(\mathbf{S})$ and $V_{\text {rel }}(\mathbf{R})$ are

848 not. Therefore, specifically under the BM model, the phylogenetic uncertainty would be the

849 only major concern for the latter two indices.

850 Unfortunately, the GLS estimation of trait covariance does not seem feasible for

851 multivariate OU models, where the joint covariance matrix cannot in general be factorized

852 into intertrait and interspecific components (Bartoszek et al., 2012; Mitov et al., 2020). This

853 is notably except when the selection strength matrix is spherical and the tree is ultrametric, in

854 which case a factorization of the form $\boldsymbol{\Sigma} \otimes \boldsymbol{\Psi}$ is possible (the scalar OU model; Bastide et al.,

855 2018) and hence the GLS cross-product matrix can in principle be calculated, assuming that

856 the relevant parameters are known. Otherwise, the random drift/diffusion matrix of the OU 
model estimated in one or other criteria can potentially be analyzed, although little is known

858 about its sampling properties under various implementations, other than that accurate

859 estimation is notoriously difficult (e.g., Ho \& Ané, 2014; Clavel et al., 2015). Further studies

860 are required on technical aspects of quantifying trait covariation in phylogenetically

861 structured data under such complex models, as well as its biological implications (e.g.,

862 Adams \& Collyer, 2018, 2019b; Mitov et al., 2019, 2020; Clavel et al., 2019; Clavel \&

863 Morlon, 2020).

864

\section{Concluding remarks}

866 Eigenvalue dispersion indices of covariance or correlation matrices are commonly used as

867 measures of trait covariation, but their statistical implications have not been well appreciated

868 by biologists, against which criticism has reasonably been directed (Hansen \& Houle, 2008;

869 Blows \& McGuigan, 2015; Hansen et al., 2019). As discussed above, $V_{\text {rel }}(\mathbf{S})$ and $V_{\text {rel }}(\mathbf{R})$

870 have clear statistical justifications as test statistics for sphericity and no correlation,

871 respectively. However, sample eigenvalue dispersion indices are biased estimators of the

872 corresponding population values. This paper derived (or restated) exact and approximate

873 expressions for the expectation and variance of $V(\mathbf{S}), V_{\text {rel }}(\mathbf{S})$, and $V_{\text {rel }}(\mathbf{R})$ under the

874 respective null and arbitrary conditions, with which empirical values can be compared. All

875 null moments are exact, as well as both moments of $V(\mathbf{S})$ and the expectation of $V_{\text {rel }}(\mathbf{R})$

876 under arbitrary conditions. Moments of $V_{\text {rel }}(\mathbf{S})$ under arbitrary conditions are approximations

877 based on the delta method; the approximate expectation was shown to work reasonably well

878 with a moderate sample size $(N \geq 16-32)$, whereas the approximate variance requires a

879 larger sample size to be reliable (e.g., $N \geq 64$, depending on other conditions). Two

880 approximate expressions were given for the variance of $V_{\text {rel }}(\mathbf{R})$ under arbitrary conditions.

881 The one with equations 28 and 36-38 works reasonably well with a moderate to large sample 
882 size $(N \geq 16-128)$ but requires nontrivial computational time for a large matrix (e.g.,

$883 p=1024$ ), whereas the one with equation 39 tends to be more inaccurate in some conditions

884 but can be evaluated almost instantly. Under such conditions where these expressions work,

885 they can be used for (approximate) statistical inferences and hypothesis tests for the

886 magnitude of integration, as well as for determination of appropriate sample sizes in

887 empirical analyses, essentially replacing qualitative thresholds proposed earlier (e.g., Haber,

888 2011; Jung et al., 2020).

889 There are several conceivable ways for statistical inferences and hypothesis testing for

890 eigenvalue dispersion indices. When sample size is so large that distributions of the indices

891 are virtually symmetric ( $N \geq 16-128$, depending on other conditions), the moments derived

892 above may potentially be used to construct approximate confidence intervals. If multivariate

893 normality (or any other explicit distribution) can be assumed, then it is straightforward to

894 obtain empirical distributions under appropriate conditions with Monte Carlo simulations.

895 Critical points of the null distributions and empirical power at $\alpha=0.05$ and 0.01 based on the

896 present simulations are presented in Table S1-S3 as a quick guide for sampling design.

897 Several limiting and approximate distributions have been proposed for related statistics (e.g.,

898 John, 1972; Nagao, 1973; Ledoit \& Wolf, 2002; Schott, 2005), which could be used for

899 simple null hypothesis testing with large $N$. Resampling-based tests are another potential

900 avenue of development. Applicability and performance of these alternative methods would

901 deserve further investigations.

902

\section{Acknowledgements}

904 The author would like to thank Carmelo Fruciano and Christian P. Klingenberg for

905 encouragements and constructive comments in an early stage of the study. This work was

906 partly supported by the Newton International Fellowships by the Royal Society 
907 (NIFIR1\180520) and the Overseas Research Fellowships by the Japan Society for the

908 Promotion of Science (202160529). The author declares no conflict of interest.

909

\section{References}

911 Adams DC. 2016. Evaluating modularity in morphometric data: challenges with the RV

912 coefficient and a new test measure. Methods in Ecology and Evolution 7: 565-572.

913 https://doi.org/10.1111/2041-210X.12511.

914 Adams DC, Collyer ML. 2018. Multivariate phylogenetic comparative methods: evaluations,

$915 \quad$ comparisons, and recommendations. Systematic Biology 67: 14-31.

916 https://doi.org/10.1093/sysbio/syx055.

917 Adams DC, Collyer ML. 2019a. Comparing the strength of modular signal, and evaluating

918 alternative modular hypotheses, using covariance ratio effect sizes with morphometric

919 data. Evolution 73: 2352-2367. https://doi.org/10.1111/evo.13867.

920 Adams DC, Collyer ML. 2019b. Phylogenetic comparative methods and the evolution of

921 multivariate phenotypes. Annual Review of Ecology, Evolution, and Systematics 50: 405-

922 425. https://doi.org/10.1146/annurev-ecolsys-110218-024555.

923 Adams DC, Felice RN. 2014. Assessing trait covariation and morphological integration on

924 phylogenies using evolutionary covariance matrices. PLoS ONE 9: e94335.

925 https://doi.org/10.1371/journal.pone.0094335.

926 Agrawal AF, Stinchcombe JR. 2009. How much do genetic covariances alter the rate of

927 adaptation? Proceedings of the Royal Society B: Biological Sciences 276: 1183-1191.

$928 \quad$ https://doi.org/10.1098/rspb.2008.1671.

929 Anderson TW. 1963. Asymptotic theory for principal component analysis. Annals of

$930 \quad$ Mathematical Statistics 34: 122-148. https://doi.org/10.1214/aoms/1177704248. 
931 Anderson TW. 2003. An Introduction to Multivariate Statistical Analysis, 3rd edn. Hoboken,

932 New Jersey: John Wiley \& Sons.

933 Arlegi M, Veschambre-Couture C, Gómez-Olivencia A. 2020. Evolutionary selection and

934 morphological integration in the vertebral column of modern humans. American Journal

935 of Physical Anthropology 171: 17-36. https://doi.org/10.1002/ajpa.23950.

936 Armbruster WS, Hansen TF, Pélabon C, Pérez-Barrales R, Maad J. 2009. The adaptive

937 accuracy of flowers: measurement and microevolutionary patterns. Annals of Botany 103:

938 1529-1545. https://doi.org/10.1093/aob/mcp095.

939 Armbruster WS, Pélabon C, Bolstad GH, Hansen TF. 2014. Integrated phenotypes:

940 understanding trait covariation in plants and animals. Philosophical Transactions of the

$941 \quad$ Royal Society B: Biological Sciences 369: 20130245.

942 https://doi.org/10.1098/rstb.2013.0245.

943 Bartoszek K, Pienaar J, Mostad P, Andersson S, Hansen TF. 2012. A phylogenetic

944 comparative method for studying multivariate adaptation. Journal of Theoretical Biology

945 314: 204-215. https://doi.org/10.1016/j.jtbi.2012.08.005.

946 Bastide P, Ané C, Robin S, Mariadassou M. 2018. Inference of adaptive shifts for

947 multivariate correlated traits. Systematic Biology 67: 662-680.

948 https://doi.org/10.1093/sysbio/syy005.

949 Björklund M. 2019. Be careful with your principal components. Evolution 73: 2151-2158.

950 https://doi.org/10.1111/evo.13835.

951 Blomberg SP, Garland T Jr, Ives AR. 2003. Testing for phylogenetic signal in comparative

952 data: behavioral traits are more labile. Evolution 57: 717-745.

953 https://doi.org/10.1111/j.0014-3820.2003.tb00285.x. 
954 Blows MW, McGuigan K. 2015. The distribution of genetic variance across phenotypic

$955 \quad$ space and the response to selection. Molecular Ecology 24: 2056-2072.

956 https://doi.org/10.1111/mec.13023.

957 Bolstad GH, Hansen TF, Pélabon C, Falahati-Anbaran M, Pérez-Baralles R,

958 Armbruster WS. 2014. Genetic constraints predict evolutionary divergence in

959 Dalechampia blossoms. Philosophical Transactions of the Royal Society B: Biological

$960 \quad$ Sciences 369: 20130255. https://doi.org/10.1098/rstb.2013.0255.

961 Brommer JE. 2014. Using average autonomy to test whether behavioral syndromes

962 constrain evolution. Behavioral Ecology and Sociobiology 68: 691-700.

963 https://doi.org/10.1007/s00265-014-1699-6.

964 Burnaby TP. 1966. Growth-invariant discriminant functions and generalized distances.

965 Biometrics 22: 96-110. https://doi.org/10.2307/2528217.

966 Caetano DS, Harmon LJ. 2019. Estimating correlated rates of trait evolution with

967 uncertainty. Systematic Biology 68: 412-429. https://doi.org/10.1093/sysbio/syy067.

968 Cane WP. 1993. The ontogeny of postcranial integration in the common tern, Sterna hirundo.

969 Evolution 47: 1138-1151. https://doi.org/10.1111/j.1558-5646.1993.tb02141.x.

970 Chenoweth SF, Rundle HD, Blows MW. 2010. The contribution of selection and genetic

971 constraints to phenotypic divergence. American Naturalist 175: 186-196.

972 https://doi.org/10.1086/649594.

973 Cheverud JM. 1982. Phenotypic, genetic, and environmental morphological integration in

974 the cranium. Evolution 36: 499-516. https://doi.org/10.1111/j.1558-5646.1982.tb05070.x.

975 Cheverud JM. 1988. A comparison of genetic and phenotypic correlations. Evolution 42:

976 958-968. https://doi.org/10.1111/j.1558-5646.1988.tb02514.x. 
977 Cheverud JM. 1996. Quantitative genetic analysis of cranial morphology in the cotton-top

978 (Saguinus oedipus) and saddle-back (S. fuscicollis) tamarins. Journal of Evolutionary

979 Biology 9: 5-42. https://doi.org/10.1046/j.1420-9101.1996.9010005.x.

980 Cheverud JM, Rutledge JJ, Atchley WR. 1983. Quantitative genetics of development:

981 genetic correlations among age-specific trait values and the evolution of ontogeny.

982 Evolution 37: 895-905. https://doi.org/10.1111/j.1558-5646.1983.tb05619.x.

983 Cheverud JM, Wagner GP, Dow MM. 1989. Methods for the comparative analysis of

$984 \quad$ variation patterns. Systematic Zoology 38: 201-213. https://doi.org/10.2307/2992282.

985 Clavel J, Aristide L, Morlon H. 2019. A penalized likelihood framework for high-

986 dimensional phylogenetic comparative methods and an application to New-World

987 monkeys brain evolution. Systematic Biology 68: 93-116.

988 https://doi.org/10.1093/sysbio/syy045.

989 Clavel J, Escarguel G, Merceron G. 2015. mvMORPH: an R package for fitting multivariate

$990 \quad$ evolutionary models to morphometric data. Methods in Ecology and Evolution 6: 1311-

991 1319. https://doi.org/10.1111/2041-210X.12420.

992 Clavel J, Morlon H. 2020. Reliable phylogenetic regressions for multivariate comparative

993 data: illustration with the MANOVA and application to the effect of diet and mandible

994 morphology in phyllostomid bats. Systematic Biology 69: 927-943.

995 https://doi.org/10.1093/sysbio/syaa010.

996 Constantine AG. 1963. Some non-central distribution problems in multivariate analysis.

997 Annals of Mathematical Statistics 34: 1270-1285.

998 https://doi.org/10.1214/aoms/1177703863.

999 Cramer JS. 1987. Mean and variance of $R^{2}$ in small and moderate samples. Journal of

$1000 \quad$ Econometrics 35: 253-266. https://doi.org/10.1016/0304-4076(87)90027-3. 
1001 Darroch JN, Mosimann JE. 1985. Canonical and principal components of shape.

$1002 \quad$ Biometrika 72: 241-252. https://doi.org/10.1093/biomet/72.2.241.

1003 Davies PI, Higham NJ. 2000. Numerically stable generation of correlation matrices and

1004 their factors. BIT Numerical Mathematics 40: 640-651.

1005 https://doi.org/10.1023/A:1022384216930.

1006 Dochtermann NA. 2011. Testing Cheverud's conjecture for behavioral correlations and

1007 behavioral syndromes. Evolution 65: 1814-1820. https://doi.org/10.1111/j.1558-

$1008 \quad$ 5646.2011.01264.x.

1009 Durand J-L, Le Roux B. 2017. Linkage index of variables and its relationship with variance

1010 of eigenvalues in PCA and MCA. Statistica Applicata - Italian Journal of Applied

1011 Statistics 29: 123-135. https://doi.org/10.26398/IJAS.0029-006.

1012 Eddelbuettel D, Balamuta JJ. 2018. Extending $R$ with C++: a brief introduction to Rcpp.

1013 American Statistician 72: 28-36. https://doi.org/10.1080/00031305.2017.1375990.

1014 Felice RN, Randau M, Goswami A. 2018. A fly in a tube: macroevolutionary expectations

1015 for integrated phenotypes. Evolution 72: 2580-2594. https://doi.org/10.1111/evo.13608.

1016 Felsenstein J. 1988. Phylogenies and quantitative characters. Annual Review of Ecology and

1017 Systematics 19: 445-471. https://doi.org/10.1146/annurev.es.19.110188.002305.

1018 Fornoni J, Ordano M, Boege K, Domínguez CA. 2009. Phenotypic integration: between

1019 zero and how much is too much. New Phytologist 183: 248-250.

$1020 \quad$ https://doi.org/10.1111/j.1469-8137.2009.02911.x.

1021 Freckleton RP, Harvey PH, Pagel M. 2002. Phylogenetic analysis of comparative data: a

1022 test and review of evidence. American Naturalist 160: 712-726.

1023 https://doi.org/10.1086/343873.

1024 Garamszegi LZ, Mundry R. 2014. Multimodel-inference in comparative analyses. In:

1025 Garamszegi LZ, ed. Modern Phylogenetic Comparative Methods and Their Applications 
1026

1027

1028

1029

1030

1031

1032

1033

1034

1035

1036

1037

1038

1039

1040

1041

1042

1043

1044

1045

1046

1047

1048

in Evolutionary Biology: Concepts and Practice. Berlin: Springer, 305-331. https://doi.org/10.1007/978-3-662-43550-2_12.

Ghosh BK. 1966. Asymptotic expansions for the moments of the distribution of correlation coefficient. Biometrika 53: 258-262. https://doi.org/10.2307/2334076.

Girshick MA. 1939. On the sampling theory of roots of determinantal equations. Annals of Mathematical Statistics 10: 203-224. https://doi.org/10.1214/aoms/1177732180.

Gleason TC, Staelin R. 1975. A proposal for handling missing data. Psychometrika 40: 229252. https://doi.org/10.1007/BF02291569.

Goswami A. 2006. Morphological integration in the carnivoran skull. Evolution 60: 169-183. https://doi.org/10.1111/j.0014-3820.2006.tb01091.x.

Goswami A, Binder WJ, Mearchen J, O'Keefe FR. 2015. The fossil record of phenotypic integration and modularity: a deep-time perspective on developmental and evolutionary dynamics. Proceedings of the National Academy of Sciences of the United States of America 112: 4891-4896. https://doi.org/10.1073/pnas.1403667112.

Goswami A, Finnarelli JA. 2016. EMMLi: a maximum likelihood approach to the analysis of modularity. Evolution 70: 1622-1637. https://doi.org/10.1111/evo.12956.

Goswami A, Polly PD. 2010. Methods for studying morphological integration and modularity. In: Alroy J, Hunt G, eds. Quantitative Methods in Paleobiology. Paleontological Society Papers 16: 213-243. https://doi.org/10.1017/S1089332600001881.

Grabowski M, Porto A. 2017. How many more? Sample size determination in studies of morphological integration and evolvability. Methods in Ecology and Evolution 8: 592603. https://doi.org/10.1111/2041-210X.12674. 
1049 Grafen A. 1989. The phylogenetic regression. Philosophical Transactions of the Royal

$1050 \quad$ Society of London B: Biological Sciences 326: 119-157.

1051 https://doi.org/10.1098/rstb.1989.0106.

1052 Gupta AK, Nagar DK. 1999. Matrix Variate Distributions. Boca Raton, Florida: Chapman $1053 \quad \&$ Hall/CRC.

1054 Haber A. 2011. A comparative analysis of integration indices. Evolutionary Biology 38:

1055 476-488. https://doi.org/10.1007/s11692-011-9137-4.

1056 Haber A. 2015. The evolution of morphological integration in the ruminant skull.

1057 Evolutionary Biology 42: 99-114. https://doi.org/10.1007/s11692-014-9302-7.

1058 Haber A. 2016. Phenotypic covariation and morphological diversification in the ruminant

1059 skull. American Naturalist 187: 576-591. https://doi.org/10.1086/685811.

1060 Haber A, Dworkin I. 2017. Disintegrating the fly: a mutational perspective on phenotypic

1061 integration and covariation. Evolution 71: 66-80. https://doi.org/10.1111/evo.13100.

1062 Hallgrímsson B, Jamniczky H, Young NM, Rolian C, Parsons TE, Boughner JC,

1063 Marcucio RS. 2009. Deciphering the palimpsest: studying the relationships between

1064 morphological integration and phenotypic covariation. Evolutionary Biology 36: 355-376.

1065 https://doi.org/10.1007/s11692-009-9076-5.

1066 Hankin RKS. 2015. Numerical evaluation of the Gauss hypergeometric function with the

1067 hypergeo package. The R Journal 7: 81-88. https://doi.org/10.32614/RJ-2015-022.

1068 Hansen TF, Houle D. 2008. Measuring and comparing evolvability and constraint in

1069 multivariate characters. Journal of Evolutionary Biology 21: 1201-1219.

$1070 \quad$ https://doi.org/10.1111/j.1420-9101.2008.01573.x.

1071 Hansen TF, Martins E. 1996. Translating between microevolutionary process and

1072 macroevolutionary patterns: the correlation structure of interspecific data. Evolution 50:

1073 1404-1417. https://doi.org/10.1111/j.1558-5646.1996.tb03914.x. 
1074 Hansen TF, Solvin TM, Pavlicev M. 2019. Predicting evolutionary potential: a numerical

1075 test of evolvability measures. Evolution 73: 689-703. https://doi.org/10.1111/evo.13705.

1076 Harder LD. 2009. Questions about floral (dis)integration. New Phytologist 183: 247-248.

1077 https://doi.org/10.1111/j.1469-8137.2009.02881.x.

1078 Hereford J, Hansen TF, Houle D. 2004. Comparing strengths of directional selection: how

1079 strong is strong? Evolution 58: 2133-2143. https://doi.org/10.1111/j.0014-

$1080 \quad$ 3820.2004.tb01592.x.

1081 Ho LST, Ané C. 2014. Intrinsic inference difficulties for trait evolution with Ornstein-

1082 Uhlenbeck models. Methods in Ecology and Evolution 5: 1133-1146.

1083 https://doi.org/10.1111/2041-210X.12285.

1084 Houle D. 1992. Comparing evolvability and variability of quantitative traits. Genetics 130:

1085 195-204. https://doi.org/10.1093/genetics/130.1.195.

1086 Huelsenbeck JP, Rannala B. 2003. Detecting correlation between characters in a

1087 comparative analysis with uncertain phylogeny. Evolution 57: 1237-1247.

$1088 \quad$ https://doi.org/10.1111/j.0014-3820.2003.tb00332.x.

1089 John S. 1971. Some optimal multivariate tests. Biometrika 58: 123-127.

1090 https://doi.org/10.1093/biomet/58.1.123.

1091 John S. 1972. The distribution of a statistic used for testing sphericity of normal distributions.

1092 Biometrika 59: 169-173. https://doi.org/10.1093/biomet/59.1.169.

1093 Johnstone IM. 2007. High dimensional statistical inferences and random matrices. In: Sanz-

1094 Solé M, Soria J, Varona JL, Verdera J, eds. Proceedings of the International Congress of

1095 Mathematicians, Madrid, August 22-30, 2006, Vol. 1. Zürich: European Mathematical

$1096 \quad$ Society, 307-333.

1097 Johnstone IM, Paul D. 2018. PCA in high dimensions: an orientation. Proceedings of the

1098 IEEE 106: 1277-1292. https://doi.org/10.1109/JPROC.2018.2846730. 
1099 Jolicoeur P. 1963. The multivariate generalization of the allometry equation. Biometrics 19:

1100 497-499. https://doi.org/10.2307/2527939.

1101 Jolliffe IT. 2002. Principal Component Analysis, 2nd edn. New York: Springer.

1102 Jung H, Conaway MA, von Cramon-Taubadel N. 2020. Examination of sample size

1103 determination in integration studies based on the integration coefficient of variation (ICV).

1104 Evolutionary Biology 47: 293-307. https://doi.org/10.1007/s11692-020-09514-w.

1105 Kirkpatrick M. 2009. Patterns of quantitative genetic variation in multiple dimensions.

1106 Genetica 136: 271-284. https://doi.org/10.1007/s10709-008-9302-6.

1107 Klingenberg CP. 1996. Multivariate allometry. In: Marcus LF, ed. Advances in

1108 Morphometrics. New York: Plenum Press, 23-49. https://doi.org/10.1007/978-1-4757-

1109 9083-2_3.

1110 Klingenberg CP. 2014. Studying morphological integration and modularity at multiple

1111 levels: concepts and analysis. Philosophical Transactions of the Royal Society B:

1112 Biological Sciences 369: 20130249. https://doi.org/10.1098/rstb.2013.0249.

1113 Klingenberg CP. 2016. Size, shape, and form: concepts of allometry in geometric

1114 morphometrics. Development Genes and Evolution 226: 113-137.

1115 https://doi.org/10.1007/s00427-016-0539-2.

1116 Klingenberg CP. 2020. Walking on Kendall's shape space: understanding shape spaces and

1117 their coordinate systems. Evolutionary Biology 47: 334-352.

$1118 \quad$ https://doi.org/10.1007/s11692-020-09513-x.

1119 Klingenberg CP. 2021. How exactly did the nose get that long? A critical rethinking of the

$1120 \quad$ Pinocchio effect and how shape changes relate to landmarks. Evolutionary Biology 48:

1121 115-127. https://doi.org/10.1007/s11692-020-09520-y.

1122 Klingenberg CP, Duttke S, Whelan S, Kim M. 2012. Developmental plasticity,

1123 morphological variation and evolvability: a multilevel analysis of morphometric 
1124 integration in the shape of compound leaves. Journal of Evolutionary Biology 25: 115-

1125 129. https://doi.org/10.1111/j.1420-9101.2011.02410.x.

1126 Konishi S. 1979. Asymptotic expansions for the distributions of statistics based on the

1127 sample correlation matrix in principal component analysis. Hiroshima Mathematical

1128 Journal 9: 647-700. https://doi.org/10.32917/hmj/1206134750.

1129 Lande R. 1979. Quantitative genetic analysis of multivariate evolution, applied to brain:body

$1130 \quad$ size allometry. Evolution 33: 402-416. https://doi.org/10.1111/j.1558-

$1131 \quad$ 5646.1979.tb04694.x.

1132 Lande R, Arnold SJ. 1983. The measurement of selection on correlated characters.

1133 Evolution 37: 1210-1226. https://doi.org/10.1111/j.1558-5646.1983.tb00236.x.

1134 Lawley DN. 1956. Tests of significance for the latent roots of covariance and correlation

1135 matrices. Biometrika 43: 128-136. https://doi.org/10.2307/2333586.

1136 Ledoit O, Wolf M. 2002. Some hypothesis tests for the covariance matrix when the

1137 dimension is large compared to the sample size. Annals of Statistics 30: 1081-1102.

1138 https://doi.org/10.1214/aos/1031689018.

1139 Lynch M, Walsh B. 1998. Genetics and Analysis of Quantitative Traits. Sunderland,

$1140 \quad$ Massachusetts: Sinauer Associates.

1141 Machado FA, Hubbe A, Melo D, Porto A, Marroig G. 2019. Measuring the magnitude of

1142 morphological integration: the effect of differences in morphometric representations and

1143 the inclusion of size. Evolution 73: 2518-2528. https://doi.org/10.1111/evo.13864.

1144 Manceau M, Lambert A, Morlon H. 2017. A unifying comparative phylogenetic

1145 framework including traits coevolving across interacting lineages. Systematic Biology 66:

1146 551-568. https://doi.org/10.1093/sysbio/syw115. 
1147 Marcus LF, Hingst-Zaher E, Zaher H. 2000. Application of landmark morphometrics to

1148 skulls representing the orders of living mammals. Hystrix 11: 27-47.

1149 https://doi.org/10.4404/hystrix-11.1-4135.

1150 Marroig G, Melo DAR, Garcia G. 2012. Modularity, noise, and natural selection. Evolution $1151 \quad$ 66: $1506-1524$. https://doi.org/10.1111/j.1558-5646.2011.01555.x.

1152 Marroig G, Shirai LT, Porto A, de Oliveira FB, De Conto V. 2009. The evolution of 1153 modularity in the mammalian skull II: evolutionary consequences. Evolutionary Biology $1154 \quad 36:$ 136-148. https://doi.org/10.1007/s11692-009-9051-1.

1155 Martins E, Hansen TF. 1997. Phylogenies and the comparative method: a general approach 1156 to incorporating phylogenetic information into the analysis of interspecific data.

1157 American Naturalist 149: 646-667. https://doi.org/10.1086/286013.

1158 Meyer K, Kirkpatrick M. 2005. Restricted maximum likelihood estimation of genetic 1159 principal components and smoothed covariance matrices. Genetics Selection Evolution $1160 \quad 37:$ 1-30. https://doi.org/10.1051/gse:2004034.

1161 Mitov V, Bartoszek K, Asimomitis G, Stadler T. 2020. Fast likelihood calculation for 1162 multivariate Gaussian phylogenetic models with shifts. Theoretical Population Biology 1163 131: 66-78. https://doi.org/10.1016/j.tpb.2019.11.005.

1164 Mitov V, Bartoszek K, Stadler T. 2019. Automatic generation of evolutionary hypotheses 1165 using mixed Gaussian phylogenetic models. Proceedings of the National Academy of $1166 \quad$ Sciences of the United States of America 116: 16921-16926.

1167 https://doi.org/10.1073/pnas.1813823116.

1168 Mitteroecker P, Gunz P, Neubauer S, Müller G. 2012. How to explore morphological 1169 integration in human evolution and development? Evolutionary Biology 39: 536-553. 1170 https://doi.org/10.1007/s11692-012-9178-3. 
1171 Mosimann JE. 1970. Size allometry: size and shape variables with characterizations of the

1172 lognormal and generalized gamma distributions. Journal of the American Statistical

1173 Association 65: 930-945. https://doi.org/10.1080/01621459.1970.10481136.

1174 Mosimann JE, James FC. 1979. New statistical methods for allometry with application to

1175 Florida red-winged blackbirds. Evolution 33: 444-459. https://doi.org/10.1111/j.1558-

$1176 \quad$ 5646.1979.tb04697.x.

1177 Muirhead RJ. 1982. Aspects of Multivariate Statistical Theory. Hoboken, New Jersey: John $1178 \quad$ Wiley \& Sons.

1179 Nagao H. 1973. On some test criteria for covariance matrix. Annals of Statistics 1: 700-709.

1180 https://doi.org/10.1214/aos/1176342464.

1181 Nakagawa S, de Villemereuil P. 2019. A general method for simultaneously accounting for

1182 phylogenetic and species sampling uncertainty via Rubin's rules in comparative analysis.

$1183 \quad$ Systematic Biology 68: 632-641. https://doi.org/10.1093/sysbio/syy089.

1184 Olkin I, Siotani M. 1976. Asymptotic distribution of functions of a correlation matrix. In:

1185 Editorial Committee for Publication of Essays in Probability and Statistics, eds. Essays in

1186 Probability and Statistics in Honor of Professor Junjiro Ogawa. Tokyo: Shinko Tsusho,

$1187 \quad 235-251$.

1188 Olson EC, Miller RL. 1958. Morphological Integration. [Chicago]: University of Chicago

1189 Press.

1190 Ordano M, Fornoni J, Boege K, Domínguez CA. 2008. The adaptive value of phenotypic

1191 floral integration. New Phytologist 179: 1183-1192. https://doi.org/10.1111/j.1469-

$1192 \quad$ 8137.2008.02523.x.

1193 Pan W, Frank KA. 2004. An approximation to the distribution of the product of two

1194 dependent correlation coefficients. Journal of Statistical Computation and Simulation 74:

1195 419-443. https://doi.org/10.1080/00949650310001596822. 
1196 Pavlicev M, Cheverud JM, Wagner GP. 2009. Measuring morphological integration using

1197 eigenvalue variance. Evolutionary Biology 36: 157-170. https://doi.org/10.1007/s11692-

$1198 \quad 008-9042-7$.

1199 Pitchers W, Wolf JB, Tregenza T, Hunt J, Dworkin I. 2014. Evolutionary rates for

1200 multivariate traits: the role of selection and genetic variation. Philosophical Transactions

1201 of the Royal Society B: Biological Sciences 369: 20130252.

1202 https://doi.org/10.1098/rstb.2013.0252.

1203 Porto A, de Oliveira FB, Shirai LT, De Conto V, Marroig G. 2009. The evolution of

1204 modularity in the mammalian skull I: morphological integration patterns and magnitudes.

1205 Evolutionary Biology 36: 118-135. https://doi.org/10.1007/s11692-008-9038-3.

1206 R Core Team. 2019. $R$ : a language and environment for statistical computing, Version 3.5.3.

1207 Vienna: R Foundation for Statistical Computing. https://www.R-project.org/.

1208 Renaud S, Auffray J-C. 2013. The direction of main phenotypic variance as a channel to

1209 morphological evolution: case studies in murine rodents. Hystrix 24: 85-93.

$1210 \quad$ https://doi.org/10.4404/hystrix-24.1-6296.

1211 Revell LJ. 2009. Size-correction and principal components for interspecific comparative

1212 studies. Evolution 63: 3258-3268. https://doi.org/10.1111/j.1558-5646.2009.00804.x.

1213 Revell LJ, Collar DC. 2009. Phylogenetic analysis of the evolutionary correlation using

1214 likelihood. Evolution 63: 1090-1100. https://doi.org/10.1111/j.1558-5646.2009.00616.x.

1215 Revell LJ, González-Valenzuela LE, Alfonso A, Castellanos-García LA, Guarnizo CE,

1216 Crawford AJ. 2018. Comparing evolutionary rates between trees, clades and traits.

1217 Methods in Ecology and Evolution 9: 994-1005. https://doi.org/10.1111/2041-

$1218 \quad$ 210X.12977. 
1219 Revell LJ, Harmon LJ. 2008. Testing quantitative genetic hypotheses about the

1220 evolutionary rate matrix for continuous characters. Evolutionary Ecology Research 10:

$1221 \quad 311-331$.

1222 Revell LJ, Harrison AS. 2008. PCCA: a program for phylogenetic canonical correlation

1223 analysis. Bioinformatics 24: 1018-1020. https://doi.org/10.1093/bioinformatics/btn065.

1224 Roff DA. 1995. The estimation of genetic correlations from phenotypic correlations: a test of

1225 Cheverud's conjecture. Heredity 74: 481-490. https://doi.org/10.1038/hdy.1995.68.

1226 Rohlf FJ. 1999. Shape statistics: Procrustes superimpositions and tangent spaces. Journal of

1227 Classification 16: 197-223. https://doi.org/10.1007/s003579900054.

1228 Rohlf FJ. 2001. Comparative methods for the analysis of continuous variables: geometric

1229 interpretations. Evolution 55: 2143-2160. https://doi.org/10.1111/j.0014-

$1230 \quad$ 3820.2001.tb00731.x.

1231 Rohlf FJ. 2006. A comment on phylogenetic correction. Evolution 60: 1509-1515.

1232 https://doi.org/10.1111/j.0014-3820.2006.tb01229.x.

1233 Rohlf FJ, Bookstein FL. 2003. Computing the uniform component of shape variation.

1234 Systematic Biology 52: 66-69. https://doi.org/10.1080/10635150390132759.

1235 Rohlf FJ, Slice D. 1990. Extensions of the Procrustes method for the optimal

1236 superimposition of landmarks. Systematic Zoology 39: 40-59.

$1237 \quad$ https://doi.org/10.2307/2992207.

1238 Schluter D. 1996. Adaptive radiation along genetic lines of least resistance. Evolution 50:

1239 1766-1774. https://doi.org/10.1111/j.1558-5646.1996.tb03563.x.

1240 Schott JR. 2005. Testing for complete independence in high dimensions. Biometrika 92:

1241 951-956. https://doi.org/10.1093/biomet/92.4.951.

1242 Shirai LT, Marroig G. 2010. Skull modularity in Neotropical marsupials and monkeys: size

1243 variation and evolutionary constraint and flexibility. Journal of Experimental Zoology, 
1244 Part B: Molecular and Developmental Evolution 314B: 663-683.

1245 https://doi.org/10.1002/jez.b.21367.

1246 Slice DE. 2001. Landmark coordinates aligned by Procrustes analysis do not lie in Kendall's

1247 shape space. Systematic Biology 50: 141-149. https://doi.org/10.1080/10635150119110.

1248 Sodini SM, Kemper KE, Wray NR, Trzaskowski M. 2018. Comparison of genotypic and

1249 phenotypic correlations: Cheverud's conjecture in humans. Genetics 209: 941-948.

1250 https://doi.org/10.1534/genetics.117.300630.

1251 Soper HE, Young AW, Cave BM, Lee A, Pearson K. 1917. On the distribution of the

1252 correlation coefficient in small samples. Appendix II to the papers of "Student" and R. A.

1253 Fisher. Biometrika 11: 328-413. https://doi.org/10.1093/biomet/11.4.328.

1254 Srivastava MS, Khatri CG. 1979. An Introduction to Multivariate Statistics. New York:

1255 North Holland.

1256 Srivastava MS, Yanagihara H. 2010. Testing the equality of several covariance matrices

1257 with fewer observations than the dimension. Journal of Multivariate Analysis 101: 1319-

1258 1329. https://doi.org/10.1016/j.jmva.2009.12.010.

1259 Steppan SJ, Phillips PC, Houle D. 2002. Comparative quantitative genetics: evolution of

1260 the G matrix. Trends in Ecology and Evolution 17: 320-327.

$1261 \quad$ https://doi.org/10.1016/S0169-5347(02)02505-3.

1262 Stuart A, Ord JK. 1994. Kendall's Advanced Theory of Statistics, 6th edn, Vol. 1. London:

1263 Hodder Education [Reprinted in 2004 by John Wiley \& Sons, Chichester].

1264 Sugiura N. 1972. Locally best invariant test for sphericity and the limiting distributions.

1265 Annals of Mathematical Statistics 43: 1312-1316.

1266 https://doi.org/10.1214/aoms/1177692481.

1267 Symonds MRE, Blomberg SP. 2014. A primer on phylogenetic generalized least squares.

1268 In: Garamszegi LZ, ed. Modern Phylogenetic Comparative Methods and Their 
Application in Evolutionary Biology: Concepts and Practice. Berlin: Springer, 105-130. https://doi.org/10.1007/978-3-662-43550-2_5.

1271 Sztepanacz JL, Blows MW. 2017. Accounting for sampling error in genetic eigenvalues

1272 using random matrix theory. Genetics 206: 1271-1284.

1273 https://doi.org/10.1534/genetics.116.198606.

1274 Torices R, Méndez M. 2014. Resource allocation to inflorescence components is highly

1275 integrated despite differences between allocation currencies and sites. International

$1276 \quad$ Journal of Plant Sciences 175: 713-723. https://doi.org/10.1086/676622.

1277 Torices R, Muñoz-Pajares AJ. 2015. PHENIX: an R package to estimate a size-controlled

1278 phenotypic integration index. Applications in Plant Sciences 3: 1400104.

1279 https://doi.org/10.3732/apps.1400104.

1280 Uyeda JC, Harmon LJ. 2014. A novel Bayesian method for inferring and interpreting the 1281 dynamics of adaptive landscapes from phylogenetic comparative data. Systematic Biology

$1282 \quad$ 63: 902-918. https://doi.org/10.1093/sysbio/syu057.

1283 Van Valen L. 1974. Multivariate structural statistics in natural history. Journal of

1284 Theoretical Biology 45: 235-247. https://doi.org/10.1016/0022-5193(74)90053-8.

1285 Van Valen L. 2005. The statistics of variation. In: Hallgrímsson B, Hall BK, eds. Variation.

1286 Amsterdam: Elsevier, 29-47. https://doi.org/10.1016/B978-012088777-4/50005-3.

1287 de Waal DJ, Nel DG. 1973. On some expectations with respect to Wishart matrices. South $1288 \quad$ African Statistical Journal 7: 61-67.

1289 Wagner GP. 1984. On the eigenvalue distribution of genetic and phenotypic dispersion

1290 matrices: evidence for a nonrandom organization of quantitative character variation.

1291 Journal of Mathematical Biology 21: 77-95. https://doi.org/10.1007/BF00275224. 
1292 Walker JA. 2000. Ability of geometric morphometric methods to estimate a known

1293 covariance matrix. Systematic Biology 49: 686-696.

1294 https://doi.org/10.1080/106351500750049770.

1295 Waller NG. 2020. Generating correlation matrices with specified eigenvalues using the

1296 method of alternating projections. American Statistician 74: 21-28.

1297 https://doi.org/10.1080/00031305.2017.1401960.

1298 Watanabe J. 2018. Clade-specific evolutionary diversification along ontogenetic major axes

1299 in avian limb skeleton. Evolution 72: 2632-2652. https://doi.org/10.1111/evo.13627.

\section{Appendix A}

1302 In this part, relationships between eigenvalue dispersion indices and individual eigenvalues

1303 are derived under certain restrictive conditions, in order to facilitate interpretation and to

1304 clarify algorithms used in simulations. For simplicity, it is assumed $\bar{\lambda}=1$ in the following

1305 discussions; general cases easily follow by scaling.

1306 Let us first consider the simple conditions where the first $q(<p)$ population

1307 eigenvalues are equally large and the rest $p-q$ eigenvalues are equally small: $\lambda_{1}=\cdots=$

$1308 \lambda_{q} \geq \lambda_{q+1}=\cdots=\lambda_{p}$ (" $q$-large $\lambda$ conditions" in simulations). By noting $\sum \lambda_{i}=q \lambda_{1}+$

$1309(p-q) \lambda_{p}=p$, it is seen that

$$
V_{\text {rel }}(\Sigma)=\frac{\sum_{i=1}^{p}\left(\lambda_{i}-1\right)^{2}}{p(p-1)}=\frac{(p-q)}{q(p-1)}\left(1-\lambda_{p}\right)^{2},
$$

1311 and hence

$$
\begin{aligned}
& \lambda_{1}=1+\sqrt{\frac{(p-1)(p-q)}{q} V_{\text {rel }}}, \\
& \lambda_{p}=1-\sqrt{\frac{q(p-1)}{p-q} V_{\text {rel }} .}
\end{aligned}
$$


1313 By noting the constraint $0 \leq \lambda_{p} \leq 1$, an upper limit of $V_{\text {rel }}$ can be seen from equation A1:

$$
V_{\text {rel }}(\Sigma) \leq \frac{(p-q)}{q(p-1)}=\frac{1}{q}-\frac{q-1}{q(p-1)} .
$$

1315 It is then obvious that, under these constraints, a value of $V_{\text {rel }}$ greater than 0.5 cannot happen

1316 when $q>1$; that is, such a large value implies the dominance of a single component of

1317 variance. The same arguments equally apply to correlation matrices.

1318 When $q=1$ for the correlation matrix, $V_{\text {rel }}(\mathbf{P})$ completely specifies the magnitude of

1319 correlation in every pair of variables. This point can be seen from the definition of

1320 eigendecomposition:

$$
\begin{aligned}
\rho_{i j} & =\sum_{k=1}^{p} \lambda_{k} \mathrm{v}_{i k} \mathrm{v}_{j k} \\
& =\left(\lambda_{1}-\lambda_{p}\right) \mathrm{v}_{i 1} \mathrm{v}_{j 1}+\lambda_{p} \sum_{k=1}^{p} \mathrm{v}_{i k} \mathrm{v}_{j k},
\end{aligned}
$$

where $\mathrm{v}_{i j}$ is the $(i, j)$-th element of the eigenvector matrix. By remembering $\sum_{k=1}^{p} \mathrm{v}_{i k} \mathrm{v}_{j k}=$

$\delta_{i j}$, the Kronecker delta, and noting equation A2 with $q=1$,

$$
1=\rho_{i i}=\left(\lambda_{1}-\lambda_{p}\right) v_{i 1}^{2}+\lambda_{p}=\left(p v_{i 1}^{2}-1\right) \sqrt{V_{\text {rel }}}+1
$$

1325 therefore $\mathrm{v}_{i 1}^{2}=p^{-1 / 2}$ for any $i$ (that is, the coefficients of the first eigenvector are equal in 1326 magnitude). Finally, we have

$$
\rho_{i j}^{2}=\left(\lambda_{1}-\lambda_{p}\right)^{2} v_{i 1}^{2} v_{j 1}^{2}=V_{\text {rel }}
$$

1328 for any combination of $i$ and $j(i \neq j)$; the magnitude of correlation is identical across all

1329 pairs. Taken differently, $\lambda_{2}=\cdots=\lambda_{p}=|\rho|$. These relationships have previously been noted

1330 by Anderson (1963) and Pavlicev et al. (2009).

1331 The population eigenvalues of the linearly and quadratically decreasing $\lambda$ conditions

1332 used in simulations are defined as $\lambda_{i}=(p-i+1) \lambda_{p}$ and $\lambda_{i}=(p-i+1)^{2} \lambda_{p}(i=$ 
$13331,2, \ldots, p)$ for linearly and quadratically decreasing conditions, respectively. Under the

1334 assumption of a constant average eigenvalue, simple algebra yields the actual values of $\lambda_{p}$

1335 and $V_{\text {rel }}(\Sigma)$ as functions of $p$. The latter equals $1 / 3(p+1)$ and $(8 p+11) / 5(p+1)(2 p+$

1336 1) for the linearly and quadratically decreasing $\lambda$ conditions, respectively.

\section{Appendix B}

1339 In this part, the first two moments of $V(\mathbf{S})$ and $V_{\text {rel }}(\mathbf{S})$ under the arbitrary $\boldsymbol{\Sigma}$ are derived,

1340 assuming multivariate normality. Derivation of the moments of the latter requires evaluation

1341 of moments of the ratio $\sum l_{i}^{2} /\left(\sum l_{i}\right)^{2}=\operatorname{tr}\left(\mathbf{A}^{2}\right) /(\operatorname{tr} \mathbf{A})^{2}$, which are not guaranteed to coincide

1342 with the ratios of moments except under the null hypothesis. Here we utilize the

1343 approximation based on the delta method (eq. 32). In turn, we need $\mathrm{E}\left[\operatorname{tr}\left(\mathbf{A}^{2}\right)\right], \mathrm{E}\left[(\operatorname{tr} \mathbf{A})^{2}\right]$,

$1344 \operatorname{Var}\left[\operatorname{tr}\left(\mathbf{A}^{2}\right)\right], \operatorname{Var}\left[(\operatorname{tr} \mathbf{A})^{2}\right]$, and $\operatorname{Cov}\left[\operatorname{tr}\left(\mathbf{A}^{2}\right),(\operatorname{tr} \mathbf{A})^{2}\right]$.

1345 We will follow Srivastava \& Yanagihara's (2010) approach to obtain these moments.

1346 As in the text, let the $n \times p$ matrix $\mathbf{Z}$ be $\left(\mathbf{z}_{1}, \mathbf{z}_{2}, \ldots, \mathbf{z}_{n}\right)^{T}$, where $\mathbf{z}_{i} \sim N_{p}\left(\mathbf{0}_{p}, \mathbf{\Sigma}\right)$ for $i=$

$1347 \quad 1,2, \ldots, n$. Consider the cross-product matrix

1348

$$
\mathbf{A}=\mathbf{Z}^{T} \mathbf{Z}
$$

such that $\mathbf{A} \sim W_{p}(\boldsymbol{\Sigma}, n)$. Let the spectral decomposition of $\boldsymbol{\Sigma}$ :

$$
\boldsymbol{\Sigma}=\mathbf{Y} \boldsymbol{\Lambda} \mathbf{Y}^{T}
$$

1351 with the orthogonal matrix of eigenvectors $\mathbf{Y}$ and the diagonal matrix of eigenvalues $\boldsymbol{\Lambda}$. Let

1352 the $n \times p$ matrix $\mathbf{J}$ be $\left(\mathbf{j}_{1}, \mathbf{j}_{2}, \ldots, \mathbf{j}_{n}\right)^{T}$, where $\mathbf{j}_{i}$ are i.i.d. $N_{p}\left(\mathbf{0}, \mathbf{I}_{p}\right)$, such that $\mathbf{Z}=\mathbf{J} \mathbf{\Sigma}^{1 / 2}$ with

$1353 \boldsymbol{\Sigma}^{1 / 2}=\mathbf{Y} \boldsymbol{\Lambda}^{1 / 2} \boldsymbol{Y}^{T}$. Then, it is possible to write

$$
\mathbf{A}=\boldsymbol{\Sigma}^{1 / 2} \mathbf{J}^{T} \mathbf{J} \boldsymbol{\Sigma}^{1 / 2}=\mathbf{Y} \boldsymbol{\Lambda}^{1 / 2} \mathbf{Y}^{T} \mathbf{J}^{T} \mathbf{J} \mathbf{Y} \boldsymbol{\Lambda}^{1 / 2} \mathbf{Y}^{T}=\mathbf{Y} \boldsymbol{\Lambda}^{1 / 2} \mathbf{V}^{T} \mathbf{V} \boldsymbol{\Lambda}^{1 / 2} \mathbf{Y}^{T}
$$

1355 where $\mathbf{V}=\mathbf{J} \mathbf{Y}=\left(\mathbf{v}_{1}, \mathbf{v}_{2}, \ldots, \mathbf{v}_{p}\right)$ with $\mathbf{v}_{i}$ being i.i.d. $N_{n}\left(\mathbf{0}, \mathbf{I}_{n}\right)$. Furthermore, let $w_{i j}=\mathbf{v}_{i}^{T} \mathbf{v}_{j}$,

1356 such that $w_{i i}$ are i.i.d. chi-square variables with $n$ degrees of freedom $\chi_{n}^{2}$. Obviously $w_{i j}=$ 
$w_{j i}$. Note that

$$
\operatorname{tr} \mathbf{A}=\operatorname{tr}\left(\mathbf{Y} \boldsymbol{\Lambda}^{1 / 2} \mathbf{V}^{T} \mathbf{V} \boldsymbol{\Lambda}^{1 / 2} \mathbf{Y}^{T}\right)=\operatorname{tr}\left(\boldsymbol{\Lambda} \mathbf{V}^{T} \mathbf{V}\right)=\sum_{i=1}^{n} \lambda_{i} w_{i i}
$$

From well-known results on normal and chi-square moments, we have the following:

$$
\begin{aligned}
\mathrm{E}\left(w_{i i}^{r}\right) & =n(n+2) \ldots(n+2 r-2), \quad r=1,2, \ldots, \\
\mathrm{E}\left(w_{i i}^{r} w_{i j}^{2}\right) & =\mathrm{E}\left[\operatorname{tr}\left(w_{i i}^{r} \mathbf{v}_{i} \mathbf{v}_{i}^{T} \mathbf{v}_{j} \mathbf{v}_{j}^{T}\right)\right] \\
& =\operatorname{tr}\left[\mathrm{E}\left(w_{i i}^{r} \mathbf{v}_{i} \mathbf{v}_{i}^{T}\right) \mathrm{E}\left(\mathbf{v}_{j} \mathbf{v}_{j}^{T}\right)\right] \\
& =\operatorname{tr}\left[\mathrm{E}\left(w_{i i}^{r} \mathbf{v}_{i} \mathbf{v}_{i}^{T}\right) \mathbf{I}_{n}\right] \\
& =n(n+2) \ldots(n+2 r), \quad i \neq j, \quad r=0,1, \ldots, \\
\mathrm{E}\left(w_{i i} w_{j j} w_{i j}^{2}\right) & =\mathrm{E}\left[\operatorname{tr}\left(w_{i i} \mathbf{v}_{i} \mathbf{v}_{i}^{T} w_{j j} \mathbf{v}_{j} \mathbf{v}_{j}^{T}\right)\right] \\
& =\sum_{\alpha, \beta, \gamma, \delta}^{n} \mathrm{E}\left(v_{i \alpha}^{2} v_{i \gamma} v_{i \delta}\right) \mathrm{E}\left(v_{j \beta}^{2} v_{j \delta} v_{j \gamma}\right) \\
& =9 n+6 n(n-1)+n(n-1)^{2} \\
& =n(n+2)^{2}, \quad i \neq j, \\
& =3 n\left(w_{i j}^{4}\right)=\sum_{\alpha, \beta, \gamma, \delta}^{n} \mathrm{E}\left(v_{i \alpha} v_{i \beta} v_{i \gamma} v_{i \delta}\right) \mathrm{E}\left(v_{j \alpha} v_{j \beta} v_{j \delta} v_{j \gamma}\right) \\
& =n(n+2), \quad i \neq j \neq k, \\
\mathrm{E}\left(w_{i j}^{2} w_{i k}^{2}\right) & =\sum_{\alpha, \beta}^{n} \mathrm{E}\left(v_{i \alpha}^{2} v_{i \beta}^{2}\right) \mathrm{E}\left(v_{j \alpha}^{2}\right) \mathrm{E}\left(v_{k \beta}^{2}\right) \\
& =3 n+n(n-1) \\
& 3 n(n-1) \\
& 3 n+2) \\
& \\
&
\end{aligned}
$$

1361 where intervening equations can be confirmed by direct enumeration of the nonzero moments.

$$
\mathrm{E}\left[\operatorname{tr}\left(\mathbf{A}^{2}\right)\right]=\mathrm{E}\left[\operatorname{tr}\left(\boldsymbol{\Lambda} \mathbf{V}^{T} \mathbf{V} \boldsymbol{\Lambda} \mathbf{V}^{T} \mathbf{V}\right)\right]
$$




$$
\begin{aligned}
& =\mathrm{E}\left(\sum_{i, j}^{n} \lambda_{i} \lambda_{j} w_{i j}^{2}\right) \\
& =\sum_{i}^{n} \lambda_{i}^{2} \mathrm{E}\left(w_{i i}^{2}\right)+\sum_{i \neq j}^{n} \lambda_{i} \lambda_{j} \mathrm{E}\left(w_{i j}^{2}\right) \\
& =n(n+2) \sum_{i}^{n} \lambda_{i}^{2}+n \sum_{i \neq j}^{n} \lambda_{i} \lambda_{j},
\end{aligned}
$$

$$
\mathrm{E}\left[(\operatorname{tr} \mathbf{A})^{2}\right]=\mathrm{E}\left[\left[\operatorname{tr}\left(\boldsymbol{\Lambda} \mathbf{V}^{T} \mathbf{V}\right)\right]^{2}\right]
$$

$$
\begin{aligned}
& =\mathrm{E}\left(\sum_{i, j}^{n} \lambda_{i} \lambda_{j} w_{i i} w_{j j}\right) \\
& =\sum_{i}^{n} \lambda_{i}^{2} \mathrm{E}\left(w_{i i}^{2}\right)+\sum_{i \neq j}^{n} \lambda_{i} \lambda_{j} \mathrm{E}\left(w_{i i}\right) \mathrm{E}\left(w_{j j}\right) \\
& =n(n+2) \sum_{i}^{n} \lambda_{i}^{2}+n^{2} \sum_{i \neq j}^{n} \lambda_{i} \lambda_{j}
\end{aligned}
$$

\section{$\mathrm{E}\left[\left[\operatorname{tr}\left(\mathbf{A}^{2}\right)\right]^{2}\right]=\mathrm{E}\left[\left[\operatorname{tr}\left(\boldsymbol{\Lambda} \mathbf{V}^{T} \mathbf{V} \boldsymbol{\Lambda} \mathbf{V}^{T} \mathbf{V}\right)\right]^{2}\right]$}

$$
\begin{aligned}
& =\mathrm{E}\left(\sum_{i, j, k, l}^{n} \lambda_{i} \lambda_{j} \lambda_{k} \lambda_{l} w_{i j}^{2} w_{k l}^{2}\right) \\
& =\sum_{i}^{n} \lambda_{i}^{4} \mathrm{E}\left(w_{i i}^{4}\right)+4 \sum_{i \neq j}^{n} \lambda_{i}^{3} \lambda_{j} \mathrm{E}\left(w_{i i}^{2} w_{i j}^{2}\right) \\
& +\sum_{i \neq j}^{n} \lambda_{i}^{2} \lambda_{j}^{2}\left[\mathrm{E}\left(w_{i i}^{2}\right) \mathrm{E}\left(w_{j j}^{2}\right)+2 \mathrm{E}\left(w_{i j}^{4}\right)\right] \\
& +\sum_{i \neq j \neq k}^{n} \lambda_{i}^{2} \lambda_{j} \lambda_{k}\left[2 \mathrm{E}\left(w_{i i}^{2}\right) \mathrm{E}\left(w_{j k}^{2}\right)+4 \mathrm{E}\left(w_{i j}^{2} w_{i k}^{2}\right)\right] \\
& +\sum_{i \neq j \neq k \neq l}^{n} \lambda_{i} \lambda_{j} \lambda_{k} \lambda_{l} \mathrm{E}\left(w_{i j}^{2}\right) \mathrm{E}\left(w_{k l}^{2}\right)
\end{aligned}
$$




$$
\begin{aligned}
& =n(n+2)(n+4)(n+6) \sum_{i}^{n} \lambda_{i}^{4}+4 n(n+2)(n+4) \sum_{i \neq j}^{n} \lambda_{i}^{3} \lambda_{j} \\
& +n(n+2)\left(n^{2}+2 n+6\right) \sum_{i \neq j}^{n} \lambda_{i}^{2} \lambda_{j}^{2}+2 n(n+2) \sum_{i \neq j \neq k}^{n} \lambda_{i}^{2} \lambda_{j} \lambda_{k} \\
& +n^{2} \sum_{i \neq j \neq k \neq l}^{n} \lambda_{i} \lambda_{j} \lambda_{k} \lambda_{l},
\end{aligned}
$$

$$
\begin{aligned}
\mathrm{E}\left[(\operatorname{tr} \mathbf{A})^{4}\right] & =\mathrm{E}\left[\left[\operatorname{tr}\left(\boldsymbol{\Lambda} \mathbf{V}^{T} \mathbf{V}\right)\right]^{4}\right] \\
& =\mathrm{E}\left(\sum_{i, j, k, l}^{n} \lambda_{i} \lambda_{j} \lambda_{k} \lambda_{l} w_{i i} w_{j j} w_{k k} w_{l l}\right) \\
& =\sum_{i}^{n} \lambda_{i}^{4} \mathrm{E}\left(w_{i i}^{4}\right)+4 \sum_{i \neq j}^{n} \lambda_{i}^{3} \lambda_{j} \mathrm{E}\left(w_{i i}^{3}\right) \mathrm{E}\left(w_{j j}\right)+3 \sum_{i \neq j}^{n} \lambda_{i}^{2} \lambda_{j}^{2} \mathrm{E}\left(w_{i i}^{2}\right) \mathrm{E}\left(w_{j j}^{2}\right) \\
& +6 \sum_{i \neq j \neq k}^{n} \lambda_{i}^{2} \lambda_{j} \lambda_{k} \mathrm{E}\left(w_{i i}^{2}\right) \mathrm{E}\left(w_{j j}\right) \mathrm{E}\left(w_{k k}\right) \\
& +\sum_{i \neq j \neq k \neq l}^{n} \lambda_{i} \lambda_{j} \lambda_{k} \lambda_{l} \mathrm{E}\left(w_{i i}\right) \mathrm{E}\left(w_{j j}\right) \mathrm{E}\left(w_{k k}\right) \mathrm{E}\left(w_{l l}\right) \\
& =n(n+2)(n+4)(n+6) \sum_{i}^{n} \lambda_{i}^{4}+4 n^{2}(n+2)(n+4) \sum_{i \neq j}^{n} \lambda_{i}^{3} \lambda_{j} \\
& +3 n^{2}(n+2)^{2} \sum_{i \neq j}^{n} \lambda_{i}^{2} \lambda_{j}^{2}+6 n^{3}(n+2) \sum_{i \neq j \neq k}^{n} \lambda_{i}^{2} \lambda_{j} \lambda_{k} \\
& n_{i} \lambda_{j} \lambda_{k} \lambda_{l}, \\
& \\
&
\end{aligned}
$$$$
\mathrm{E}\left[\operatorname{tr}\left(\mathbf{A}^{2}\right) \cdot(\operatorname{tr} \mathbf{A})^{2}\right]=\mathrm{E}\left[\operatorname{tr}\left(\boldsymbol{\Lambda} \mathbf{V}^{T} \mathbf{V} \boldsymbol{\Lambda} \mathbf{V}^{T} \mathbf{V}\right) \cdot\left[\operatorname{tr}\left(\boldsymbol{\Lambda} \mathbf{V}^{T} \mathbf{V}\right)\right]^{2}\right]
$$$$
=\mathrm{E}\left(\sum_{i, j, k, l}^{n} \lambda_{i} \lambda_{j} \lambda_{k} \lambda_{l} w_{i j}^{2} w_{k k} w_{l l}\right)
$$ 


$$
\begin{aligned}
& =\sum_{i}^{n} \lambda_{i}^{4} \mathrm{E}\left(w_{i i}^{4}\right)+\sum_{i \neq j}^{n} \lambda_{i}^{3} \lambda_{j}\left[2 \mathrm{E}\left(w_{i i}^{3}\right) \mathrm{E}\left(w_{j j}\right)+2 \mathrm{E}\left(w_{i i}^{2} w_{i j}^{2}\right)\right] \\
& +\sum_{i \neq j}^{n} \lambda_{i}^{2} \lambda_{j}^{2}\left[\mathrm{E}\left(w_{i i}^{2}\right) \mathrm{E}\left(w_{j j}^{2}\right)+2 \mathrm{E}\left(w_{i i} w_{j j} w_{i j}^{2}\right)\right] \\
& +\sum_{i \neq j \neq k}^{n} \lambda_{i}^{2} \lambda_{j} \lambda_{k}\left[\mathrm{E}\left(w_{i i}^{2}\right) \mathrm{E}\left(w_{j j}\right) \mathrm{E}\left(w_{k k}\right)+4 \mathrm{E}\left(w_{i i} w_{i j}^{2}\right) \mathrm{E}\left(w_{k k}\right)\right. \\
& \left.+\mathrm{E}\left(w_{i i}^{2}\right) \mathrm{E}\left(w_{j k}^{2}\right)\right]+\sum_{i \neq j \neq k \neq l}^{n} \lambda_{i} \lambda_{j} \lambda_{k} \lambda_{l} \mathrm{E}\left(w_{i j}^{2}\right) \mathrm{E}\left(w_{k k}\right) \mathrm{E}\left(w_{l l}\right) \\
& =n(n+2)(n+4)(n+6) \sum_{i}^{n} \lambda_{i}^{4}+2 n(n+1)(n+2)(n+4) \sum_{i \neq j}^{n} \lambda_{i}^{3} \lambda_{j} \\
& +n(n+2)^{3} \sum_{i \neq j}^{n} \lambda_{i}^{2} \lambda_{j}^{2}+n^{2}(n+2)(n+5) \sum_{i \neq j \neq k}^{n} \lambda_{i}^{2} \lambda_{j} \lambda_{k} \\
& +n^{3} \sum_{i \neq j \neq k \neq l}^{n} \lambda_{i} \lambda_{j} \lambda_{k} \lambda_{l},
\end{aligned}
$$

1364 where notations of the form $i \neq j \neq k \neq l$ represent inequality of every pairwise combination

1365 of the subscripts concerned.

1366 Although equations B6 can be evaluated for any $\boldsymbol{\Sigma}$, calculating the product of all

1367 possible combinations of eigenvalues is rather cumbersome when $p$ is large. For this reason,

1368 it would be preferable to simplify these expressions by noting

$$
\begin{gathered}
\sum_{i}^{n} \lambda_{i}^{r}=\operatorname{tr}\left(\boldsymbol{\Lambda}^{r}\right), \quad r=1,2, \ldots \\
\sum_{i \neq j}^{n} \lambda_{i} \lambda_{j}=\left(\sum_{i=1}^{n} \lambda_{i}\right)^{2}-\sum_{i=1}^{n} \lambda_{i}^{2}=(\operatorname{tr} \boldsymbol{\Lambda})^{2}-\operatorname{tr}\left(\boldsymbol{\Lambda}^{2}\right),
\end{gathered}
$$




$$
\begin{aligned}
\sum_{i \neq j}^{n} \lambda_{i}^{3} \lambda_{j} & =\operatorname{tr} \boldsymbol{\Lambda} \operatorname{tr}\left(\boldsymbol{\Lambda}^{3}\right)-\operatorname{tr}\left(\boldsymbol{\Lambda}^{4}\right) \\
\sum_{i \neq j}^{n} \lambda_{i}^{2} \lambda_{j}^{2} & =3\left[\operatorname{tr}\left(\boldsymbol{\Lambda}^{2}\right)\right]^{2}-\operatorname{tr}\left(\boldsymbol{\Lambda}^{4}\right) \\
\sum_{i \neq j \neq k}^{n} \lambda_{i}^{2} \lambda_{j} \lambda_{k} & =(\operatorname{tr} \boldsymbol{\Lambda})^{2} \operatorname{tr}\left(\boldsymbol{\Lambda}^{2}\right)-2 \operatorname{tr} \boldsymbol{\Lambda} \operatorname{tr}\left(\boldsymbol{\Lambda}^{3}\right)-\left[\operatorname{tr}\left(\boldsymbol{\Lambda}^{2}\right)\right]^{2}+2 \operatorname{tr}\left(\boldsymbol{\Lambda}^{4}\right),
\end{aligned}
$$

1370 Then, equations B6 can be written as follows:

$$
\begin{aligned}
\mathrm{E}\left[\operatorname{tr}\left(\mathbf{A}^{2}\right)\right] & =n(\operatorname{tr} \boldsymbol{\Lambda})^{2}+n(n+1) \operatorname{tr}\left(\boldsymbol{\Lambda}^{2}\right), \\
\mathrm{E}\left[(\operatorname{tr} \mathbf{A})^{2}\right] & =n^{2}(\operatorname{tr} \mathbf{\Lambda})^{2}+2 n \operatorname{tr}\left(\boldsymbol{\Lambda}^{2}\right), \\
\mathrm{E}\left[\left[\operatorname{tr}\left(\mathbf{A}^{2}\right)\right]^{2}\right] & =n^{2}(\operatorname{tr} \boldsymbol{\Lambda})^{4}+2 n\left(n^{2}+n+4\right)(\operatorname{tr} \boldsymbol{\Lambda})^{2} \operatorname{tr}\left(\boldsymbol{\Lambda}^{2}\right)+16 n(n+1) \operatorname{tr} \boldsymbol{\Lambda} \operatorname{tr}\left(\boldsymbol{\Lambda}^{3}\right) \\
& +n\left(n^{3}+2 n^{2}+5 n+4\right)\left[\operatorname{tr}\left(\boldsymbol{\Lambda}^{2}\right)\right]^{2}+4 n\left(2 n^{2}+5 n+5\right) \operatorname{tr}\left(\boldsymbol{\Lambda}^{4}\right), \\
\mathrm{E}\left[(\operatorname{tr} \mathbf{A})^{4}\right] & =n^{4}(\operatorname{tr} \boldsymbol{\Lambda})^{4}+12 n^{3}(\operatorname{tr} \boldsymbol{\Lambda})^{2} \operatorname{tr}\left(\boldsymbol{\Lambda}^{2}\right)+12 n^{2} \operatorname{tr} \boldsymbol{\Lambda} \operatorname{tr}\left(\boldsymbol{\Lambda}^{3}\right) \\
& +32 n^{2}\left[\operatorname{tr}\left(\boldsymbol{\Lambda}^{2}\right)\right]^{2}+48 n \operatorname{tr}\left(\boldsymbol{\Lambda}^{4}\right), \\
\mathrm{E}\left[\operatorname{tr}\left(\mathbf{A}^{2}\right) \cdot(\operatorname{tr} \mathbf{A})^{2}\right] & =n^{3}(\operatorname{tr} \mathbf{\Lambda})^{4}+n^{2}\left(n^{2}+n+10\right)(\operatorname{tr} \boldsymbol{\Lambda})^{2} \operatorname{tr}\left(\boldsymbol{\Lambda}^{2}\right) \\
& +8 n\left(n^{2}+n+2\right) \operatorname{tr} \boldsymbol{\Lambda} \operatorname{tr}\left(\boldsymbol{\Lambda}^{3}\right)+2 n\left(n^{2}+n+4\right)\left[\operatorname{tr}\left(\boldsymbol{\Lambda}^{2}\right)\right]^{2} \\
& +24 n(n+1) \operatorname{tr}\left(\boldsymbol{\Lambda}^{4}\right) .
\end{aligned}
$$

1372 Finally,

$$
\begin{aligned}
\operatorname{Var}\left[\operatorname{tr}\left(\mathbf{A}^{2}\right)\right] & =\mathrm{E}\left[\left[\operatorname{tr}\left(\mathbf{A}^{2}\right)\right]^{2}\right]-\mathrm{E}\left[\operatorname{tr}\left(\mathbf{A}^{2}\right)\right]^{2} \\
& =8 n(\operatorname{tr} \boldsymbol{\Lambda})^{2} \operatorname{tr}\left(\mathbf{\Lambda}^{2}\right)+16 n(n+1) \operatorname{tr} \boldsymbol{\Lambda} \operatorname{tr}\left(\mathbf{\Lambda}^{3}\right) \\
& +4 n(n+1)\left[\operatorname{tr}\left(\mathbf{\Lambda}^{2}\right)\right]^{2}+4 n\left(2 n^{2}+5 n+5\right) \operatorname{tr}\left(\mathbf{\Lambda}^{4}\right) \\
& =8 n^{3}(\operatorname{tr} \boldsymbol{\Lambda})^{2} \operatorname{tr}\left(\boldsymbol{\Lambda}^{2}\right)+32 n^{2} \operatorname{tr} \boldsymbol{\Lambda} \operatorname{tr}\left(\mathbf{\Lambda}^{3}\right)+8 n^{2}\left[\operatorname{tr}\left(\mathbf{\Lambda}^{2}\right)\right]^{2} \\
& +48 n \operatorname{tr}\left(\mathbf{\Lambda}^{4}\right)
\end{aligned}
$$




$$
\begin{aligned}
& =8 n^{2}(\operatorname{tr} \boldsymbol{\Lambda})^{2} \operatorname{tr}\left(\boldsymbol{\Lambda}^{2}\right)+8 n\left(n^{2}+n+2\right) \operatorname{tr} \boldsymbol{\Lambda} \operatorname{tr}\left(\boldsymbol{\Lambda}^{3}\right)+8 n\left[\operatorname{tr}\left(\boldsymbol{\Lambda}^{2}\right)\right]^{2} \\
& +24 n(n+1) \operatorname{tr}\left(\boldsymbol{\Lambda}^{4}\right)
\end{aligned}
$$

1374 Inserting equations B8 and B9 into equations 12, 19, and 32 yields the desired results.

1375 Identical results can be derived from del Waal \& Nel's (1973) results on the

1376 expectations of elementary symmetric functions of eigenvalues and their products for a

1377 Wishart matrix. However, these results appear to have been proved only under the condition

$1378 n>p-1$ (see also Constantine, 1963; Muirhead, 1982: chapter 7). The above derivation is

1379 valid for any combination of $p$ and $n$.

1380

\section{Appendix C}

1382 This part demonstrates that $\operatorname{Cov}\left(r_{i j}^{2}, r_{k l}^{2}\right)=0$ for $(i, j) \neq(k, l)$ under the condition $\mathbf{P}=\mathbf{I}_{p}$, as 1383 cursorily mentioned by Schott (2005). Under this condition, a sample covariance can be

1384 written as $s_{i j}=n_{*}^{-1}\left(\sigma_{i i} \sigma_{j j}\right)^{1 / 2} \mathbf{v}_{i}^{T} \mathbf{v}_{j}$, with $\mathbf{v}_{i}$ and $\mathbf{v}_{j}$ being i.i.d. $N_{n}\left(\mathbf{0}, \mathbf{I}_{n}\right)$. Therefore, a sample

1385 correlation coefficient can be written as $r_{i j}=s_{i j}\left(s_{i i} s_{j j}\right)^{-1 / 2}=\mathbf{u}_{i}^{T} \mathbf{u}_{j}$, where $\mathbf{u}_{i}=$

$1386\left(\mathbf{v}_{i}^{T} \mathbf{v}_{i}\right)^{-1 / 2} \mathbf{v}_{i}$ are uniformly distributed on the surface of the unit hypersphere in the $n$ -

1387 dimensional space. By noting $\mathbf{u}_{i}^{T} \mathbf{u}_{i}=1$, it is possible to see $\mathrm{E}\left(\mathbf{u}_{i} \mathbf{u}_{i}^{T}\right)=n^{-1} \mathbf{I}_{n}$ for any $i$,

1388 because the elements of $\mathbf{u}_{i}$ are symmetric and uncorrelated with one another (a formal

1389 demonstration probably requires introduction of the density function; see Anderson, 2003: p.

1390 49). With these preliminaries, it is easily seen, for $i \neq j \neq k$,

$$
\begin{aligned}
\mathrm{E}\left(r_{i j}^{2} r_{i k}^{2}\right) & =\mathrm{E}\left(\mathbf{u}_{i}^{T} \mathbf{u}_{j} \mathbf{u}_{j}^{T} \mathbf{u}_{i} \mathbf{u}_{i}^{T} \mathbf{u}_{k} \mathbf{u}_{k}^{T} \mathbf{u}_{i}\right) \\
& =\mathrm{E}\left[\mathbf{u}_{i}^{T} \mathrm{E}\left(\mathbf{u}_{j} \mathbf{u}_{j}^{T}\right) \mathbf{u}_{i} \mathbf{u}_{i}^{T} \mathrm{E}\left(\mathbf{u}_{k} \mathbf{u}_{k}^{T}\right) \mathbf{u}_{i}\right] \\
& =n^{-2} \mathrm{E}\left[\mathbf{u}_{i}^{T} \mathbf{u}_{i} \mathbf{u}_{i}^{T} \mathbf{u}_{i}\right] \\
& =n^{-2}=\mathrm{E}\left(r_{i j}^{2}\right) \mathrm{E}\left(r_{i k}^{2}\right)
\end{aligned}
$$


1392 The second equation is valid because $\mathbf{u}_{i}, \mathbf{u}_{j}$, and $\mathbf{u}_{k}$ are stochastically independent from one

1393 another. Therefore, $\operatorname{Cov}\left(r_{i j}^{2}, r_{i k}^{2}\right)=0$ for partly overlapping subscripts. Similarly,

$1394 \operatorname{Cov}\left(r_{i j}^{2}, r_{k l}^{2}\right)=0$ for non-overlapping subscripts, although this could also be seen as a direct

1395 consequence of the independence between $r_{i j}$ and $r_{k l}$ in this case.

\section{Appendix D}

1398 This part outlines assumptions under the heuristic approximation of $\operatorname{Cov}\left(r_{i j}^{2}, r_{k l}^{2}\right)$ (eq. 37).

1399 Pan \& Frank (2004) derived approximate moments for the product of two correlation

1400 coefficients with overlapping subscripts (i.e., $r_{i j}$ and $r_{i k}$ ). Equivalent results for general

1401 combinations of subscripts can be obtained by following the derivation up to their equation

1402 3.8. The relevant result is:

$$
\mathrm{E}\left(r_{i j}^{2} r_{k l}^{2}\right) \approx \mathrm{E}\left(r_{i j}^{2}\right) \mathrm{E}\left(r_{k l}^{2}\right)+4 \mathrm{E}\left(r_{i j}\right) \mathrm{E}\left(r_{k l}\right) \operatorname{Cov}\left(r_{i j}, r_{k l}\right)+2\left[\operatorname{Cov}\left(r_{i j}, r_{k l}\right)\right]^{2}
$$

1404 Derivation of this result only requires the assumption that the third and fourth moments of

1405 correlation coefficients approximately behaves like normal moments; namely, $\mathrm{E}\left(\left[r_{\alpha}-\right.\right.$

$\left.\left.\mathrm{E}\left(r_{\alpha}\right)\right]\left[r_{\beta}-\mathrm{E}\left(r_{\beta}\right)\right]\left[r_{\gamma}-\mathrm{E}\left(r_{\gamma}\right)\right]\right) \approx 0$ and $\mathrm{E}\left(\left[r_{\alpha}-\mathrm{E}\left(r_{\alpha}\right)\right]\left[r_{\beta}-\mathrm{E}\left(r_{\beta}\right)\right]\left[r_{\gamma}-\mathrm{E}\left(r_{\gamma}\right)\right]\left[r_{\delta}-\right.\right.$

$\left.\left.\mathrm{E}\left(r_{\delta}\right)\right]\right) \approx \operatorname{Cov}\left(r_{\alpha}, r_{\beta}\right) \operatorname{Cov}\left(r_{\gamma}, r_{\delta}\right)+\operatorname{Cov}\left(r_{\alpha}, r_{\gamma}\right) \operatorname{Cov}\left(r_{\beta}, r_{\delta}\right)+\operatorname{Cov}\left(r_{\alpha}, r_{\delta}\right) \operatorname{Cov}\left(r_{\beta}, r_{\gamma}\right)$, where

$1408 \alpha, \beta, \gamma$, and $\delta$ are arbitrary pairs of subscripts. Of course these do not strictly hold, except

1409 asymptotically under $n \rightarrow \infty$ (e.g., Olkin \& Siotani, 1976; Konishi, 1979). Equation 37 is

1410 immediate from equation D1. 


\section{Appendix E}

1413 In this part, an asymptotic expression for the variance of $V_{\text {rel }}(\mathbf{R})$ is derived for arbitrary non-

1414 null conditions with $p>2$. Konishi (1979) gave an asymptotic theory for the distribution of

1415 an arbitrary function of eigenvalues of a sample correlation matrix $f\left(l_{1}, \ldots, l_{p}\right)$ under

1416 multivariate normality. In particular, when $n \rightarrow \infty, \sqrt{n}\left[f\left(l_{1}, \ldots, l_{p}\right)-f\left(\lambda_{1}, \ldots, \lambda_{p}\right)\right]$ was

1417 shown to be normally distributed with mean 0 and variance

$$
\tau^{2}=2 \sum_{\alpha, \beta=1}^{p} \lambda_{\alpha} \lambda_{\beta}\left[\delta_{\alpha \beta}-\left(\lambda_{\alpha}+\lambda_{\beta}\right) \sum_{i=1}^{p} \mathrm{v}_{i \alpha}^{2} \mathrm{v}_{i \beta}^{2}+\sum_{i, j=1}^{p} \rho_{i j}^{2} \mathrm{v}_{i \alpha}^{2} \mathrm{v}_{j \beta}^{2}\right] f_{\alpha} f_{\beta},
$$

1419 where the summations are over all combinations of subscripts, $\delta_{\alpha \beta}$ is the Kronecker delta, $v_{i \alpha}$

1420 is the $(i, \alpha)$-th element of the population eigenvector matrix $\mathbf{Y}$, and

$1421 f_{\alpha}=\partial f /\left.\partial l_{\alpha}\right|_{\left(l_{1}, \ldots, l_{p}\right)=\left(\lambda_{1}, \ldots, \lambda_{p}\right)}$, the partial derivative of $f$ with respect to $l_{\alpha}$ evaluated at

$1422\left(l_{1}, \ldots, l_{p}\right)=\left(\lambda_{1}, \ldots, \lambda_{p}\right)$. Note that Konishi's (1979; corollary 2.2) original notation also

1423 concerned potential multiplicity of population eigenvalues, which is ignored here for

1424 simplicity; the population eigenvectors corresponding to multiplicated eigenvalues can in

1425 practice be chosen arbitrarily as a suite of orthogonal vectors in the appropriate subspace, as

1426 is done in numerical determination of eigenvectors. The derivative of $V_{\text {rel }}(\mathbf{R})$ is simply

$$
f_{\alpha}=\left.\frac{\partial}{\partial l_{\alpha}} V_{\mathrm{rel}}(\mathbf{R})\right|_{\left(l_{1}, \ldots, l_{p}\right)=\left(\lambda_{1}, \ldots, \lambda_{p}\right)}=\frac{2}{p(p-1)} \lambda_{\alpha}
$$

1428 Inserting equation E2 into equation E1, we obtain $\tau^{2} / n$ as an asymptotic expression of the

1429 variance of $V_{\text {rel }}(\mathbf{R})$ (eq. 39). It should be noted that this expression will be inaccurate around

$1430 \boldsymbol{P}=\boldsymbol{\Lambda}=\mathbf{I}_{p}$. This is because, under this condition, we can arbitrarily put $\mathbf{Y}=\mathbf{I}_{p}$ to obtain

$1431 \tau^{2}=0$ from equation E1, which is clearly untrue for finite $n$. 
1432 An empirically equivalent result can be obtained from the alternative expression of

$1433 V_{\text {rel }}(\mathbf{R})$ as average squared correlation coefficients (eq. 11), from a similar theory for

1434 functions of a sample correlation matrix by Konishi (1979: theorem 6.2). However, that

1435 alternative expression does not seem to bear much practical advantage, for it typically takes

1436 substantially more computational time to evaluate as $p$ grows.

1437 
1438 Table 1. Summary of selected simulation results for eigenvalue variance of covariance

1439 matrix $V(\mathbf{S})$. Theoretical expectation $(\mathrm{E}[V(\mathbf{S})])$ and standard deviation $(\mathrm{SD}[V(\mathbf{S})])$, as well as

1440 empirical median, mean, standard deviation (ESD), and bias of mean in standard error unit

$1441\left(T=\sqrt{5000}\left\{\right.\right.$ Mean $\left.-\mathrm{E}\left[V_{\text {rel }}(\mathrm{S})\right]\right\} / \mathrm{ESD}$, which should roughly follow $t$ distribution with

14424999 degrees of freedom if the expectation is exact) from 5000 simulation runs are shown for

1443 selected conditions. See Table S1 for full results.

\begin{tabular}{|c|c|c|c|c|c|c|}
\hline & $\bar{E}[V(\mathbf{S})]$ & $\mathrm{SD}[V(\mathbf{S})]$ & Median & Mean & ESD & $T$ \\
\hline \multicolumn{7}{|c|}{$p=2, V(\boldsymbol{\Sigma})=0$} \\
\hline$N=8$ & 0.2857 & 0.3582 & 0.1731 & 0.2876 & 0.3464 & 0.3845 \\
\hline$N=16$ & 0.1333 & 0.1501 & 0.0876 & 0.1330 & 0.1466 & -0.1703 \\
\hline$N=32$ & 0.0645 & 0.0686 & 0.0438 & 0.0646 & 0.0689 & 0.0489 \\
\hline$N=64$ & 0.0317 & 0.0327 & 0.0221 & 0.0315 & 0.0315 & -0.6336 \\
\hline \multicolumn{7}{|c|}{$p=4, V(\boldsymbol{\Sigma})=0$} \\
\hline$N=8$ & 0.2143 & 0.1551 & 0.1734 & 0.2131 & 0.1534 & -0.5437 \\
\hline$N=16$ & 0.1000 & 0.0602 & 0.0861 & 0.0996 & 0.0603 & -0.4826 \\
\hline$N=32$ & 0.0484 & 0.0261 & 0.0429 & 0.0481 & 0.0261 & -0.8676 \\
\hline$N=64$ & 0.0238 & 0.0120 & 0.0216 & 0.0239 & 0.0119 & 0.5321 \\
\hline \multicolumn{7}{|c|}{$p=16, V(\boldsymbol{\Sigma})=0$} \\
\hline$N=8$ & 0.1518 & 0.0304 & 0.1482 & 0.1510 & 0.0307 & -1.8948 \\
\hline$N=16$ & 0.0708 & 0.0102 & 0.0702 & 0.0708 & 0.0102 & -0.0003 \\
\hline$N=32$ & 0.0343 & 0.0038 & 0.0341 & 0.0342 & 0.0037 & -0.4806 \\
\hline$N=64$ & 0.0169 & 0.0015 & 0.0168 & 0.0169 & 0.0015 & -0.6023 \\
\hline \multicolumn{7}{|c|}{$p=64, V(\boldsymbol{\Sigma})=0$} \\
\hline$N=8$ & 0.1473 & 0.0203 & 0.1464 & 0.1477 & 0.0204 & 1.2947 \\
\hline$N=16$ & 0.0688 & 0.0067 & 0.0688 & 0.0689 & 0.0066 & 1.7306 \\
\hline$N=32$ & 0.0333 & 0.0024 & 0.0332 & 0.0333 & 0.0024 & 0.6721 \\
\hline
\end{tabular}


bioRxiv preprint doi: https://doi.org/10.1101/2021.06.19.449119; this version posted August 7, 2021. The copyright holder for this preprint (which was not certified by peer review) is the author/funder, who has granted bioRxiv a license to display the preprint in perpetuity. It is made available under aCC-BY-NC-ND 4.0 International license.

Table 1 (continued)

\begin{tabular}{|c|c|c|c|c|c|c|}
\hline & $\overline{\mathrm{E}[V(\mathbf{S})]}$ & $\mathrm{SD}[V(\mathbf{S})]$ & Median & Mean & ESD & $T$ \\
\hline$N=64$ & 0.0164 & 0.0009 & 0.0163 & 0.0164 & 0.0009 & -0.4407 \\
\hline \multicolumn{7}{|c|}{$p=256, V(\boldsymbol{\Sigma})=0$} \\
\hline$N=8$ & 0.1440 & 0.0097 & 0.1436 & 0.1440 & 0.0099 & 0.1215 \\
\hline$N=16$ & 0.0672 & 0.0031 & 0.0672 & 0.0672 & 0.0031 & 0.9739 \\
\hline$N=32$ & 0.0325 & 0.0011 & 0.0325 & 0.0325 & 0.0011 & -0.8132 \\
\hline$N=64$ & 0.0160 & 0.0004 & 0.0160 & 0.0160 & 0.0004 & 0.0842 \\
\hline \multicolumn{7}{|c|}{$p=1024, V(\Sigma)=0$} \\
\hline$N=8$ & 0.1431 & 0.0048 & 0.1430 & 0.1431 & 0.0048 & -0.6874 \\
\hline$N=16$ & 0.0668 & 0.0015 & 0.0668 & 0.0668 & 0.0015 & 1.7324 \\
\hline$N=32$ & 0.0323 & 0.0005 & 0.0323 & 0.0323 & 0.0005 & 0.3227 \\
\hline$N=64$ & 0.0159 & 0.0002 & 0.0159 & 0.0159 & 0.0002 & 0.3522 \\
\hline \multicolumn{7}{|c|}{$p=2, q=1, V(\boldsymbol{\Sigma})=0.4$} \\
\hline$N=8$ & 0.6857 & 0.8717 & 0.3967 & 0.6901 & 0.8663 & 0.3572 \\
\hline$N=16$ & 0.5333 & 0.4852 & 0.3972 & 0.5426 & 0.4981 & 1.3183 \\
\hline$N=32$ & 0.4645 & 0.3023 & 0.3936 & 0.4616 & 0.3011 & -0.6854 \\
\hline$N=64$ & 0.4317 & 0.2002 & 0.4044 & 0.4337 & 0.2019 & 0.6886 \\
\hline \multicolumn{7}{|c|}{$p=4, q=1, V(\boldsymbol{\Sigma})=0.4$} \\
\hline$N=8$ & 0.6429 & 0.7343 & 0.3949 & 0.6372 & 0.7364 & -0.5426 \\
\hline$N=16$ & 0.5133 & 0.4137 & 0.3968 & 0.5132 & 0.4183 & -0.0225 \\
\hline$N=32$ & 0.4548 & 0.2598 & 0.3937 & 0.4544 & 0.2661 & -0.1087 \\
\hline$N=64$ & 0.4270 & 0.1728 & 0.4025 & 0.4292 & 0.1756 & 0.8791 \\
\hline \multicolumn{7}{|c|}{$p=16, q=1, V(\boldsymbol{\Sigma})=0.4$} \\
\hline$N=8$ & 0.6107 & 0.6427 & 0.4130 & 0.6165 & 0.6509 & 0.6282 \\
\hline$N=16$ & 0.4983 & 0.3668 & 0.4060 & 0.5036 & 0.3748 & 0.9922 \\
\hline$N=32$ & 0.4476 & 0.2322 & 0.4057 & 0.4492 & 0.2314 & 0.5001 \\
\hline$N=64$ & 0.4234 & 0.1552 & 0.4005 & 0.4227 & 0.1546 & -0.3205 \\
\hline
\end{tabular}


Table 1 (continued)

\begin{tabular}{|c|c|c|c|c|c|c|}
\hline & $\mathrm{E}[V(\mathbf{S})]$ & $\mathrm{SD}[V(\mathbf{s})]$ & Median & Mean & ESD & $T$ \\
\hline \multicolumn{7}{|c|}{$p=64, q=1, V(\boldsymbol{\Sigma})=0.4$} \\
\hline$N=8$ & 0.6027 & 0.6206 & 0.4227 & 0.6052 & 0.5988 & 0.2997 \\
\hline$N=16$ & 0.4946 & 0.3554 & 0.4061 & 0.5021 & 0.3631 & 1.4613 \\
\hline$N=32$ & 0.4458 & 0.2255 & 0.4003 & 0.4445 & 0.2244 & -0.4124 \\
\hline$N=64$ & 0.4225 & 0.1509 & 0.3986 & 0.4237 & 0.1546 & 0.5322 \\
\hline \multicolumn{7}{|c|}{$p=256, q=1, V(\boldsymbol{\Sigma})=0.4$} \\
\hline$N=8$ & 0.6007 & 0.6151 & 0.4140 & 0.6076 & 0.6137 & 0.7995 \\
\hline$N=16$ & 0.4936 & 0.3526 & 0.4000 & 0.4873 & 0.3492 & -1.2751 \\
\hline$N=32$ & 0.4453 & 0.2239 & 0.4068 & 0.4465 & 0.2213 & 0.3813 \\
\hline$N=64$ & 0.4223 & 0.1499 & 0.4003 & 0.4234 & 0.1505 & 0.5054 \\
\hline \multicolumn{7}{|c|}{$p=1024, q=1, V(\boldsymbol{\Sigma})=0.4$} \\
\hline$N=8$ & 0.6002 & 0.6138 & 0.3961 & 0.5940 & 0.6361 & -0.6814 \\
\hline$N=16$ & 0.4934 & 0.3519 & 0.4015 & 0.4977 & 0.3638 & 0.8432 \\
\hline$N=32$ & 0.4452 & 0.2235 & 0.4037 & 0.4489 & 0.2304 & 1.1498 \\
\hline$N=64$ & 0.4222 & 0.1496 & 0.4020 & 0.4240 & 0.1526 & 0.8140 \\
\hline \multicolumn{7}{|c|}{$p=2, q=1, V(\boldsymbol{\Sigma})=0.8$} \\
\hline$N=8$ & 1.0857 & 1.2651 & 0.6587 & 1.0792 & 1.3030 & -0.3520 \\
\hline$N=16$ & 0.9333 & 0.7343 & 0.7440 & 0.9364 & 0.7250 & 0.3003 \\
\hline$N=32$ & 0.8645 & 0.4698 & 0.7732 & 0.8675 & 0.4675 & 0.4571 \\
\hline$N=64$ & 0.8317 & 0.3157 & 0.7840 & 0.8333 & 0.3172 & 0.3574 \\
\hline \multicolumn{7}{|c|}{$p=4, q=1, V(\boldsymbol{\Sigma})=0.8$} \\
\hline$N=8$ & 1.0714 & 1.2182 & 0.6903 & 1.0730 & 1.2081 & 0.0917 \\
\hline$N=16$ & 0.9267 & 0.7096 & 0.7454 & 0.9328 & 0.7139 & 0.6117 \\
\hline$N=32$ & 0.8613 & 0.4549 & 0.7682 & 0.8585 & 0.4565 & -0.4368 \\
\hline$N=64$ & 0.8302 & 0.3061 & 0.7903 & 0.8350 & 0.3069 & 1.1137 \\
\hline
\end{tabular}


bioRxiv preprint doi: https://doi.org/10.1101/2021.06.19.449119; this version posted August 7, 2021. The copyright holder for this preprint (which was not certified by peer review) is the author/funder, who has granted bioRxiv a license to display the preprint in perpetuity. It is made available under aCC-BY-NC-ND 4.0 International license.

Table 1 (continued)

\begin{tabular}{|c|c|c|c|c|c|c|}
\hline & $\bar{E}[V(\mathbf{S})]$ & $\mathrm{SD}[V(\mathbf{S})]$ & Median & Mean & ESD & $T$ \\
\hline$N=8$ & 1.0607 & 1.1840 & 0.6778 & 1.0687 & 1.1804 & 0.4765 \\
\hline$N=16$ & 0.9217 & 0.6917 & 0.7517 & 0.9147 & 0.6842 & -0.7221 \\
\hline$N=32$ & 0.8589 & 0.4442 & 0.7847 & 0.8683 & 0.4387 & 1.5248 \\
\hline$N=64$ & 0.8290 & 0.2992 & 0.7925 & 0.8358 & 0.3067 & 1.5855 \\
\hline \multicolumn{7}{|c|}{$p=64, q=1, V(\boldsymbol{\Sigma})=0.8$} \\
\hline$N=8$ & 1.0580 & 1.1756 & 0.6979 & 1.0755 & 1.2081 & 1.0231 \\
\hline$N=16$ & 0.9204 & 0.6873 & 0.7463 & 0.9304 & 0.7024 & 1.0083 \\
\hline$N=32$ & 0.8583 & 0.4415 & 0.7643 & 0.8495 & 0.4352 & -1.4315 \\
\hline$N=64$ & 0.8287 & 0.2975 & 0.7873 & 0.8292 & 0.2977 & 0.1296 \\
\hline \multicolumn{7}{|c|}{$p=256, q=1, V(\boldsymbol{\Sigma})=0.8$} \\
\hline$N=8$ & 1.0574 & 1.1735 & 0.6982 & 1.0818 & 1.1935 & 1.4492 \\
\hline$N=16$ & 0.9201 & 0.6862 & 0.7373 & 0.9122 & 0.6746 & -0.8321 \\
\hline$N=32$ & 0.8581 & 0.4409 & 0.7749 & 0.8663 & 0.4540 & 1.2689 \\
\hline$N=64$ & 0.8286 & 0.2971 & 0.7824 & 0.8219 & 0.2873 & -1.6582 \\
\hline \multicolumn{7}{|c|}{$p=1024, q=1, V(\boldsymbol{\Sigma})=0.8$} \\
\hline$N=8$ & 1.0572 & 1.1729 & 0.6881 & 1.0573 & 1.1723 & 0.0072 \\
\hline$N=16$ & 0.9200 & 0.6859 & 0.7655 & 0.9344 & 0.6943 & 1.4624 \\
\hline$N=32$ & 0.8581 & 0.4407 & 0.7709 & 0.8531 & 0.4414 & -0.7945 \\
\hline$N=64$ & 0.8286 & 0.2969 & 0.7840 & 0.8292 & 0.2987 & 0.1364 \\
\hline
\end{tabular}

1444 
1445 Table 2. Summary of selected simulation results for relative eigenvalue variance of

1446 covariance matrix $V_{\text {rel }}(\mathbf{S})$. (Approximate) theoretical expectation $\left(\mathrm{E}\left[V_{\mathrm{rel}}(\mathbf{S})\right]\right)$ and standard

1447 deviation $\left(\mathrm{SD}\left[V_{\text {rel }}(\mathbf{S})\right]\right)$, as well as empirical median, mean, standard deviation (ESD), and

1448 bias of mean in standard error unit $(T)$ from 5000 simulation runs are shown for selected

1449 conditions. See Table 1 for further information and Table S2 for full results.

\begin{tabular}{|c|c|c|c|c|c|c|}
\hline & $\approx \mathrm{E}\left[V_{\mathrm{rel}}(\mathbf{s})\right]$ & $\approx \mathrm{SD}\left[V_{\mathrm{rel}}(\mathbf{s})\right]$ & Median & Mean & ESD & $T$ \\
\hline \multicolumn{7}{|c|}{$p=2, V_{\text {rel }}(\boldsymbol{\Sigma})=0$} \\
\hline$N=8$ & 0.2500 & 0.1936 & 0.2079 & 0.2514 & 0.1924 & 0.4961 \\
\hline$N=16$ & 0.1250 & 0.1102 & 0.0953 & 0.1259 & 0.1098 & 0.6000 \\
\hline$N=32$ & 0.0625 & 0.0587 & 0.0448 & 0.0628 & 0.0597 & 0.3234 \\
\hline$N=64$ & 0.0313 & 0.0303 & 0.0224 & 0.0309 & 0.0289 & -0.8301 \\
\hline \multicolumn{7}{|c|}{$p=4, V_{\text {rel }}(\boldsymbol{\Sigma})=0$} \\
\hline$N=8$ & 0.2000 & 0.0840 & 0.1856 & 0.2002 & 0.0858 & 0.1644 \\
\hline$N=16$ & 0.0968 & 0.0433 & 0.0907 & 0.0968 & 0.0434 & 0.0620 \\
\hline$N=32$ & 0.0476 & 0.0219 & 0.0438 & 0.0474 & 0.0221 & -0.6146 \\
\hline$N=64$ & 0.0236 & 0.0110 & 0.0218 & 0.0237 & 0.0110 & 0.6544 \\
\hline \multicolumn{7}{|c|}{$p=16, V_{\text {rel }}(\Sigma)=0$} \\
\hline$N=8$ & 0.1579 & 0.0193 & 0.1562 & 0.1580 & 0.0197 & 0.3764 \\
\hline$N=16$ & 0.0744 & 0.0091 & 0.0738 & 0.0746 & 0.0094 & 1.4158 \\
\hline$N=32$ & 0.0361 & 0.0044 & 0.0357 & 0.0361 & 0.0044 & -1.2604 \\
\hline$N=64$ & 0.0178 & 0.0022 & 0.0177 & 0.0178 & 0.0022 & 0.4338 \\
\hline \multicolumn{7}{|c|}{$p=64, V_{\text {rel }}(\Sigma)=0$} \\
\hline$N=8$ & 0.1467 & 0.0047 & 0.1462 & 0.1466 & 0.0046 & -0.6909 \\
\hline$N=16$ & 0.0686 & 0.0022 & 0.0685 & 0.0686 & 0.0022 & 0.5618 \\
\hline$N=32$ & 0.0332 & 0.0010 & 0.0332 & 0.0332 & 0.0011 & 0.0446 \\
\hline$N=64$ & 0.0164 & 0.0005 & 0.0163 & 0.0164 & 0.0005 & -0.5869 \\
\hline
\end{tabular}


Table 2 (continued)

\begin{tabular}{|c|c|c|c|c|c|c|}
\hline & $\approx \mathrm{E}\left[V_{\mathrm{rel}}(\mathbf{S})\right]$ & $\approx \mathrm{SD}\left[V_{\text {rel }}(\mathbf{S})\right]$ & Median & Mean & ESD & $T$ \\
\hline \multicolumn{7}{|c|}{$p=256, V_{\text {rel }}(\Sigma)=0$} \\
\hline$N=8$ & 0.1438 & 0.0012 & 0.1437 & 0.1438 & 0.0012 & -0.0173 \\
\hline$N=16$ & 0.0672 & 0.0005 & 0.0671 & 0.0672 & 0.0005 & 1.4545 \\
\hline$N=32$ & 0.0325 & 0.0003 & 0.0325 & 0.0325 & 0.0003 & -0.7481 \\
\hline$N=64$ & 0.0160 & 0.0001 & 0.0160 & 0.0160 & 0.0001 & 0.3608 \\
\hline \multicolumn{7}{|c|}{$p=1024, V_{\text {rel }}(\Sigma)=0$} \\
\hline$N=8$ & 0.1431 & 0.0003 & 0.1431 & 0.1431 & 0.0003 & -0.5105 \\
\hline$N=16$ & 0.0668 & 0.0001 & 0.0668 & 0.0668 & 0.0001 & 0.0231 \\
\hline$N=32$ & 0.0323 & 0.0001 & 0.0323 & 0.0323 & 0.0001 & -1.3055 \\
\hline$N=64$ & 0.0159 & 0.0000 & 0.0159 & 0.0159 & 0.0000 & -0.0022 \\
\hline \multicolumn{7}{|c|}{$p=2, q=1, V_{\text {rel }}(\Sigma)=0.4$} \\
\hline$N=8$ & 0.4377 & 0.2825 & 0.4939 & 0.4804 & 0.2334 & 12.9356 \\
\hline$N=16$ & 0.4232 & 0.1982 & 0.4457 & 0.4384 & 0.1764 & 6.0670 \\
\hline$N=32$ & 0.4132 & 0.1378 & 0.4169 & 0.4152 & 0.1297 & 1.1224 \\
\hline$N=64$ & 0.4070 & 0.0963 & 0.4108 & 0.4083 & 0.0945 & 0.9214 \\
\hline \multicolumn{7}{|c|}{$p=4, q=1, V_{\text {rel }}(\Sigma)=0.4$} \\
\hline$N=8$ & 0.4319 & 0.2065 & 0.4730 & 0.4675 & 0.1658 & 15.1734 \\
\hline$N=16$ & 0.4200 & 0.1444 & 0.4316 & 0.4288 & 0.1272 & 4.8902 \\
\hline$N=32$ & 0.4113 & 0.1001 & 0.4147 & 0.4125 & 0.0944 & 0.9058 \\
\hline$N=64$ & 0.4060 & 0.0699 & 0.4073 & 0.4067 & 0.0682 & 0.7503 \\
\hline \multicolumn{7}{|c|}{$p=16, q=1, V_{\text {rel }}(\Sigma)=0.4$} \\
\hline$N=8$ & 0.4275 & 0.1691 & 0.4657 & 0.4626 & 0.1346 & 18.4219 \\
\hline$N=16$ & 0.4174 & 0.1175 & 0.4302 & 0.4270 & 0.1049 & 6.4710 \\
\hline$N=32$ & 0.4098 & 0.0813 & 0.4161 & 0.4129 & 0.0762 & 2.8682 \\
\hline$N=64$ & 0.4052 & 0.0566 & 0.4070 & 0.4058 & 0.0548 & 0.7118 \\
\hline
\end{tabular}


bioRxiv preprint doi: https://doi.org/10.1101/2021.06.19.449119; this version posted August 7, 2021. The copyright holder for this preprint (which was not certified by peer review) is the author/funder, who has granted bioRxiv a license to display the preprint in perpetuity. It is made available under aCC-BY-NC-ND 4.0 International license.

Table 2 (continued)

\begin{tabular}{|c|c|c|c|c|c|c|}
\hline & $\approx \mathrm{E}\left[V_{\mathrm{rel}}(\mathbf{S})\right]$ & $\approx \mathrm{SD}\left[V_{\mathrm{rel}}(\mathbf{S})\right]$ & Median & Mean & ESD & $T$ \\
\hline$N=8$ & 0.4264 & 0.1613 & 0.4670 & 0.4599 & 0.1273 & 18.6017 \\
\hline$N=16$ & 0.4168 & 0.1119 & 0.4286 & 0.4266 & 0.0996 & 6.9651 \\
\hline$N=32$ & 0.4095 & 0.0773 & 0.4125 & 0.4109 & 0.0726 & 1.3497 \\
\hline$N=64$ & 0.4050 & 0.0538 & 0.4063 & 0.4056 & 0.0532 & 0.7287 \\
\hline \multicolumn{7}{|c|}{$p=256, q=1, V_{\text {rel }}(\Sigma)=0.4$} \\
\hline$N=8$ & 0.4261 & 0.1594 & 0.4637 & 0.4606 & 0.1249 & 19.5222 \\
\hline$N=16$ & 0.4166 & 0.1105 & 0.4262 & 0.4228 & 0.0977 & 4.4222 \\
\hline$N=32$ & 0.4094 & 0.0763 & 0.4148 & 0.4120 & 0.0718 & 2.5791 \\
\hline$N=64$ & 0.4050 & 0.0531 & 0.4066 & 0.4058 & 0.0520 & 1.1405 \\
\hline \multicolumn{7}{|c|}{$p=1024, q=1, V_{\text {rel }}(\boldsymbol{\Sigma})=0.4$} \\
\hline$N=8$ & 0.4261 & 0.1590 & 0.4566 & 0.4548 & 0.1261 & 16.1041 \\
\hline$N=16$ & 0.4166 & 0.1102 & 0.4272 & 0.4246 & 0.0990 & 5.7314 \\
\hline$N=32$ & 0.4094 & 0.0761 & 0.4140 & 0.4123 & 0.0718 & 2.8690 \\
\hline$N=64$ & 0.4050 & 0.0530 & 0.4068 & 0.4058 & 0.0521 & 1.1494 \\
\hline \multicolumn{7}{|c|}{$p=2, q=1, V_{\text {rel }}(\Sigma)=0.8$} \\
\hline$N=8$ & 0.7847 & 0.1153 & 0.8314 & 0.7947 & 0.1473 & 4.8016 \\
\hline$N=16$ & 0.7929 & 0.0866 & 0.8164 & 0.7963 & 0.1004 & 2.4327 \\
\hline$N=32$ & 0.7969 & 0.0625 & 0.8090 & 0.7990 & 0.0672 & 2.2207 \\
\hline$N=64$ & 0.7986 & 0.0445 & 0.8030 & 0.7983 & 0.0470 & -0.3966 \\
\hline \multicolumn{7}{|c|}{$p=4, q=1, V_{\text {rel }}(\Sigma)=0.8$} \\
\hline$N=8$ & 0.7841 & 0.0920 & 0.8207 & 0.7927 & 0.1170 & 5.1526 \\
\hline$N=16$ & 0.7927 & 0.0692 & 0.8086 & 0.7945 & 0.0793 & 1.6175 \\
\hline$N=32$ & 0.7968 & 0.0498 & 0.8039 & 0.7967 & 0.0537 & -0.1966 \\
\hline$N=64$ & 0.7985 & 0.0354 & 0.8026 & 0.7993 & 0.0368 & 1.5352 \\
\hline \multicolumn{7}{|c|}{$p=16, q=1, V_{\text {rel }}(\Sigma)=0.8$} \\
\hline$N=8$ & 0.7838 & 0.0810 & 0.8153 & 0.7913 & 0.1049 & 5.0688 \\
\hline
\end{tabular}


bioRxiv preprint doi: https://doi.org/10.1101/2021.06.19.449119; this version posted August 7, 2021. The copyright holder for this preprint (which was not certified by peer review) is the author/funder, who has granted bioRxiv a license to display the preprint in perpetuity. It is made available under aCC-BY-NC-ND 4.0 International license.

Table 2 (continued)

\begin{tabular}{|c|c|c|c|c|c|c|}
\hline & $\approx \mathrm{E}\left[V_{\mathrm{rel}}(\mathbf{S})\right]$ & $\approx \mathrm{SD}\left[V_{\text {rel }}(\mathbf{S})\right]$ & Median & Mean & ESD & $T$ \\
\hline$N=16$ & 0.7926 & 0.0609 & 0.8067 & 0.7943 & 0.0696 & 1.7415 \\
\hline$N=32$ & 0.7968 & 0.0437 & 0.8044 & 0.7989 & 0.0452 & 3.3474 \\
\hline$N=64$ & 0.7985 & 0.0310 & 0.8019 & 0.7990 & 0.0323 & 1.0675 \\
\hline \multicolumn{7}{|c|}{$p=64, q=1, V_{\text {rel }}(\Sigma)=0.8$} \\
\hline$N=8$ & 0.7837 & 0.0787 & 0.8157 & 0.7915 & 0.1022 & 5.4258 \\
\hline$N=16$ & 0.7926 & 0.0592 & 0.8072 & 0.7956 & 0.0669 & 3.2349 \\
\hline$N=32$ & 0.7967 & 0.0425 & 0.8021 & 0.7967 & 0.0447 & -0.0106 \\
\hline$N=64$ & 0.7985 & 0.0301 & 0.8018 & 0.7989 & 0.0308 & 0.8231 \\
\hline \multicolumn{7}{|c|}{$p=256, q=1, V_{\text {rel }}(\Sigma)=0.8$} \\
\hline$N=8$ & 0.7836 & 0.0781 & 0.8150 & 0.7930 & 0.0994 & 6.6508 \\
\hline$N=16$ & 0.7926 & 0.0588 & 0.8058 & 0.7944 & 0.0663 & 1.9392 \\
\hline$N=32$ & 0.7967 & 0.0422 & 0.8033 & 0.7976 & 0.0455 & 1.3138 \\
\hline$N=64$ & 0.7985 & 0.0299 & 0.8010 & 0.7983 & 0.0303 & -0.5863 \\
\hline \multicolumn{7}{|c|}{$p=1024, q=1, V_{\text {rel }}(\Sigma)=0.8$} \\
\hline$N=8$ & 0.7836 & 0.0780 & 0.8140 & 0.7904 & 0.1011 & 4.7652 \\
\hline$N=16$ & 0.7926 & 0.0587 & 0.8081 & 0.7959 & 0.0665 & 3.5530 \\
\hline$N=32$ & 0.7967 & 0.0421 & 0.8026 & 0.7967 & 0.0448 & -0.0218 \\
\hline$N=64$ & 0.7985 & 0.0299 & 0.8012 & 0.7987 & 0.0307 & 0.4338 \\
\hline
\end{tabular}

1450 
1451 Table 3. Summary of selected simulation results for relative eigenvalue variance of

1452 correlation matrix $V_{\text {rel }}(\mathbf{R})$. Theoretical expectation $\left(\mathrm{E}\left[V_{\text {rel }}(\mathbf{R})\right]\right)$ and (approximate) standard

1453 deviation ( $\left.\mathrm{SD}\left[V_{\text {rel }}(\mathbf{R})\right]\right)$, as well as empirical median, mean, standard deviation (ESD), and

1454 bias of mean in standard error unit $(T)$ from 5000 simulation runs are shown for selected

1455 conditions. The theoretical standard deviations shown in this table are from heuristic

1456 approximation (eqs. 28, 36-38) except under the null conditions. See Table 1 for further

1457 information and Table S3 for full results.

\begin{tabular}{|c|c|c|c|c|c|c|}
\hline & $\mathrm{E}\left[V_{\text {rel }}(\mathbf{R})\right]$ & $\approx \mathrm{SD}\left[V_{\text {rel }}(\mathbf{R})\right]$ & Median & Mean & ESD & $T$ \\
\hline \multicolumn{7}{|c|}{$p=2, V_{\text {rel }}(\mathbf{P})=0$} \\
\hline$N=8$ & 0.1429 & 0.1650 & 0.0792 & 0.1429 & 0.1635 & 0.0363 \\
\hline$N=16$ & 0.0667 & 0.0856 & 0.0336 & 0.0679 & 0.0862 & 1.0235 \\
\hline$N=32$ & 0.0323 & 0.0435 & 0.0163 & 0.0326 & 0.0427 & 0.6113 \\
\hline$N=64$ & 0.0159 & 0.0219 & 0.0075 & 0.0156 & 0.0205 & -1.0380 \\
\hline \multicolumn{7}{|c|}{$p=4, V_{\text {rel }}(\mathbf{P})=0$} \\
\hline$N=8$ & 0.1429 & 0.0673 & 0.1332 & 0.1432 & 0.0673 & 0.3148 \\
\hline$N=16$ & 0.0667 & 0.0349 & 0.0608 & 0.0661 & 0.0344 & -1.0696 \\
\hline$N=32$ & 0.0323 & 0.0178 & 0.0292 & 0.0321 & 0.0175 & -0.6906 \\
\hline$N=64$ & 0.0159 & 0.0090 & 0.0142 & 0.0157 & 0.0087 & -1.0749 \\
\hline \multicolumn{7}{|c|}{$p=16, V_{\text {rel }}(\mathbf{P})=0$} \\
\hline$N=8$ & 0.1429 & 0.0151 & 0.1411 & 0.1428 & 0.0151 & -0.0917 \\
\hline$N=16$ & 0.0667 & 0.0078 & 0.0663 & 0.0669 & 0.0080 & 1.7743 \\
\hline$N=32$ & 0.0323 & 0.0040 & 0.0319 & 0.0322 & 0.0039 & -1.7517 \\
\hline$N=64$ & 0.0159 & 0.0020 & 0.0158 & 0.0159 & 0.0020 & 0.7127 \\
\hline \multicolumn{7}{|c|}{$p=64, V_{\text {rel }}(\mathbf{P})=0$} \\
\hline$N=8$ & 0.1429 & 0.0037 & 0.1425 & 0.1428 & 0.0036 & -0.4617 \\
\hline$N=16$ & 0.0667 & 0.0019 & 0.0666 & 0.0667 & 0.0019 & 0.4407 \\
\hline
\end{tabular}




\begin{tabular}{|c|c|c|c|c|c|c|}
\hline & $\mathrm{E}\left[V_{\text {rel }}(\mathbf{R})\right]$ & $\approx \mathrm{SD}\left[V_{\mathrm{rel}}(\mathbf{R})\right]$ & Median & Mean & ESD & $T$ \\
\hline$N=32$ & 0.0323 & 0.0010 & 0.0322 & 0.0323 & 0.0010 & -0.0725 \\
\hline$N=64$ & 0.0159 & 0.0005 & 0.0159 & 0.0159 & 0.0005 & -0.6525 \\
\hline \multicolumn{7}{|c|}{$p=256, V_{\text {rel }}(\mathbf{P})=0$} \\
\hline$N=8$ & 0.1429 & 0.0009 & 0.1428 & 0.1429 & 0.0009 & 0.0105 \\
\hline$N=16$ & 0.0667 & 0.0005 & 0.0667 & 0.0667 & 0.0005 & 1.8676 \\
\hline$N=32$ & 0.0323 & 0.0002 & 0.0323 & 0.0323 & 0.0002 & -0.9701 \\
\hline$N=64$ & 0.0159 & 0.0001 & 0.0159 & 0.0159 & 0.0001 & 0.3244 \\
\hline \multicolumn{7}{|c|}{$p=1024, V_{\text {rel }}(\mathbf{P})=0$} \\
\hline$N=8$ & 0.1429 & 0.0002 & 0.1428 & 0.1429 & 0.0002 & -0.9639 \\
\hline$N=16$ & 0.0667 & 0.0001 & 0.0667 & 0.0667 & 0.0001 & 0.5220 \\
\hline$N=32$ & 0.0323 & 0.0001 & 0.0323 & 0.0323 & 0.0001 & -1.8393 \\
\hline$N=64$ & 0.0159 & 0.0000 & 0.0159 & 0.0159 & 0.0000 & -0.0357 \\
\hline \multicolumn{7}{|c|}{$p=2, q=1, V_{\text {rel }}(\mathbf{P})=0.4$} \\
\hline$N=8$ & 0.4318 & 0.2495 & 0.4362 & 0.4294 & 0.2516 & -0.6571 \\
\hline$N=16$ & 0.4111 & 0.1844 & 0.4230 & 0.4147 & 0.1832 & 1.3640 \\
\hline$N=32$ & 0.4046 & 0.1326 & 0.4056 & 0.4041 & 0.1322 & -0.2806 \\
\hline$N=64$ & 0.4021 & 0.0944 & 0.4058 & 0.4025 & 0.0954 & 0.3294 \\
\hline \multicolumn{7}{|c|}{$p=4, q=1, V_{\text {rel }}(\mathbf{P})=0.4$} \\
\hline$N=8$ & 0.4318 & 0.1950 & 0.4314 & 0.4287 & 0.1750 & -1.2271 \\
\hline$N=16$ & 0.4111 & 0.1380 & 0.4141 & 0.4093 & 0.1317 & -0.9769 \\
\hline$N=32$ & 0.4046 & 0.0975 & 0.4054 & 0.4034 & 0.0960 & -0.8743 \\
\hline$N=64$ & 0.4021 & 0.0688 & 0.4033 & 0.4022 & 0.0687 & 0.1270 \\
\hline \multicolumn{7}{|c|}{$p=16, q=1, V_{\text {rel }}(\mathbf{P})=0.4$} \\
\hline$N=8$ & 0.4318 & 0.1621 & 0.4321 & 0.4329 & 0.1391 & 0.5780 \\
\hline$N=16$ & 0.4111 & 0.1130 & 0.4149 & 0.4120 & 0.1073 & 0.5980 \\
\hline$N=32$ & 0.4046 & 0.0793 & 0.4084 & 0.4055 & 0.0773 & 0.7996 \\
\hline
\end{tabular}




\begin{tabular}{|c|c|c|c|c|c|c|}
\hline & $\mathrm{E}\left[V_{\mathrm{rel}}(\mathbf{R})\right]$ & $\approx \mathrm{SD}\left[V_{\mathrm{rel}}(\mathbf{R})\right]$ & Median & Mean & ESD & $\bar{T}$ \\
\hline$N=64$ & 0.4021 & 0.0559 & 0.4030 & 0.4021 & 0.0552 & -0.0283 \\
\hline \multicolumn{7}{|c|}{$p=64, q=1, V_{\text {rel }}(\mathbf{P})=0.4$} \\
\hline$N=8$ & 0.4318 & 0.1542 & 0.4366 & 0.4332 & 0.1304 & 0.7802 \\
\hline$N=16$ & 0.4111 & 0.1074 & 0.4144 & 0.4129 & 0.1015 & 1.2079 \\
\hline$N=32$ & 0.4046 & 0.0754 & 0.4052 & 0.4039 & 0.0733 & -0.6457 \\
\hline$N=64$ & 0.4021 & 0.0531 & 0.4029 & 0.4021 & 0.0535 & 0.0493 \\
\hline \multicolumn{7}{|c|}{$p=256, q=1, V_{\text {rel }}(\mathbf{P})=0.4$} \\
\hline$N=8$ & 0.4318 & 0.1522 & 0.4348 & 0.4343 & 0.1278 & 1.3865 \\
\hline$N=16$ & 0.4111 & 0.1061 & 0.4118 & 0.4091 & 0.0995 & -1.4526 \\
\hline$N=32$ & 0.4046 & 0.0744 & 0.4078 & 0.4052 & 0.0726 & 0.5664 \\
\hline$N=64$ & 0.4021 & 0.0524 & 0.4033 & 0.4024 & 0.0522 & 0.4500 \\
\hline \multicolumn{7}{|c|}{$p=1024, q=1, V_{\text {rel }}(\mathbf{P})=0.4$} \\
\hline$N=8$ & 0.4318 & & 0.4266 & 0.4286 & 0.1287 & -1.7550 \\
\hline$N=16$ & 0.4111 & & 0.4127 & 0.4111 & 0.1008 & -0.0454 \\
\hline$N=32$ & 0.4046 & & 0.4072 & 0.4055 & 0.0726 & 0.8769 \\
\hline$N=64$ & 0.4021 & & 0.4035 & 0.4024 & 0.0524 & 0.4578 \\
\hline \multicolumn{7}{|c|}{$p=2, q=1, V_{\text {rel }}(\mathbf{P})=0.8$} \\
\hline$N=8$ & 0.7831 & 0.1596 & 0.8250 & 0.7853 & 0.1571 & 0.9790 \\
\hline$N=16$ & 0.7918 & 0.1015 & 0.8141 & 0.7928 & 0.1033 & 0.6253 \\
\hline$N=32$ & 0.7961 & 0.0674 & 0.8076 & 0.7976 & 0.0680 & 1.5536 \\
\hline$N=64$ & 0.7981 & 0.0462 & 0.8024 & 0.7976 & 0.0473 & -0.6617 \\
\hline \multicolumn{7}{|c|}{$p=4, q=1, V_{\text {rel }}(\mathbf{P})=0.8$} \\
\hline$N=8$ & 0.7831 & 0.1115 & 0.8149 & 0.7840 & 0.1249 & 0.4605 \\
\hline$N=16$ & 0.7918 & 0.0749 & 0.8059 & 0.7913 & 0.0815 & -0.4682 \\
\hline$N=32$ & 0.7961 & 0.0516 & 0.8027 & 0.7953 & 0.0543 & -1.0173 \\
\hline$N=64$ & 0.7981 & 0.0360 & 0.8019 & 0.7987 & 0.0370 & 1.2077 \\
\hline
\end{tabular}




\begin{tabular}{|c|c|c|c|c|c|c|}
\hline & $\mathrm{E}\left[V_{\mathrm{rel}}(\mathbf{R})\right]$ & $\approx \mathrm{SD}\left[V_{\mathrm{rel}}(\mathbf{R})\right]$ & Median & Mean & ESD & $T$ \\
\hline \multicolumn{7}{|c|}{$p=16, q=1, V_{\text {rel }}(\mathbf{P})=0.8$} \\
\hline$N=8$ & 0.7831 & 0.0927 & 0.8102 & 0.7835 & 0.1116 & 0.2370 \\
\hline$N=16$ & 0.7918 & 0.0638 & 0.8045 & 0.7913 & 0.0716 & -0.5326 \\
\hline$N=32$ & 0.7961 & 0.0445 & 0.8035 & 0.7976 & 0.0457 & 2.4043 \\
\hline$N=64$ & 0.7981 & 0.0313 & 0.8013 & 0.7984 & 0.0325 & 0.7108 \\
\hline \multicolumn{7}{|c|}{$p=64, q=1, V_{\text {rel }}(\mathbf{P})=0.8$} \\
\hline$N=8$ & 0.7831 & 0.0896 & 0.8098 & 0.7841 & 0.1083 & 0.6205 \\
\hline$N=16$ & 0.7918 & 0.0619 & 0.8046 & 0.7928 & 0.0687 & 0.9579 \\
\hline$N=32$ & 0.7961 & 0.0432 & 0.8009 & 0.7955 & 0.0452 & -0.9741 \\
\hline$N=64$ & 0.7981 & 0.0303 & 0.8012 & 0.7983 & 0.0309 & 0.4419 \\
\hline \multicolumn{7}{|c|}{$p=256, q=1, V_{\text {rel }}(\mathbf{P})=0.8$} \\
\hline$N=8$ & 0.7831 & 0.0890 & 0.8098 & 0.7857 & 0.1056 & 1.7052 \\
\hline$N=16$ & 0.7918 & 0.0614 & 0.8031 & 0.7915 & 0.0681 & -0.3408 \\
\hline$N=32$ & 0.7961 & 0.0429 & 0.8022 & 0.7963 & 0.0460 & 0.3738 \\
\hline$N=64$ & 0.7981 & 0.0301 & 0.8004 & 0.7977 & 0.0305 & -0.9565 \\
\hline \multicolumn{7}{|c|}{$p=1024, q=1, V_{\text {rel }}(\mathbf{P})=0.8$} \\
\hline$N=8$ & 0.7831 & 0.0888 & 0.8088 & 0.7830 & 0.1074 & -0.0831 \\
\hline$N=16$ & 0.7918 & 0.0613 & 0.8057 & 0.7930 & 0.0683 & 1.2443 \\
\hline$N=32$ & 0.7961 & 0.0428 & 0.8014 & 0.7954 & 0.0454 & -0.9758 \\
\hline$N=64$ & 0.7981 & 0.0301 & 0.8006 & 0.7981 & 0.0308 & 0.0710 \\
\hline
\end{tabular}




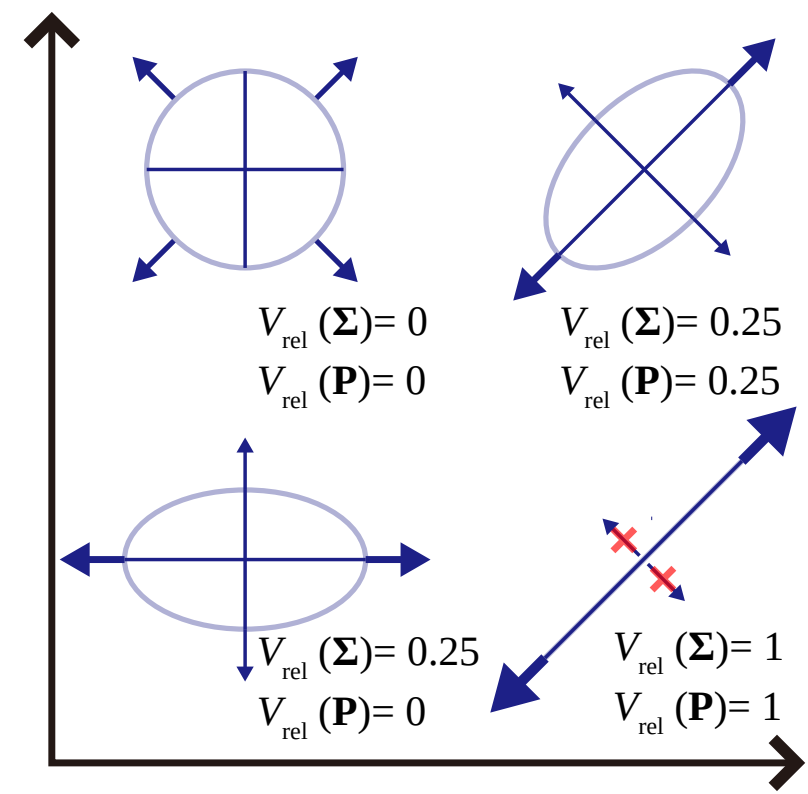

Figure 1. Schematic illustration of eigenvalue dispersion indices in bivariate cases. Ellipses representing equiprobability contours are shown on a Cartesian space of two hypothetical variables for four conditions, as well as the relative eigenvalue variance of the corresponding covariance and correlation matrices $\left(V_{\text {rel }}(\boldsymbol{\Sigma})\right.$ and $V_{\text {rel }}(\mathbf{P})$, respectively). The scale is arbitrary but identical for the two axes. The axes of each ellipse are proportional to square roots of the two eigenvalues of the respective covariance matrix. $V_{\text {rel }}(\Sigma)$ represents eccentricity of variation and is sensitive to differing scale changes between axes but not to rotation (change of eigenvectors), whereas $V_{\text {rel }}(\mathbf{P})$ represents magnitude of correlation and is insensitive to scale changes. Arrows schematically represent variation along major axes (whose directions are arbitrary when $\left.V_{\text {rel }}(\Sigma)=0\right)$. 
bioRxiv preprint doi: https://doi.org/10.1101/2021.06.19.449119; this version posted August 7, 2021. The copyright holder for this preprint (which was not certified by peer review) is the author/funder, who has granted bioRxiv a license to display the preprint in perpetuity. It is made available under aCC-BY-NC-ND 4.0 International license.
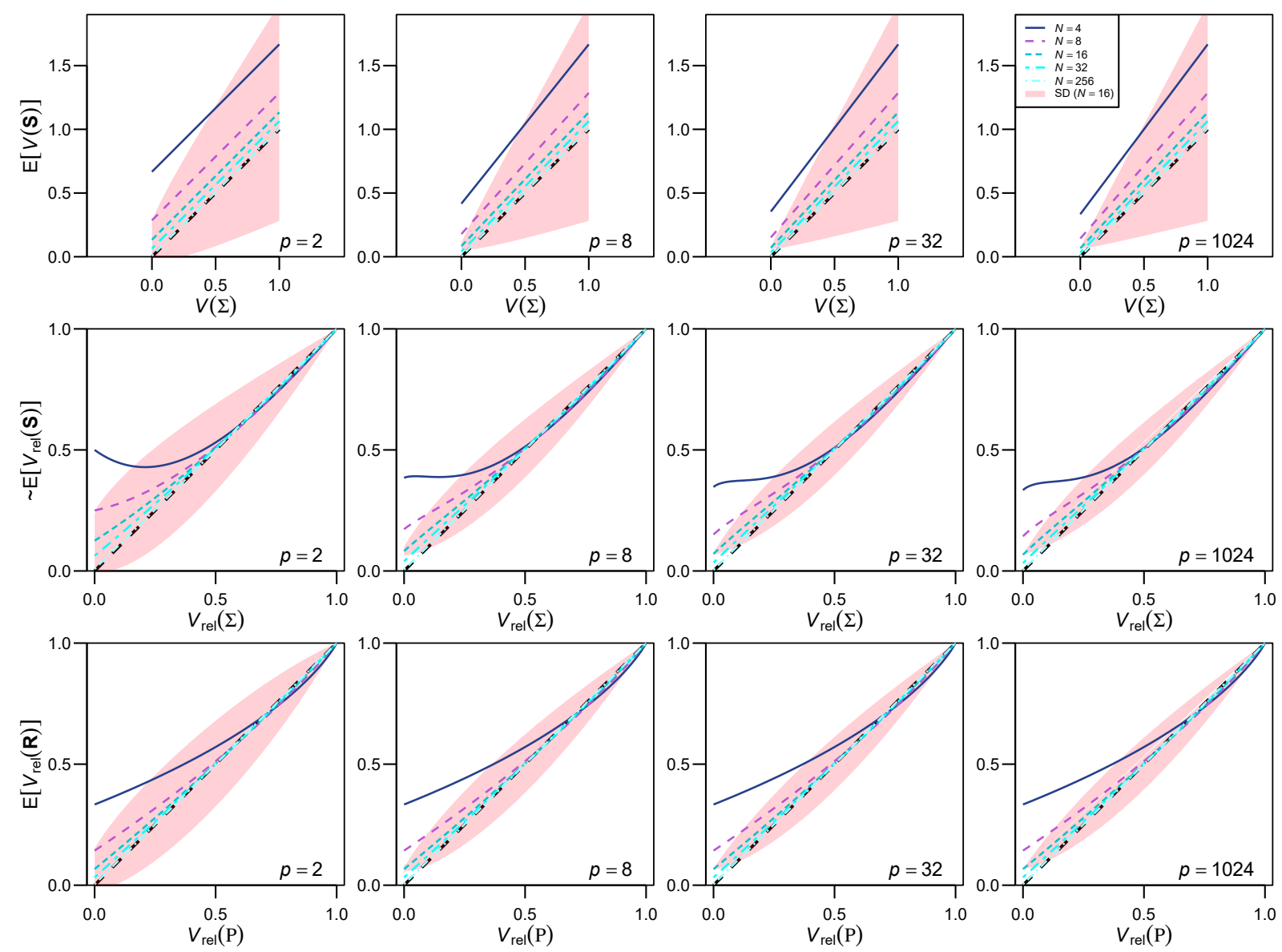

Figure 2. Profiles of the expectations of eigenvalue dispersion measures in selected conditions. The expectations of $V(\mathbf{S})$ (top row), $V_{\text {rel }}(\mathbf{S})$ (approximate; middle row), and $V_{\text {rel }}(\mathbf{R})$ (bottom row) are drawn with solid lines, for $p=2,8,32$, and 1024 (from left to right) and for $N=4,8,16,32$, and 256 (from top to bottom on the left end of each box). In all cases, $n=N-1$. The breadth of one standard deviation at $N$ $=16$ is also shown around the mean profiles with pink fills; these are approximations for $V_{\text {rel }}(\mathbf{S})$ and for $V_{\text {rel }}(\mathbf{R})$ with $p>2$ (the latter is from eq. 39; eqs. 36-38 yielded similar values under these conditions). Note that actual distributions might be skewed unlike these fills. There are generally many suites of eigenvalues corresponding to a single value of $V_{\text {rel }}$ and $\mathrm{E}\left[V_{\text {rel }}(\mathbf{R})\right]$ can also depend on eigenvector configurations; the profiles shown here are from such eigenvalue configurations that there is one large eigenvalue, with the rest being equally small, in which case $\mathrm{E}\left[V_{\text {rel }}(\mathbf{R})\right]$ does not depend on eigenvector configurations. The population covariance matrix $\boldsymbol{\Sigma}$ is scaled so that $\operatorname{tr} \boldsymbol{\Sigma}=p(p-1)^{-1 / 2}$. The initial decrease of the $\mathrm{E}\left[V_{\mathrm{rel}}(\mathbf{S})\right]$ profiles in some cases seems to be an artifact of approximation. See text for further technical details. 
bioRxiv preprint doi: https://doi.org/10.1101/2021.06.19.449119; this version posted August 7, 2021. The copyright holder for this preprint (which was not certified by peer review) is the author/funder, who has granted bioRxiv a license to display the preprint in perpetuity. It is made available under aCC-BY-NC-ND 4.0 International license.

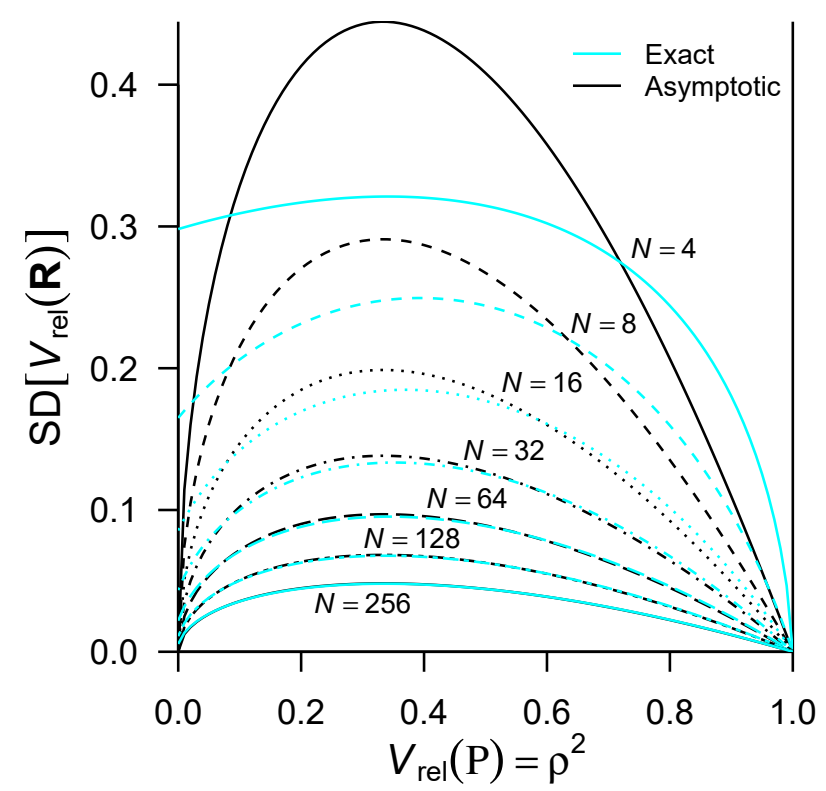

Figure 3. Comparison of exact and asymptotic standard deviations of $V_{\text {rel }}(\mathbf{R})$. Profiles of the exact (cyan lines; eq. 36) and asymptotic (black lines; eq. 39) standard deviations for $p=2$ are shown across the entire range of the population value $V_{\text {rel }}(\mathbf{P})$, for $N=4,8,16,32,64,128$, and 256 (from top to bottom as labeled; shown with different line styles). Note that the asymptotic profiles converge to 0 when $V_{\text {rel }}(\mathbf{P})=0$. 

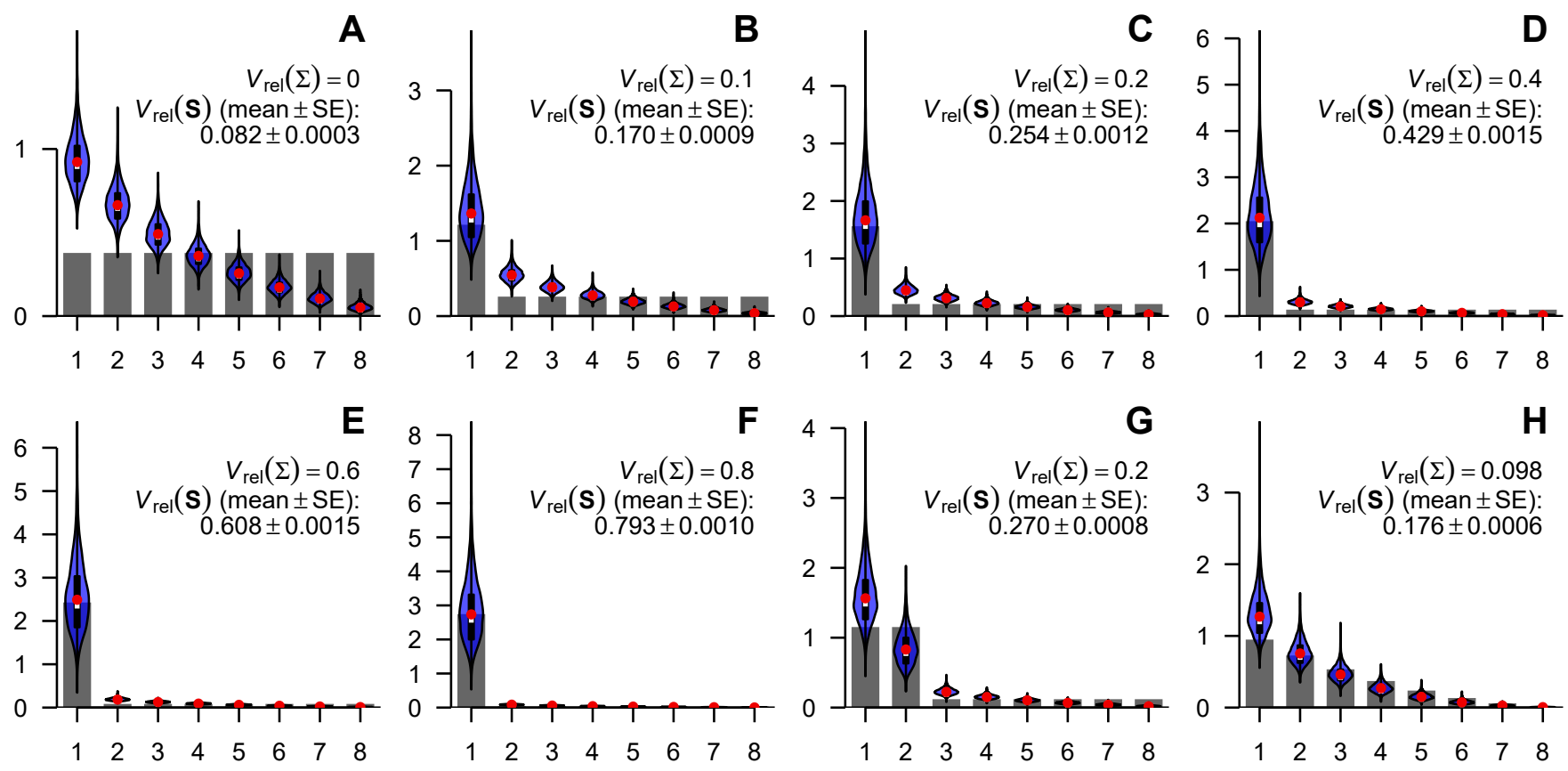

Figure 4. Selected population eigenvalue structures used in simulations and distributions of sample eigenvalues, examples for $p=8$. The eigenvalues of population covariance matrix are shown as scree plots, and distributions of sample eigenvalues with $N=16$ are shown as violin plots. A, null condition; B-G, $q$-large $\lambda$ conditions, $q=1(\mathbf{B}-\mathbf{F})$ or $2(\mathbf{G})$, with $V_{\text {rel }}(\boldsymbol{\Sigma})=0.1,0.2,0.4,0.6,0.8$, and 0.2 , respectively; $\mathbf{H}$, quadratically decreasing $\lambda$ condition. Red dots denote empirical means of sample eigenvalues, whereas white bars (mostly overlapping with red dots) denote medians. Thick black bars within violins denote interquartile ranges. Note different scales of vertical axes. 
bioRxiv preprint doi: https://doi.org/10.1101/2021.06.19.449119; this version posted August 7, 2021. The copyright holder for this preprint (which was not certified by peer review) is the author/funder, who has granted bioRxiv a license to display the preprint in perpetuity. It is made available under aCC-BY-NC-ND 4.0 International license.
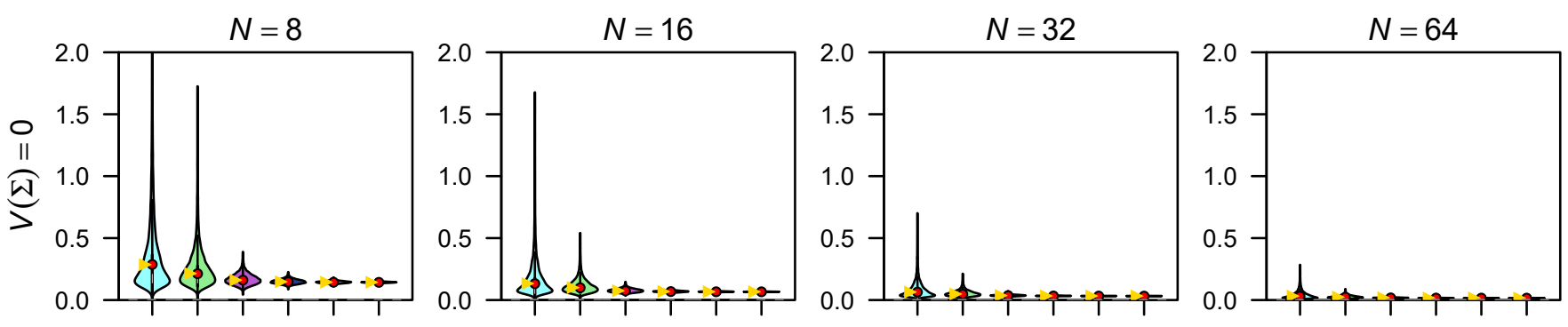

$p=\begin{array}{llllll}2 & 4 & 16 & 64 & 256 & 1024\end{array}$
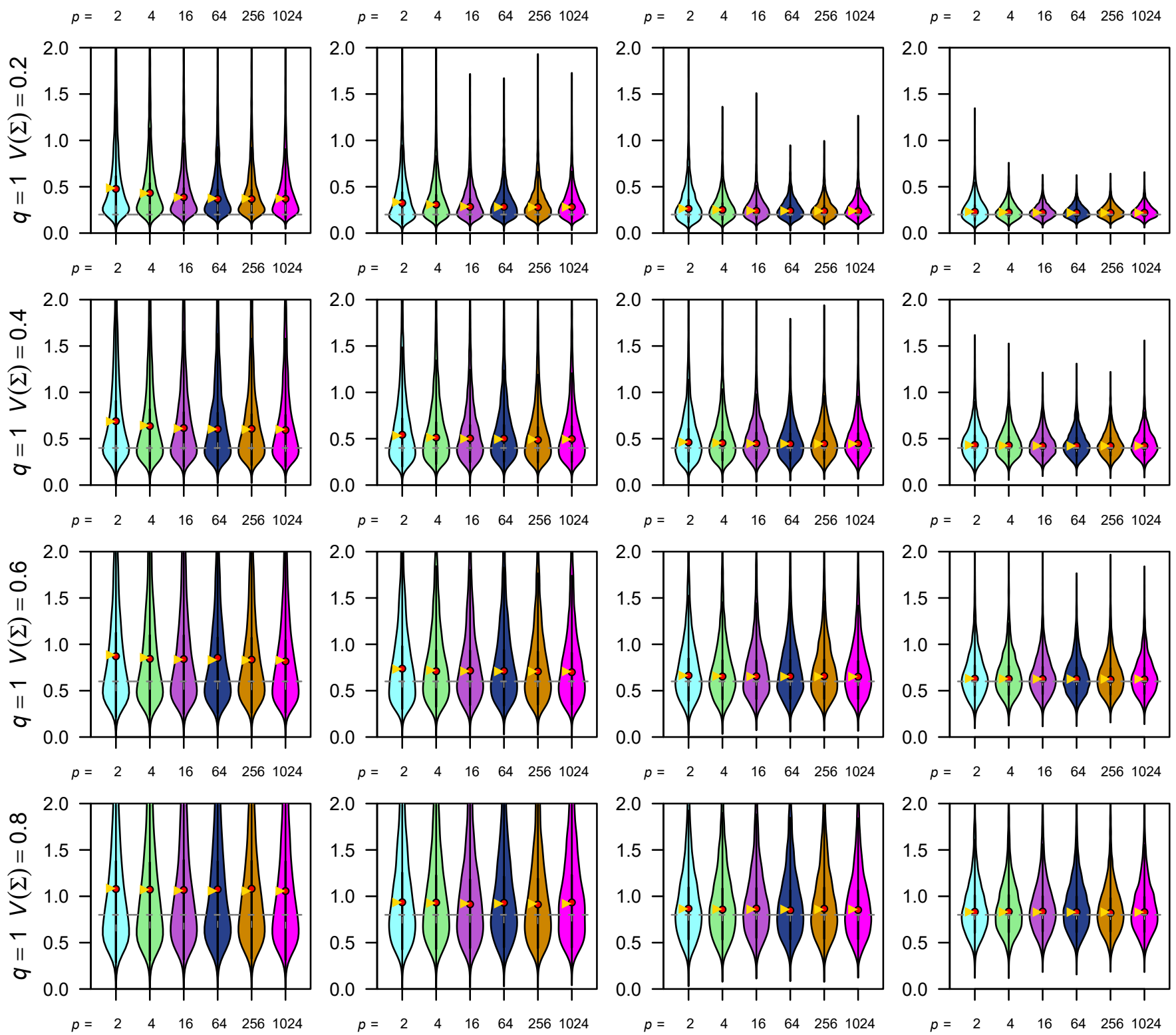

Figure 5. Selected simulation results for the eigenvalue variance of covariance matrix $V(\mathbf{S})$. Empirical distributions of simulated $V(\mathbf{S})$ values are shown as violin plots, whose tails extend to the extreme values. Red dots denote empirical means, whereas yellow triangles denote expectations (which are exact). Thick black bars within violins denote interquartile ranges, with white bars near the center (in some cases overlapping with red dots) denote medians. Rows of panels correspond to varying population values of $V(\boldsymbol{\Sigma})$ (under 1-large $\lambda$ conditions), whereas columns correspond to varying sample size $N$. Columns within each panel correspond to varying number of variables $p$. Note that extreme values in some panels are cropped for visual clarity. See Figure S4-S6 for full results. 
bioRxiv preprint doi: https://doi.org/10.1101/2021.06.19.449119; this version posted August 7, 2021. The copyright holder for this preprint (which was not certified by peer review) is the author/funder, who has granted bioRxiv a license to display the preprint in perpetuity. It is made available under aCC-BY-NC-ND 4.0 International license.
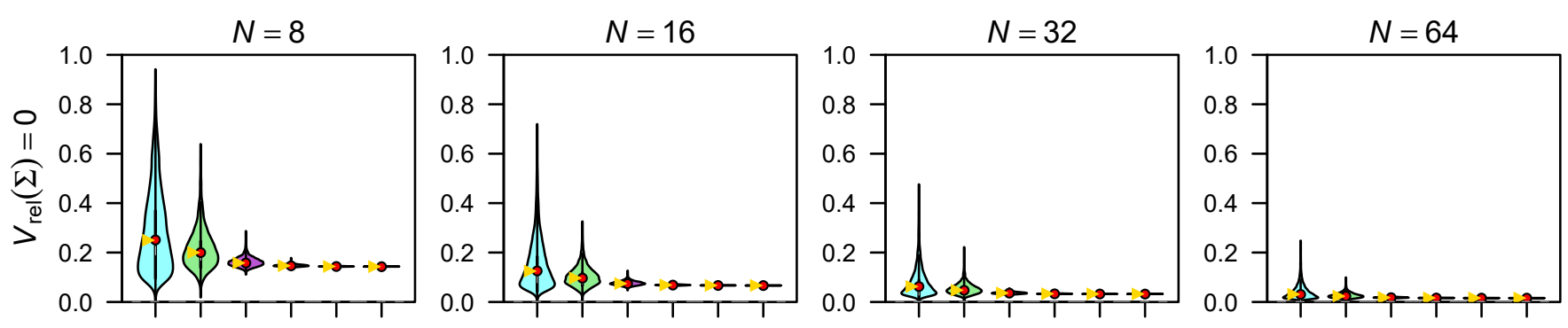

$p=\begin{array}{llllll}2 & 4 & 16 & 64 & 256 & 1024\end{array}$
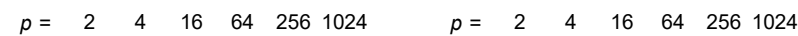

$p=\begin{array}{llllll}2 & 4 & 16 & 64 & 256 & 1024\end{array}$
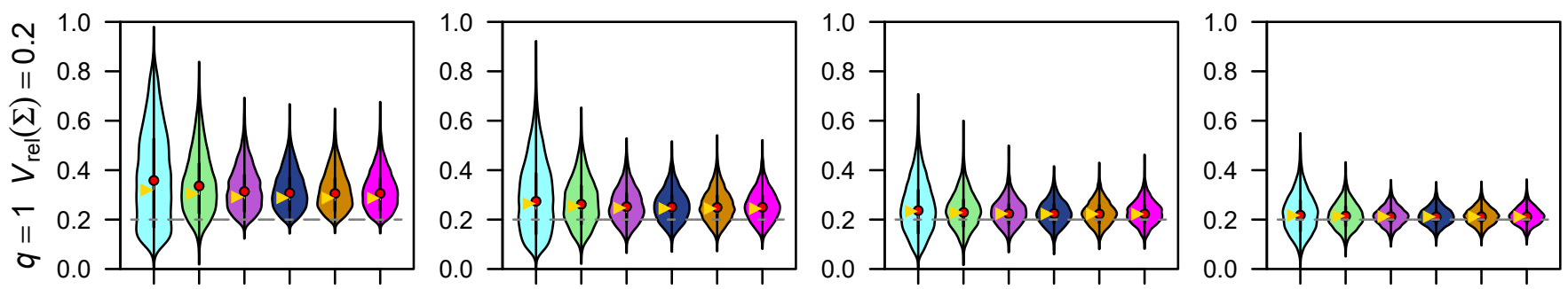

$p=\begin{array}{llllll}2 & 4 & 16 & 64 & 256 & 1024\end{array}$
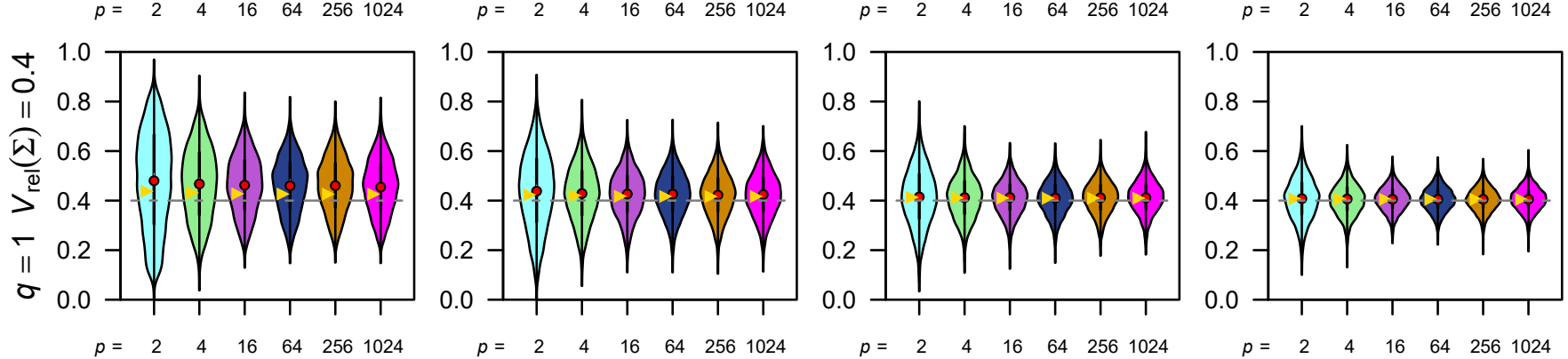

$p=2 \quad 2 \quad 4 \quad 16 \quad 64 \quad 256 \quad 1024$
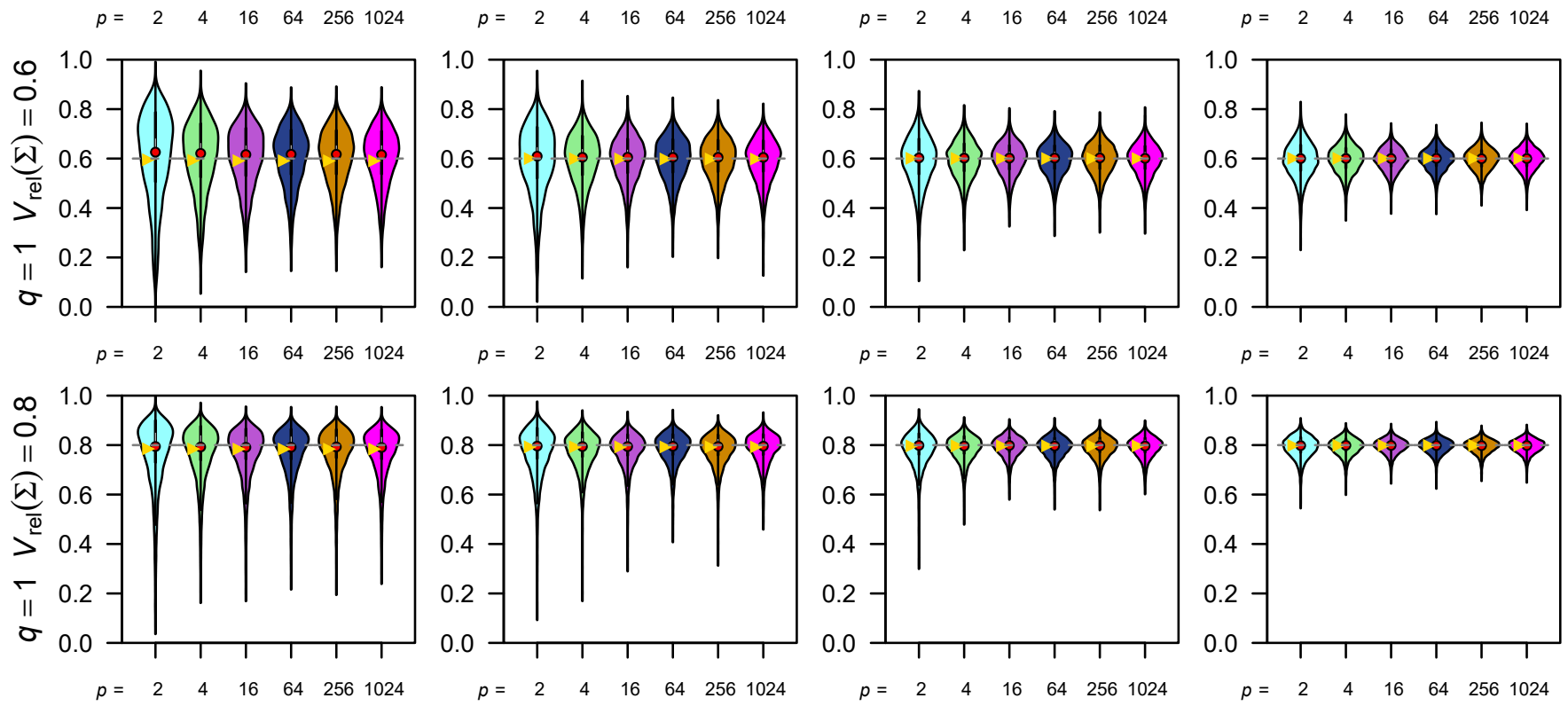

Figure 6. Selected simulation results for the relative eigenvalue variance of covariance matrix $V_{\text {rel }}(\mathbf{S})$. Empirical distributions of simulated $V_{\text {rel }}(\mathbf{S})$ values are shown as violin plots. Yellow triangles denote expectations (which are approximate except under the null condition). Rows of panels correspond to varying population values of $V_{\text {rel }}(\boldsymbol{\Sigma})$ (under 1-large $\lambda$ conditions). Other legends are as in Fig. 5. See Figure S6-S8 for full results. 
bioRxiv preprint doi: https://doi.org/10.1101/2021.06.19.449119; this version posted August 7, 2021. The copyright holder for this preprint (which was not certified by peer review) is the author/funder, who has granted bioRxiv a license to display the preprint in perpetuity. It is made available under aCC-BY-NC-ND 4.0 International license.
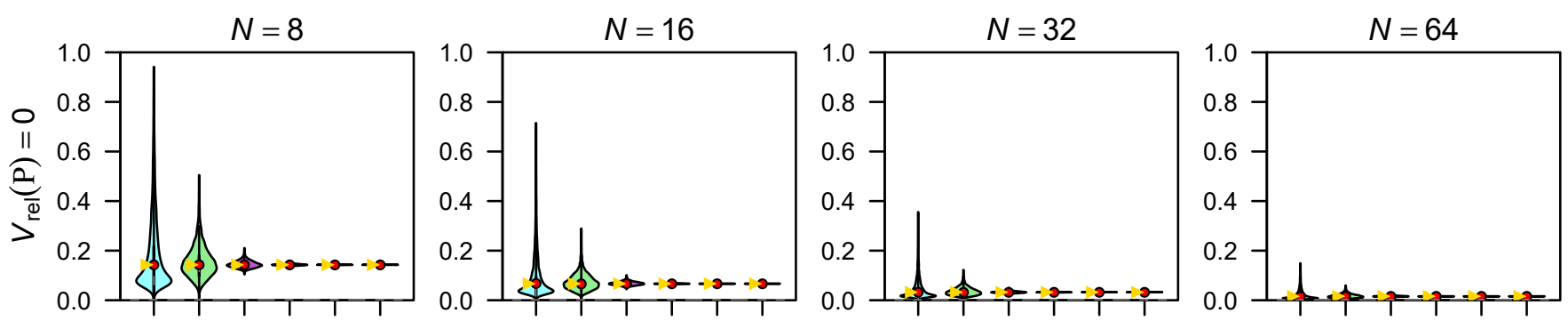

$p=\begin{array}{llllll}2 & 4 & 16 & 64 & 256 & 1024\end{array}$
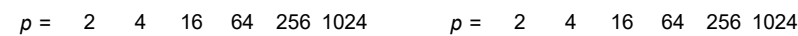

$p=\begin{array}{llllll}2 & 4 & 16 & 64 & 256 & 1024\end{array}$
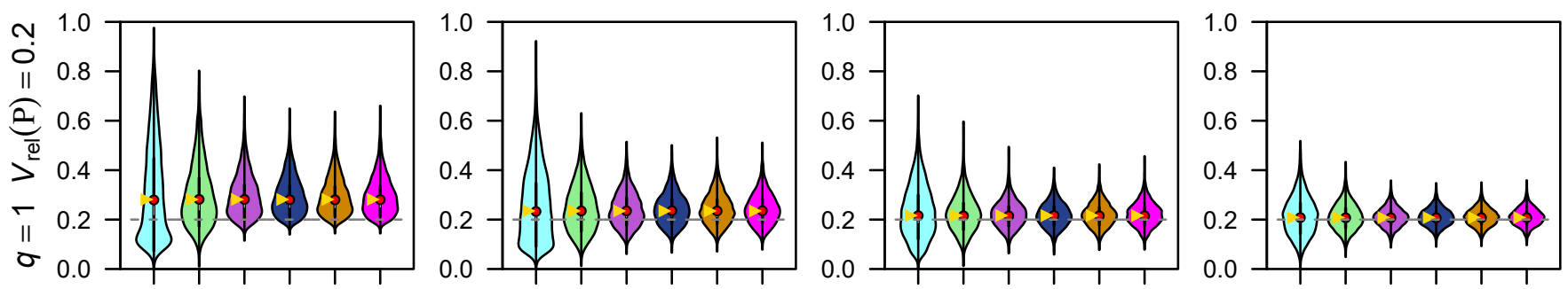

$p=\begin{array}{llllll}2 & 4 & 16 & 64 & 256 & 1024\end{array}$
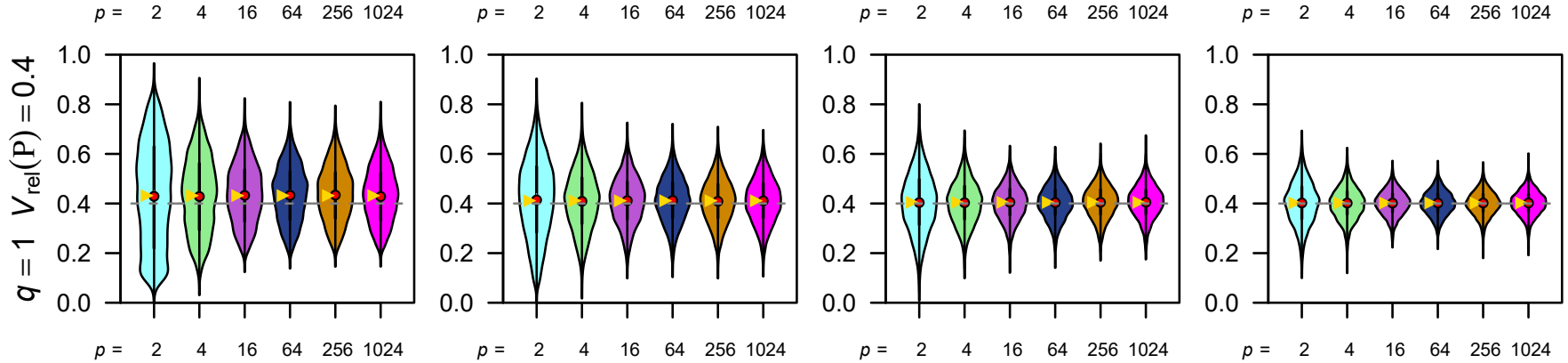

$p=2 \quad 2 \quad 4 \quad 16 \quad 64 \quad 256 \quad 1024$
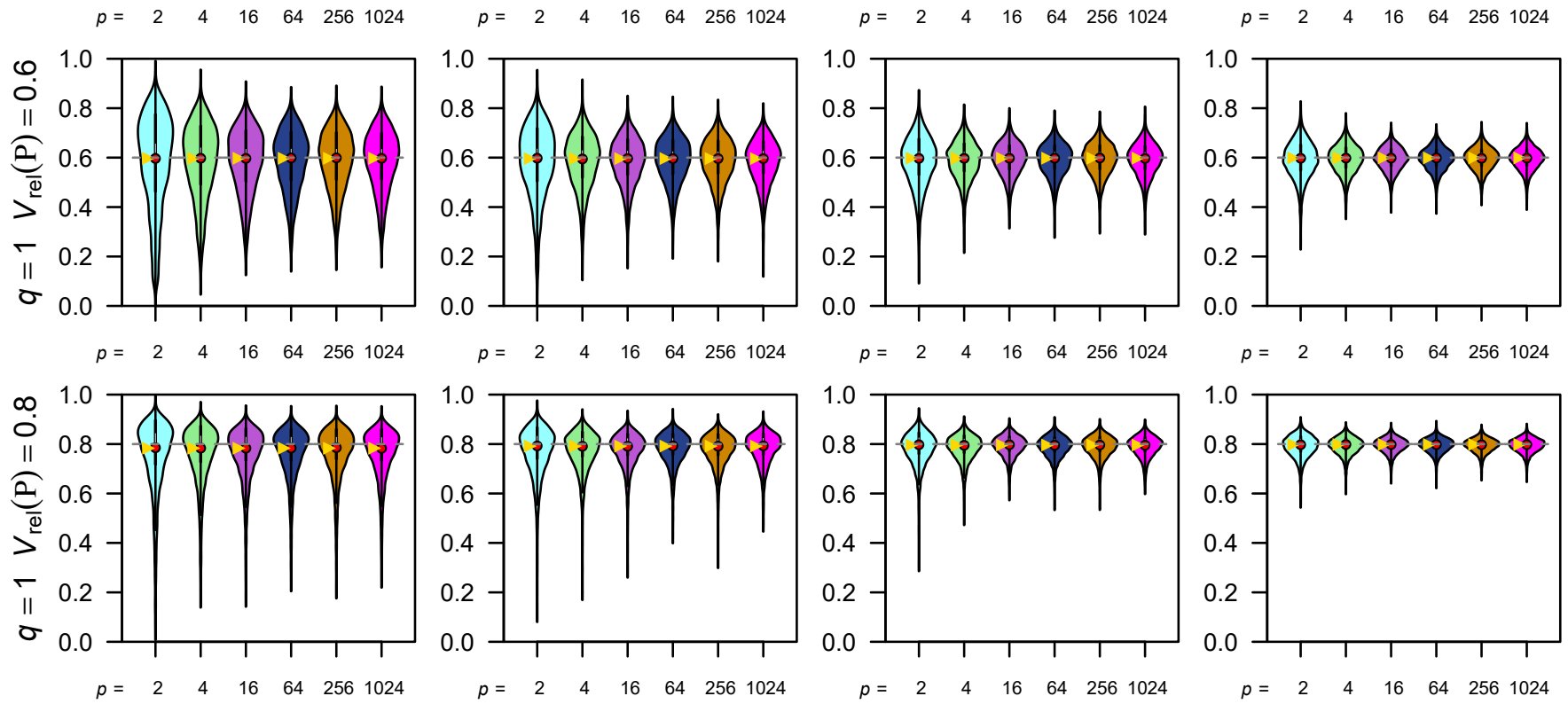

Figure 7. Selected simulation results for the relative eigenvalue variance of correlation matrix $V_{\text {rel }}(\mathbf{R})$. Empirical distributions of simulated $V_{\text {rel }}(\mathbf{R})$ values are shown as violin plots. Yellow triangles denote expectations (which are exact). Rows of panels correspond to varying population values of $V_{\text {rel }}(\mathbf{P})$ (under 1-large $\lambda$ conditions). Other legends are as in Fig. 5. See Figure S6, S9, and S10 for full results. 